\title{
DUALITY FOR CLASSICAL $p$-ADIC GROUPS: THE HALF-INTEGRAL CASE
}

\author{
CHRIS JANTZEN
}

\begin{abstract}
Let $G$ be a classical $p$-adic group and let $\pi$ be a smooth irreducible representation of $G$. In this paper, we consider the problem of calculating the dual (in the sense of Aubert and Schneider-Stuhler) $\hat{\pi}$. More precisely, if $\pi$ is specified by its Langlands data, the problem is to determine the Langlands data for $\hat{\pi}$. This problem reduces (based on supercuspidal support) to two main cases: half-integral reducibility and integral reducibility; the latter is addressed here.
\end{abstract}

\section{INTRODUCTION}

We begin by recalling the definition of the duality operator (see $[\mathrm{Au}], \mathrm{S}-\mathrm{S}]$ ). Let $F$ be a $p$-adic field and let $G$ be the $F$-points of a connected reductive group defined over $F$. If $\Pi$ denotes the set of simple roots for $G$ and $\pi$ is a smooth, finite length representation of $G$, the dual $D_{G}(\pi)$ is defined as

$$
D_{G}(\pi)=\sum_{\Phi \subset \Pi}(-1)^{|\Phi|} i_{G, M_{\Phi}} \circ r_{M_{\Phi}, G}(\pi)
$$

$\left(i_{G, M_{\Phi}}, r_{M_{\Phi}, G}\right.$ denoting normalized parabolic induction and Jacquet module - see [B-Z or the next section), with the sum taking place in the Grothendieck group (with a similar definition for the nonconnected group $O(2 n, F)$;cf. [J4]). The duality operator is an involution which takes irreducible representations to irreducible representations (up to \pm ), and behaves well with respect to the parabolic induction and Jacquet module functors (cf. Théoreme 1.1 of $\mathrm{Au}$. for more precise statements). We write $\hat{\pi}$ for $\pm D_{G}(\pi)$, whichever is nonnegative.

Historically, such duality operators were first defined for finite groups of Lietype by Curtis [Cur, Alvis [Alv, and Kawanaka Kaw; Deligne-Lusztig proved that irreducibility is preserved in [D-L1], [D-L2]. For Hecke algebras, the IwahoriMatsumoto involution [I-M] is older, but the action on representations results from an involution on the Hecke algebra; the characterization (1.1) did not appear until later in Kat. Similarly, the Zelevinsky involution for $p$-adic general linear groups [Ze] is a special case of duality, but defined somewhat differently; it was conjectured to preserve irreducibility. The general version for connected reductive $p$-adic groups was done by Aubert $[\mathrm{Au}]$ and Schneider-Stuhler $[\mathrm{S}-\mathrm{S}]$; they also established the connection between the Zelevinsky involution and duality. There is also a more recent interpretation in terms of Arthur packets. There is an obvious involution on Arthur packets corresponding to the interchange of the two copies of $S L(2, \mathbb{C})$ in

Received by the editors January 15, 2018, and, in revised form, July 19, 2018.

2010 Mathematics Subject Classification. Primary 22 E50.

This research was supported in part by NSA grant H98230-13-1-0237. 
the parameters; this also turns out to be given by duality (see section 7.1 of [Art, Mœ3.).

The problem considered here is that of providing an algorithm for calculating $D_{G}$ for representations of classical groups. More precisely, if $\pi$ is specified by its Langlands data, the problem is to determine the Langlands data for $\hat{\pi}$. In the case of general linear groups, such an algorithm was originally given in [M-W. Later, a more explicit formula was given in $[\mathrm{K}-\mathrm{Z}]$. An algorithm, more along the lines of [M-W], was given as an application of some results in [J5]; the algorithm below uses the same underlying strategy.

Beyond general interest and the considerations above, there are a couple of additional reasons for our interest in this problem. First, the duality operator can be useful in analyzing induced representations - e.g., techniques may apply to the dual induced representation which do not apply to the original. Conclusions may be drawn on the dual side, then transferred back (e.g., [J1], [B-J2]). Also, the IwahoriMatsumoto involution is known to preserve unitarity ([B-M]$)$; more general duality has also found a role in the determination of the unitary dual (e.g., [Mu1, [Mu-T]).

By [J2] (and [J4]), the problem of determining $\hat{\pi}$ in general may be reduced to the problem of determining $\hat{\pi}$ when $\pi \in R((\rho, \beta) ; \sigma)$, i.e., when $\pi$ has supercuspidal support on $\left\{\nu^{x} \rho\right\}_{x \in \beta+\mathbb{Z}} \cup\{\sigma\}, 0 \leq \beta \leq \frac{1}{2}$ if $\rho \cong \check{\rho}$ (resp., $\left\{\nu^{x} \rho, \nu^{-x} \check{\rho}\right\}_{x \in \beta+\mathbb{Z}} \cup\{\sigma\}$, $0 \leq \beta<1$ if $\rho \neq \check{\rho}$ )); here $\rho$ denotes an irreducible unitary supercuspidal representation for a general linear group, $\sigma$ an irreducible supercuspidal representation of a classical group, and $\check{\rho}$ the contragredient (in the symplectic or orthogonal cases) or its conjugate (in the unitary case) - see section 2.1. Now, the induced representation $i_{G, M}\left(\nu^{x} \rho \otimes \sigma\right)$ is reducible for some (exactly one) $x \geq 0$ if and only if $\check{\rho} \cong \rho$; we let $\operatorname{red}(\rho ; \sigma)$ denote this value. This paper addresses the case $\operatorname{red}(\rho ; \sigma) \equiv \frac{1}{2}$ $\bmod 1$. The other possibility when $\rho \cong \check{\rho}$-i.e., $\operatorname{red}(\rho ; \sigma) \in \mathbb{Z}$ - has certain technical difficulties discussed later. However, many of the results established along the way hold in general.

Before describing the algorithm itself, we first need to recall a key definition. Let $G_{n}(F)$ be from one of the families of classical groups under consideration (symplectic, odd special orthogonal, even orthogonal, unitary-see section 2). For $1 \leq m \leq n$, we have a (maximal proper) standard parabolic subgroup with Levi factor $M_{(m)} \cong G L(m, F) \times G_{n-m}(F)$. For $\pi$ a representation of $G$, set

$$
\mu^{*}(\pi)=\sum_{i=1}^{n} r_{M_{(i)}, G}(\pi),
$$

with the sum in $R \otimes R[S]$ - see section 2.1. This was originally defined in [T1], and has many useful properties, discussed in more detail in the next section. However, the definition is sufficient for the purposes at hand. In particular, it enables us to define the following (Definition 3.1.1 of [J7]).

Definition 1.1. For $X$ a set of (not necessarily unitary) supercuspidal representations of general linear groups, let $f=f_{\pi}(X)$ be the largest value such that a (minimal nonzero) Jacquet module of $\pi$ has a term of the form $\nu^{x_{1}} \rho_{1} \otimes \cdots \otimes \nu^{x_{f}} \rho_{f} \otimes \ldots$ with $\nu^{x_{1}} \rho_{1}, \ldots, \nu^{x_{f}} \rho_{f} \in X$ (where $\nu=|\operatorname{det}|$ as in [Z] $)$. We let

$$
\mu_{X}^{*}(\pi)=\sum_{i} \lambda_{i} \otimes \theta_{i}
$$


where the sum is over all irreducible $\lambda_{i} \otimes \theta_{i} \leq \mu^{*}(\pi)$ for which a (minimal nonzero) Jacquet module of $\lambda_{i}$ contains a term of the form $\nu^{x_{1}} \rho_{1} \otimes \cdots \otimes \nu^{x_{f}} \rho_{f}$, with $\nu^{x_{1}} \rho_{1}, \ldots, \nu^{x_{f}} \rho_{f} \in X$.

Suppose $X$ has the property that $\nu^{x} \rho \in X \Rightarrow \nu^{-x} \check{\rho} \notin X$. If $\pi$ is irreducible, we have the following key properties (section 3.1 of [J7]):

- $\mu^{*}(\pi)$ consists of a single representation, denoted $\lambda_{\pi}(X) \otimes \theta_{\pi}(X)$.

- $\pi \hookrightarrow i_{G, M}\left(\lambda_{\pi}(X) \otimes \theta_{\pi}(X)\right)$ as unique irreducible subrepresentation.

-If $\mu_{X}^{*}\left(\pi_{1}\right)=\mu_{X}^{*}\left(\pi_{2}\right)$, then $\pi_{1} \cong \pi_{2}$ (follows from above).

Note that we have $|X|=1$ for most applications.

We remark that if $\sigma$ is a supercuspidal representation of one of the classical groups under consideration, then the induced representation $i_{G, M}\left(\nu^{x} \rho \otimes \sigma\right)$ is reducible for some $x \in \mathbb{R}$ if and only if $\check{\rho} \cong \rho$, making this the most interesting case. However, if $\check{\rho} \cong \rho$, then one cannot have $\nu^{x} \rho \in X \Rightarrow \nu^{-x} \check{\rho} \notin X$ when $x=0$. As a consequence, the case $x=0$ is avoided in the algorithm below. Section 7 contains more on the issues which arise in this case.

We now turn to the algorithm for duality. Assuming an algorithm for calculating $\mu_{X}^{*}(\pi)$, we may calculate $\hat{\pi}$ as follows.

Algorithm (Algorithm for computing $\hat{\pi}$ ).

(1) Calculate $\mu_{\left\{\nu^{x} \rho\right\}}^{*}(\pi)=\left(\nu^{x} \rho\right)^{f_{\pi}\left(\nu^{x} \rho\right)} \otimes \theta_{\pi}\left(\nu^{x} \rho\right)$ for some $\nu^{x} \rho, x \neq 0$, having $f_{\pi}\left(\nu^{x} \rho\right)>0$ (i.e., $\mu_{X}^{*}(\pi)$ for $\left.X=\left\{\nu^{x} \rho\right\}\right)$. This may be done using the algorithm described in section 3 .

(2) By properties of duality, it follows that $\mu_{\left\{\nu^{-x} \breve{\rho}\right\}}^{*}(\hat{\pi})=\left(\nu^{-x} \check{\rho}\right)^{f_{\pi}\left(\nu^{x} \rho\right)} \otimes \widehat{\theta_{\pi}\left(\nu^{x} \rho\right)}$. Note that as $\theta_{\pi}\left(\nu^{x} \rho\right)$ is from a group of lower rank, we may inductively assume that it has been calculated. In practice, we work iteratively, treating the algorithm as reducing the rank.

(3) Recover $\hat{\pi}$ from $\mu_{\left\{\nu^{-x} \breve{\rho}\right\}}^{*}(\hat{\pi})=\left(\nu^{-x} \check{\rho}\right)^{f_{\pi}\left(\nu^{x} \rho\right)} \otimes \widehat{\theta_{\pi}\left(\nu^{x} \rho\right)}$. Note that by the properties of $\mu_{X}^{*}$ above, $\mu_{\left\{\nu^{-x} \breve{\rho}\right\}}^{*}(\hat{\pi})$ suffices to determine $\hat{\pi}$. (There are only a finite number of possibilities for $\hat{\pi}$ having the correct supercuspidal support. In principle, one could calculate $\mu_{\left\{\nu^{-x} \breve{\rho}\right\}}^{*}$ for all of them; in practice, only a small number of these need to be checked.)

The above algorithm is demonstrated in Example 1 of section 4.1, where $\hat{\pi}$ is computed in three iterations.

The key in implementing the algorithm above is the ability to calculate $\mu_{\left\{\nu^{x} \rho\right\}}^{*}(\pi)$; the construction of an algorithm for doing so constitutes the bulk of this paper. We now take a moment to discuss this.

Consider $X=\left\{\nu^{x} \rho\right\}$, with $\rho$ irreducible unitary supercuspidal. Write $\pi=$ $L(\Delta ; T)$ in the subrepresentation setting of the Langlands classification (see section 2.1). We have

$$
\pi \hookrightarrow i_{G, M}(\mathcal{L}(\Delta) \otimes T)
$$

using $\mathcal{L}(\Delta)$ to denote the representation of the appropriate general linear group having Langlands data $\Delta$ (also section 2.1). Intuitively, copies of $\nu^{x} \rho$ can come from one of three sources:

(1) terms $\nu^{x} \rho \otimes \ldots$ in $r_{M^{\prime}, G^{\prime}}(\mathcal{L}(\Delta))$,

(2) terms $\cdots \otimes \nu^{-x} \check{\rho}$ in $r_{M^{\prime \prime}, G^{\prime}}(\mathcal{L}(\Delta))$,

(3) terms $\nu^{x} \rho \otimes \ldots$ in $r_{L, G^{\prime \prime}}(T)$. 
The analysis has three parts. First, the case of $\pi=T$ (tempered) must be addressed. In this case, the result is done in [J8], based on the extended MoglinTadic data for $T$, and is summarized in Theorem 3.1. Note that this is essentially a formula rather than an algorithm.

The second case is $x<0$ (relevant only for nontempered $\pi$ ). This case is relatively easy as only terms of the form $\nu^{x} \rho \otimes \ldots$ in $r_{M^{\prime}, G^{\prime}}(\mathcal{L}(\Delta))((1)$ above) can contribute. In this case, one calculates $m_{\left\{\nu^{x} \rho\right\}}^{*}(\mathcal{L}(\Delta))$ - the analogue to $\mu_{\left\{\nu^{x} \rho\right\}}^{*}$ for general linear groups (see section 2.1) - which is known (Theorem 2.2.1 of [J5]). Then,

$$
\begin{gathered}
m_{\left\{\nu^{x} \rho\right\}}^{*} \mathcal{L}(\Delta)=\left(\nu^{x} \rho\right)^{f} \otimes \mathcal{L}\left(\Delta^{\prime}\right) \\
\Downarrow \\
\mu_{\left\{\nu^{x} \rho\right\}}^{*}(\pi)=\left(\nu^{x} \rho\right)^{f} \otimes L\left(\Delta^{\prime} ; T\right) .
\end{gathered}
$$

The third case is $x>0$ with $\pi$ nontempered. In this case, there are two parts to the algorithm. The first resolves the problem assuming one can determine $\mu_{\left\{\nu^{x} \rho\right\}}^{*}$ for representations of the form $L\left(\left(\nu^{-x} \rho\right)^{k}, \delta\left(\left[\nu^{-x} \rho, \nu^{x-1} \rho\right]\right)^{\ell} ; T\right)$; the second addresses this special case.

The arguments in this paper are ultimately built from the Møglin-Tadic classification of discrete series ([M-T $]$ ) and the machinery needed for that classification. Their work is done under the assumption that their Basic Assumption (BA) holds. Note that in the quasi-split case, when $\operatorname{char}(F)=0,(\mathrm{BA})$ follows from Art] (see Mœ4]), so their results hold unconditionally. When $\operatorname{char}(F)>0$ - still in the quasisplit case - (BA) is proven in G-L, contigent on the Working Hypothesis of [G-L. In those cases where it is not already established, we assume (BA); our results then hold in the generality of [M-T], that is, to the same classical groups considered there.

We close by briefly describing the contents of this paper. In the next section, we introduce notation and give some background results. In section 3 , we give the algorithm for calculating $\mu_{\left\{\nu^{x} \rho\right\}}^{*}$, breaking the presentation into several subsections depending on the value of $x$ and the representation under consideration. Note that only the last of these subsections uses $\operatorname{red}(\rho ; \sigma) \equiv \frac{1}{2} \bmod 1$, and also assumes $\pi \in R\left(\left(\rho, \frac{1}{2}\right) ; \sigma\right)$ (the most interesting value of $\beta$ in this situation). To streamline the presentation, proofs of the results in section 3 are deferred until later in the paper. In section 4, we give an example to illustrate the algorithms in action. The proofs of the main results follow: sections 5 , 6 contain the proofs of the results from section 3 . In section 7 we make some remarks on what breaks down when $x=0$. In section 8 , we retain the assumption $\operatorname{red}(\rho ; \sigma) \equiv \frac{1}{2} \bmod 1$, and take up what happens when $\beta \neq \frac{1}{2}$.

\section{Notation AND PRELIMINARIES}

2.1. Notation and preliminaries. Let $F$ be a $p$-adic field. We make no restrictions on $\operatorname{char}(F)$, but remind the reader that the status of the Møglin-Tadic classification depends (in part) on the characteristic of $F$. We consider the same families of (not necessarily quasi-split) classical groups as in [M-T]: symplectic, odd special orthogonal, even special orthogonal (nonsplit only), even orthogonal (split only), and even and odd unitary groups (with $F^{\prime} / F$ the associated separable quadratic extension). For a more detailed description, see section 1 of [M-T]. 
We now discuss some structure theory from [Ze] and [T1, [Ba]. First, let $S(n, F)$ denote the rank $n$ member of one of the families of classical groups under consideration and set

$$
R=\bigoplus_{n \geq 0} \mathcal{R}(G L(n, F)) \text { and } R[S]=\bigoplus_{n \geq 0} \mathcal{R}(S(n, F)),
$$

where $\mathcal{R}(G)$ denotes the Grothendieck group of the category of smooth finite length representations of $G$. We define multiplication on $R$ as follows: suppose $\rho_{1}, \rho_{2}$ are representations of $G L\left(n_{1}, F\right), G L\left(n_{2}, F\right)$, resp. We have $M=G L\left(n_{1}, F\right) \times$ $G L\left(n_{2}, F\right)$ the Levi factor of a standard parabolic subgroup of $G=G L(n, F)$, where $n=n_{1}+n_{2}$, and set $\tau_{1} \times \tau_{2}=i_{G, M}\left(\tau_{1} \otimes \tau_{2}\right)$ (normalized parabolic inductionsee $[\mathrm{B}-\mathrm{Z}$ ). This extends (after semisimplification) to give the multiplication $\times$ : $R \times R \longrightarrow R$. To describe the comultiplication on $R$, let $M_{(i)}$ denote the standard Levi factor for $G=G L(n, F)$ having $M_{(i)}=G L(i, F) \times G L(n-i, F)$. For a representation $\tau$ of $G L(n, F)$, we define

$$
m^{*}(\tau)=\sum_{i=0}^{n} r_{M_{(i)}, G}(\tau),
$$

the sum of semisimplified Jacquet modules (lying in $R \otimes R$ ). This extends to a map $m^{*}: R \longrightarrow R \otimes R$. We note that with this multiplication and comultiplication (and antipode map given by the Zelevinsky involution, a special case of the general duality operator of $[\mathrm{Au}],[\mathrm{S}-\mathrm{S}]), R$ is a Hopf algebra. There are two analogues for general linear groups of the $\mu_{X}^{*}$ discussed in the introduction: $m_{X}^{*}$ and ${ }_{X} m^{*}$. For an irreducible representation $\pi$, we let $f=f_{\pi}(X)$ (resp., $g=g_{\pi}(X)$ ) be the largest value such that a minimal nonzero Jacquet module of $\pi$ has a term of the form $\nu^{x_{1}} \rho_{1} \otimes \cdots \otimes \nu^{x_{f}} \rho_{f} \otimes \ldots$ (resp., of the form $\cdots \otimes \nu^{x_{g}} \rho_{g} \otimes \cdots \otimes \nu^{x_{1}} \rho_{1}$ ) with all $\nu^{x_{i}} \rho_{i} \in X, 1 \leq i \leq f$ (resp., $1 \leq i \leq g$ ). The analogue of (1.2) holds without restriction on $X$ (Lemma 2.1.2 of [J5]); we define $m_{X}^{*}$ and ${ }_{X} m^{*}$ accordingly.

Recall that for $a, b$ with $a \leq b$ and $b-a \in \mathbb{Z}, \delta\left(\left[\nu^{a} \rho, \nu^{b} \rho\right]\right)$ denotes the generalized Steinberg representation associated to the segment $\left[\nu^{a} \rho, \nu^{b} \rho\right]$, i.e., the unique irreducible subrepresentation of $\nu^{b} \rho \times \nu^{b-1} \rho \times \cdots \times \nu^{a} \rho$ ([Z] $)$. The unique irreducible subrepresentation of $\nu^{a} \rho \times \nu^{a+1} \rho \times \cdots \times \nu^{b} \rho$ is denoted $\zeta\left(\left[\nu^{a} \rho, \nu^{b} \rho\right]\right)$. Note that $\zeta\left(\left[\nu^{a} \rho, \nu^{b} \rho\right]\right)$ is dual to $\delta\left(\left[\nu^{a} \rho, \nu^{b} \rho\right]\right)$.

Next, suppose $\tau$ is a representation of $G L\left(n_{1}, F\right)$ and $\theta$ a representation of $S\left(n_{2}, F\right)$. We have $M=G L\left(n_{1}, F\right) \times S\left(n_{2}, F\right)$ the Levi factor of a standard parabolic subgroup of $G=S(n, F)$, with $n=n_{1}+n_{2}$, and set $\tau \rtimes \theta=i_{G, M}(\tau \otimes \theta)$. If one extends $\rtimes$ to a map $\rtimes: R \otimes R[S] \longrightarrow R[S]$, we have $R[S]$ as a module over $R$. To describe its comodule structure, let $M_{(i)}=G L(i, F) \otimes S(n-i, F)$, a standard Levi factor for $G=S(n, F)$. For a representation $\pi$ of $S(n, F)$, we define

$$
\mu^{*}(\pi)=\sum_{i=0}^{n} r_{M_{(i)}, G}(\pi),
$$

the sum of (normalized) semisimplified Jacquet modules (lying in $R \otimes R[S]$ ). This extends to a map $\mu^{*}: R[S] \longrightarrow R \otimes R[S]$. In addition to $\mu_{X}^{*}$ introduced earlier, there is another variant of this which is needed occasionally in what follows. For an irreducible representation $\lambda$ of a general linear group and a representation $\pi$ of one of the classical groups under consideration, we let $\mu_{\lambda}^{*}(\pi)$ be the sum of everything 
in $\mu^{*}(\pi)$ having first factor isomorphic to $\lambda$. More precisely, if $\mu^{*}(\pi)=\sum_{i} \lambda_{i} \otimes \xi_{i}$, we let $\mu_{\lambda}^{*}(\pi)=\sum_{i \in I_{\lambda}} \lambda_{i} \otimes \xi_{i}$, where $I_{\lambda}=\left\{i \mid \lambda_{i} \cong \lambda\right\}$.

For unitary groups, let $\xi$ denote the nontrivial element of the Galois group of the underlying quadratic extension. For a representation $\pi$ of $S(n, F)$, we then define

$$
\check{\pi}=\left\{\begin{array}{l}
\tilde{\pi} \circ \xi \text { for unitary groups } \\
\tilde{\pi} \text { otherwise }
\end{array}\right.
$$

where denotes contragredient. Using this, we may give $R[S]$ the structure of an $M^{*}$-module over $R$ ([T1], [Ba], [M-T] $)$ :

Theorem 2.1. Define $M^{*}: R \longrightarrow R \otimes R$ by

$$
M^{*}=(m \otimes 1) \circ\left({ }^{\sim} \otimes m^{*}\right) \circ s \circ m^{*},
$$

where $m$ denotes the multiplication $\times: R \otimes R \longrightarrow R$ and $s: R \otimes R \longrightarrow R \otimes R$ the extension of the map defined on representations by $s: \tau_{1} \otimes \tau_{2} \longmapsto \tau_{2} \otimes \tau_{1}$. Then

$$
\mu^{*}(\tau \rtimes \pi)=M^{*}(\tau) \rtimes \mu^{*}(\pi),
$$

where $\rtimes$ on the right-hand side is determined by $\left(\tau_{1} \otimes \tau_{2}\right) \rtimes(\tau \otimes \theta)=\left(\tau_{1} \times \tau\right) \otimes\left(\tau_{2} \rtimes \theta\right)$.

We now take a moment to review cuspidal reducibility values. Suppose $\rho$ is an irreducible unitary supercuspidal representation of a general linear group and $\sigma$ an irreducible supercuspidal representation of a classical group. If $\rho \neq \check{\rho}$, then $\nu^{x} \rho \rtimes \sigma$ is irreducible for all $x \in \mathbb{R}$; if $\rho \cong \check{\rho}$, then there is a unique nonnegative $x \in \mathbb{R}$ such that $\nu^{x} \rho \rtimes \sigma$ reduces ( $\mathrm{Si2}$; also Corollary 4.4 of [B-J1] for the orthogonal case), which we denote by $\operatorname{red}(\rho ; \sigma)$. The values for $\operatorname{red}(\rho ; \sigma)$ for $S p(2 n, F)$ and $S O(2 n+1, F)$ have been determined (assuming certain conjectures) in [Mœ1] and [Zh]; in the generic case, it is known that they must lie in $\left\{0, \frac{1}{2}, 1\right\}$ ([Sh1], [Sh2]). Further, in the quasi-split, characteristic zero case, the reducibility values are now known to be half-integral ([Art], Mœ4]).

We next review the Casselman criterion for $S(n, F)$ (see [Ca, Wa, which extends easily to the nonconnected group $O(2 n, F))$. Suppose $\pi$ is an irreducible representation of $S(n, F)$. Suppose $\nu^{x_{1}} \rho_{1} \otimes \cdots \otimes \nu^{x_{k}} \rho_{k} \otimes \sigma \leq r_{M, G}(\pi)$ has $\rho_{i}$ an irreducible unitary supercuspidal representation of $G L\left(m_{i}, F\right)$ for $i=1, \ldots, k, \sigma$ an irreducible supercuspidal representation of $S(m, F)$, and $x_{1}, \ldots, x_{k} \in \mathbb{R}$. The Casselman criterion tells us that if $\pi$ is tempered, the following hold:

$$
\begin{aligned}
m_{1} x_{1} & \geq 0 \\
m_{1} x_{1}+m_{2} x_{2} & \geq 0 \\
\vdots & \\
m_{1} x_{1}+m_{2} x_{2}+\cdots+m_{k} x_{k} & \geq 0 .
\end{aligned}
$$

Conversely, if these inequalities hold for any such $\nu^{x_{1}} \rho_{1} \otimes \cdots \otimes \nu^{x_{k}} \rho_{k} \otimes \sigma$ (i.e., $\rho_{i}$ an irreducible unitary supercuspidal representation of $G L\left(m_{i}, F\right)$ and $\sigma$ an irreducible supercuspidal representation of $S(m, F)$ ) appearing in a Jacquet module of $\pi$, then $\pi$ is tempered. The criterion for square-integrability is the same except that the inequalities are strict.

We also take a moment to review the Langlands classification ([B-W], Si1], [Kon; also the appendix of [B-J1] for the nonconnected group $O(2 n, F))$. We work in the subrepresentation setting of the Langlands classification as it is the most convenient for applying Jacquet module methods. Suppose $\tau_{1}, \ldots, \tau_{k}$ are irreducible 
tempered representations of general linear groups and $x_{1}<\cdots<x_{k}$. Then the induced representation $\nu^{x_{1}} \tau_{1} \times \cdots \times \nu^{x_{k}} \tau_{k}$ has a unique irreducible subrepresentation which we denote $\mathcal{L}\left(\nu^{x_{1}} \tau_{1}, \ldots, \nu^{x_{k}} \tau_{k}\right)$. Every irreducible admissible representation of a general linear group may be written in this way, and the data $\nu^{x_{1}} \tau_{1} \otimes \cdots \otimes \nu^{x_{k}} \tau_{k}$ are unique. Turning to classical groups, if $\tau_{1}, \ldots, \tau_{k}$ are irreducible tempered representations of general linear groups, $\tau$ an irreducible tempered representation of $S(n, F)$, and $x_{1}<\cdots<x_{k}<0$, the representation $\nu^{x_{1}} \tau_{1} \times \cdots \times \nu^{x_{k}} \tau_{k} \rtimes \tau$ has a unique irreducible subrepresentation which we denote $L\left(\nu^{x_{1}} \tau_{1}, \ldots, \nu^{x_{k}} \tau_{k} ; \tau\right)$. Further, any irreducible admissible representation of a classical group may be written in this way, and the data $\nu^{x_{1}} \tau_{1} \otimes \cdots \otimes \nu^{x_{k}} \tau_{k} \otimes \tau$ are again unique.

The next lemma is Lemma 5.5 of [J2].

Lemma 2.2. Suppose $\pi$ is an irreducible representation of $G, \lambda$ an irreducible representation of $M$, and $\pi \hookrightarrow i_{G, M}(\lambda)$. If $L>M$, then there is an irreducible representation $\rho$ of $L$ such that

(1) $\pi \hookrightarrow i_{G, L}(\rho)$.

(2) $\rho$ is a subquotient of $i_{L, M}(\lambda)$.

Recall that two segments $\Sigma_{1}, \Sigma_{2}$ are called linked if $\Sigma_{1} \not \subset \Sigma_{2}, \Sigma_{2} \not \subset \Sigma_{1}$, and $\Sigma_{1} \cup \Sigma_{2}$ is also a segment (section 4.1 of [Ze]).

Lemma 2.3. Consider $\delta\left(\Sigma_{1}\right) \times \cdots \times \delta\left(\Sigma_{k}\right)$ with $\Sigma_{1}, \ldots, \Sigma_{k}$ segments. Then

$$
\mathcal{L}\left(\Sigma_{1}^{\prime}, \ldots, \Sigma_{k^{\prime}}^{\prime}\right) \leq \delta\left(\Sigma_{1}\right) \times \cdots \times \delta\left(\Sigma_{k}\right)
$$

if and only if $\Sigma_{1}^{\prime}, \ldots, \Sigma_{k^{\prime}}^{\prime}$ may be obtained from $\Sigma_{1}, \ldots, \Sigma_{k}$ by a sequence of operations which replace linked segments $\Sigma^{*}, \Sigma^{* *}$ with $\left(\Sigma^{*} \cup \Sigma^{* *}\right),\left(\Sigma^{*} \cap \Sigma^{* *}\right)$ (noting that as $\Sigma^{*} \cap \Sigma^{* *}$ may be empty, we may have $\left.k^{\prime}<k\right)$.

Proof. This follows from Theorem 7.1 of [Ze] (see Proposition 3.2.4 of [J5]).

The following is Lemma 2.3 of [J8].

Lemma 2.4. Suppose $f_{\pi}\left(\nu^{x} \rho\right)=f$. If

$$
\pi \hookrightarrow\left(\nu^{x} \rho\right)^{f} \times \lambda_{1} \times \cdots \times \lambda_{k} \rtimes T
$$

with $\lambda_{1} \otimes \cdots \otimes \lambda_{k} \otimes T$ satisfying the conditions for Langlands data (subrepresentation setting), then

$$
\mu_{\left\{\nu^{x} \rho\right\}}^{*}(\pi)=\left(\nu^{x} \rho\right)^{f} \otimes L\left(\lambda_{1}, \ldots, \lambda_{k} ; T\right)
$$

(up to multiplicity if $x=0$ ).

2.2. The extended Mœglin-Tadić classification. In this section, we review the extension of the construction of [M-T] to tempered representations. The extension used here is from [J7]; we also note the somewhat different extension available in T5].

Recall that the Mœglin-Tadić classification is a bijective correspondence between (equivalence classes of) discrete series for a family of classical groups and (equivalence classes of) admissible triples. An admissible triple is a triple of the form $(J o r d, \sigma, \varepsilon)$. Here Jord consists of pairs $(\rho, a)$, with $\rho$ an irreducible unitary supercuspidal representation of a general linear group and $a \in \mathbb{N}$ subject to a parity condition from $\rho, \sigma$ the "partial cuspidal support" (the supercuspidal representation of a classical group which appears in any minimal nonzero Jacquet module term), and $\varepsilon$ a function defined on a subset of $\operatorname{Jord} \cup(\operatorname{Jord} \times \operatorname{Jord})$ taking values in $\{ \pm 1\}$ 
which essentially distinguishes between discrete series having the same supercuspidal support. Information about induced representations into which the discrete series embeds is also encoded in the data. This classification and its properties have been summarized in [M-T], many of the references for this paper (e.g., [T3], [Mu2], [Mu3, T4, JJ6, T5], [J7]), as well as many other places. We forgo doing so again and simply refer the reader to these sources, as well as to the characterization of admissibility (in the [M-T] sense) in the appendix.

To extend the Mœglin-Tadić classification to tempered representations, we first consider the elliptic case. Suppose

$$
T_{\text {ell }} \hookrightarrow \delta\left(\left[\nu^{\frac{-c_{1}+1}{2}} \rho_{1}, \nu^{\frac{c_{1}-1}{2}} \rho_{1}\right]\right) \times \cdots \times \delta\left(\left[\nu^{\frac{-c_{\ell}+1}{2}} \rho_{\ell}, \nu^{\frac{c_{\ell}-1}{2}} \rho_{\ell}\right]\right) \rtimes \delta,
$$

with $\delta$ a discrete series for a classical group. Let $\left(\operatorname{Jord}(\delta), \sigma, \varepsilon_{\delta}\right)$ be the MøglinTadić data for $\delta$, with $S_{\delta} \subset \operatorname{Jord}(\delta) \cup(\operatorname{Jord}(\delta) \times \operatorname{Jord}(\delta))$ the domain for $\varepsilon_{\delta}$. Intuitively, we construct $\operatorname{Jord}(T)$ from $\operatorname{Jord}(\delta)$ by adding two copies each of $\left(\rho_{1}, c_{1}\right), \ldots$, $\left(\rho_{\ell}, c_{\ell}\right)$ (one for each end of $\delta\left(\left[\nu^{\frac{-c_{i}+1}{2}} \rho_{i}, \nu^{\frac{c_{i}-1}{2}} \rho_{i}\right]\right)$, even if $\left.c_{i}=1\right)$. Thus we introduce a fourth datum, $m_{T}$ - the multiplicity — so have $T$ associated to $\left(\operatorname{Jord}(T), \sigma, \varepsilon_{T}, m_{T}\right)$. Thus,

$$
\operatorname{Jord}(T)=\operatorname{Jord}(\delta) \cup\left\{\left(\rho_{1}, c_{1}\right), \ldots,\left(\rho_{\ell}, c_{\ell}\right)\right\}
$$

and

$$
m_{T}(\rho, a)=\left\{\begin{array}{l}
1 \text { if }(\rho, a) \in \operatorname{Jord}(\delta), \\
2 \text { if }(\rho, a) \cong\left(\rho_{i}, c_{i}\right) \text { for some } i .
\end{array}\right.
$$

Again, we have $\varepsilon_{T}: S_{T} \longrightarrow\{ \pm 1\}$, with the domain

$$
S_{T} \subset \operatorname{Jord}(T) \cup(\operatorname{Jord}(T) \times \operatorname{Jord}(T)) .
$$

We have $S_{T} \supset S_{\delta}$, and $\left.\varepsilon_{T}\right|_{S_{\delta}}=\varepsilon_{\delta}$. The additional values of $\varepsilon_{T}$ effectively distinguish the $2^{\ell}$ components of $\delta\left(\left[\nu^{\frac{-c_{1}+1}{2}} \rho_{1}, \nu^{\frac{c_{1}-1}{2}} \rho_{1}\right]\right) \times \cdots \times \delta\left(\left[\nu^{\frac{-c_{\ell}+1}{2}} \rho_{\ell}, \nu^{\frac{c_{\ell}-1}{2}} \rho_{\ell}\right]\right) \rtimes \delta$. In particular, we have the following extension of the basic embedding property of $\left[\mathrm{M}-\mathrm{T}\right.$ : for $(\rho, a) \in \operatorname{Jord}(T)$, let $a_{-}$be the largest value of $b<a$ satisfying $(\rho, b) \in \operatorname{Jord}(\delta)$ if it exists. Then (Proposition 2.3.2 of [J7])

(1) if $m(\rho, a)=1$,

(2) if $m(\rho, a)=2$,

$$
\varepsilon\left((\rho, a),\left(\rho, a_{-}\right)\right)=1 \Leftrightarrow T \hookrightarrow \delta\left(\left[\nu^{\frac{a_{-}+1}{2}} \rho, \nu^{\frac{a-1}{2}} \rho\right]\right) \rtimes \theta \text { for some irreducible } \theta,
$$

$$
\begin{aligned}
\varepsilon\left((\rho, a),\left(\rho, a_{-}\right)\right)=1 & \Leftrightarrow T \hookrightarrow \delta\left(\left[\nu^{\frac{a_{-}+1}{2}} \rho, \nu^{\frac{a-1}{2}} \rho\right]\right) \\
& \times \delta\left(\left[\nu^{\frac{a_{-}+1}{2}} \rho, \nu^{\frac{a-1}{2}} \rho\right]\right) \rtimes \theta \text { for some irreducible } \theta .
\end{aligned}
$$

Other basic properties of the Møglin-Tadić classification also have counterparts in the extension to the tempered case; we forgo including them here but include citations when used.

For more general tempered representations - i.e., no longer assuming elliptic - we construct $\operatorname{Jord}(T), m_{T}$ in a similar manner. Write

$$
T \cong \delta\left(\left[\nu^{\frac{-d_{1}+1}{2}} \rho_{1}^{\prime}, \nu^{\frac{d_{1}-1}{2}} \rho_{1}^{\prime}\right]\right) \times \cdots \times \delta\left(\left[\nu^{\frac{-d_{m}+1}{2}} \rho_{m}^{\prime}, \nu^{\frac{d_{m}-1}{2}} \rho_{m}^{\prime}\right]\right) \rtimes T_{\text {ell }}
$$

(irreducibly induced) with $T_{\text {ell }}$ elliptic tempered. We construct $\operatorname{Jor} d(T), m_{T}$ from $\operatorname{Jord}\left(T_{\ell}\right), m_{T_{e l l}}$ by adding one copy each of $\left(\rho_{1}^{\prime}, d_{1}\right),\left(\check{\rho}^{\prime}{ }_{1}, d_{1}\right), \ldots,\left(\rho_{m}^{\prime}, d_{m}\right),\left(\check{\rho}_{m}^{\prime}, d_{m}\right)$ (so two copies of $\left(\rho_{i}^{\prime}, d_{i}\right)$ are added if $\left.\check{\rho}^{\prime}{ }_{i} \cong \rho_{i}\right) ; S_{T}$ and $\varepsilon_{T}$ match $S_{T_{\text {ell }}}$ and $\varepsilon_{T_{\text {ell }}}$ (noting that the corresponding induced representation is irreducible so we do not have 
components to distinguish). We then have an extension of the above embedding: for $(\rho, a) \in \operatorname{Jord}\left(T_{\text {ell }}\right)$, let $a_{-}<a$ be the largest value such that $\left(\rho, a_{-}\right) \in \operatorname{Jord}\left(T_{\text {ell }}\right)$ if it exists. Then

$$
\varepsilon\left((\rho, a),\left(\rho, a_{-}\right)\right)=1 \Leftrightarrow T \hookrightarrow \underbrace{\delta\left(\left[\nu^{\frac{a_{-}+1}{2}} \rho, \nu^{\frac{a-1}{2}} \rho\right]\right) \times \cdots \times \delta\left(\left[\nu^{\frac{a_{-}+1}{2}} \rho, \nu^{\frac{a-1}{2}} \rho\right]\right)}_{m(\rho, a)} \rtimes \theta .
$$

This is not explicitly stated in [J7], but is a straightforward consequence of (2.2) and the irreducibility of (2.3).

\section{Algorithm FOR $\mu_{\left\{\nu^{x} \rho\right\}}^{*}$}

Let $\pi$ be a smooth irreducible representation of $S(n, F)$. In this section, we give an algorithm for calculating $\mu_{\left\{\nu^{x} \rho\right\}}^{*}(\pi)$. Because of certain technical problems which arise in the case $x=0$ - discussed in detail in section 7 - most of this section applies only when $x \neq 0$. The exception is the results in the tempered case-recalled from J8] in the first subsection-which do allow the possibility $x=0$. The second subsection covers the case $x<0$, while the third addresses the more difficult case $x>0$. The algorithm for $x>0$ reduces to the special case where the representation has the form $\pi \cong L\left(\left(\nu^{\frac{-a+1}{2}} \rho\right)^{k}, \delta\left(\left[\nu^{\frac{-a+1}{2}} \rho, \nu^{\frac{a-3}{2}} \rho\right]\right)^{\ell} ; T\right)$, which is more delicate as it is where the reducibility of $\nu^{\frac{-a+1}{2}} \rho \rtimes T$ enters the picture. That case is covered in the last subsection. We remark that when applying the algorithms of the last two subsections to specific representations, one often has steps which do not apply to those representations, effectively shortening the algorithms.

3.1. The tempered case. In the case where $\pi=T$ is tempered, $\mu_{\left\{\nu^{x} \rho\right\}}^{*}(T)$ may be calculated using the results of [J8], summarized in the following theorem.

Theorem 3.1. Suppose $T$ is irreducible tempered with $(\rho, a) \in \operatorname{Jord}(T)$. If $a>2$, we have the following:

(1) If $\rho \neq \check{\rho}$, then

$\mu_{\left\{\nu \frac{a-1}{2} \rho\right\}}^{*}(T)=\left(\nu^{\frac{a-1}{2}} \rho\right)^{m_{T}(\rho, a)} \otimes L\left(\delta\left(\left[\nu^{\frac{-a+1}{2}} \rho, \nu^{\frac{a-3}{2}} \rho\right]\right)^{m_{T}(\rho, a)} ; T_{1}\right)$,

where $m_{T_{1}}(\rho, a)=m_{T_{1}}(\check{\rho}, a)=0, m_{T_{1}}\left(\rho^{\prime}, b\right)=m_{T}\left(\rho^{\prime}, b\right)$ for all other $\left(\rho^{\prime}, b\right)$, and $\varepsilon_{T_{1}}=\varepsilon_{T}$ (noting that $\left.(\rho, a) \notin S_{T}\right)$.

(2) If $\rho \cong \check{\rho}$ but $\frac{a-1}{2} \not \equiv \operatorname{red}(\rho ; \sigma) \bmod 1$,

$$
\mu_{\left\{\nu \frac{a-1}{2} \rho\right\}}^{*}(T)=\left(\nu^{\frac{a-1}{2}} \rho\right)^{m_{T}(\rho, a)} \otimes T_{2},
$$

where $m_{T_{2}}(\rho, a)=0, m_{T_{2}}(\rho, a-2)=m_{T}(\rho, a), m_{T_{2}}\left(\rho^{\prime}, b\right)=m_{T}\left(\rho^{\prime}, b\right)$ for all other $\left(\rho^{\prime}, b\right)$, and $\varepsilon_{T_{2}}=\varepsilon_{T}$ (noting that $\left.(\rho, a) \notin S_{T}\right)$.

(3) If $\rho \cong \check{\rho}$ with $\frac{a-1}{2} \equiv \operatorname{red}(\rho ; \sigma) \bmod 1$ and either (i) $a>a_{-}+2$, or (ii) $\varepsilon_{T}(\rho, a) \varepsilon_{T}\left(\rho, a_{-}\right)^{-1}=1$, then

$$
\mu_{\left\{\nu \frac{a-1}{2} \rho\right\}}^{*}(T)=\left(\nu^{\frac{a-1}{2}} \rho\right)^{m_{T}(\rho, a)} \otimes T_{3},
$$

where $m_{T_{3}}(\rho, a)=0, m_{T_{3}}(\rho, a-2)=m_{T}(\rho, a)+m_{T}(\rho, a-2)$, and $m_{T_{2}}\left(\rho^{\prime}, b\right)$ $=m_{T}\left(\rho^{\prime}, b\right)$ for all other $\left(\rho^{\prime}, b\right)$. If $m_{T}(\rho, a-2)>0, \varepsilon_{T_{3}}$ is just given by restriction; if $m_{T}(\rho, a-2)=0$, it is given by substituting $(\rho, a-2)$ for $(\rho, a)$. More precisely, if $m(\rho, a-2)=0, \varepsilon_{T_{3}}$ is determined by the following 
changes: $\varepsilon_{T_{3}}(\rho, a-2)=\varepsilon_{T}(\rho, a)$ if defined, and $\varepsilon_{T_{3}}(\rho, a-2) \varepsilon_{T_{3}}(\rho, b)^{-1}=$ $\varepsilon_{T}(\rho, a) \varepsilon_{T}(\rho, b)^{-1}$.

(4) If $\rho \cong \check{\rho}$ with $\frac{a-1}{2} \equiv \operatorname{red}(\rho ; \sigma) \bmod 1, a_{-}=a-2$, and $\varepsilon_{T}(\rho, a) \varepsilon_{T}\left(\rho, a_{-}\right)^{-1}=$ -1 , then

$$
\mu_{\left\{\nu^{\frac{a-1}{2}} \rho\right\}}^{*}(T)=\left\{\begin{array}{l}
\left(\nu^{\frac{a-1}{2}} \rho\right)^{m_{T}(\rho, a)-1} \otimes T_{4} \text { if } m_{T}(\rho, a) \text { odd }, \\
\left(\nu^{\frac{a-1}{2}} \rho\right)^{m_{T}(\rho, a)-1} \otimes L\left(\delta\left(\left[\nu^{\frac{-a+1}{2}} \rho, \nu^{\frac{a-3}{2}} \rho\right]\right) ; T_{5}\right) \text { if } m_{T}(\rho, a) \text { even } .
\end{array}\right.
$$

Here, $m_{T_{4}}(\rho, a)=1, m_{T_{4}}(\rho, a-2)=m_{T}(\rho, a-2)+m_{T}(\rho, a)-1, m_{T_{4}}\left(\rho^{\prime}, b\right)=$ $m_{T}\left(\rho^{\prime}, b\right)$ for all other $\left(\rho^{\prime}, b\right)$, and $\varepsilon_{T_{4}}=\varepsilon_{T} ;$ for $T_{5}, m_{T_{5}}(\rho, a)=0$, $m_{T_{5}}(\rho, a-2)=m_{T}(\rho, a-2)+m_{T}(\rho, a)-2, m_{T_{5}}\left(\rho^{\prime}, b\right)=m_{T}\left(\rho^{\prime}, b\right)$ for all other $\left(\rho^{\prime}, b\right)$, and $\varepsilon_{T_{5}}$ the restriction of $\varepsilon_{T}$.

If $a=2$, we have the following:

(5) If $\rho \neq \check{\rho}$, then

$$
\mu_{\left\{\nu^{\frac{1}{2}} \rho\right\}}^{*}(T)=\left(\nu^{\frac{1}{2}} \rho\right)^{m_{T}(\rho, 2)} \otimes L\left(\left(\nu^{-\frac{1}{2}} \rho\right)^{m_{T}(\rho, 2)} ; T_{1}\right),
$$

where $m_{T_{1}}(\rho, a)=m_{T_{1}}(\check{\rho}, a)=0, m_{T_{1}}\left(\rho^{\prime}, b\right)=m_{T}\left(\rho^{\prime}, b\right)$ for all other $\left(\rho^{\prime}, b\right)$, and $\varepsilon_{T_{1}}=\varepsilon_{T}$ (noting that $\left.(\rho, 2) \notin S_{T}\right)$.

(6) If $\rho \cong \check{\rho}$ but $\operatorname{red}(\rho ; \sigma) \not \equiv \frac{1}{2} \bmod 1$,

$$
\mu_{\left\{\nu^{\frac{1}{2}} \rho\right\}}^{*}(T)=\left(\nu^{\frac{1}{2}} \rho\right)^{m_{T}(\rho, 2)} \otimes T_{2},
$$

where $m_{T_{2}}(\rho, 2)=0, m_{T_{2}}\left(\rho^{\prime}, b\right)=m_{T}\left(\rho^{\prime}, b\right)$ for all other $\left(\rho^{\prime}, b\right)$, and $\varepsilon_{T_{2}}=$ $\varepsilon_{T}$ (noting that $\left.(\rho, a) \notin S_{T}\right)$.

(7) If $\rho \cong \check{\rho}$ with $\operatorname{red}(\rho ; \sigma) \equiv \frac{1}{2} \bmod 1$ and $\varepsilon_{T}(\rho, 2)=1$, then

$$
\mu_{\left\{\nu^{\frac{1}{2}} \rho\right\}}^{*}(T)=\left(\nu^{\frac{1}{2}} \rho\right)^{m_{T}(\rho, 2)} \otimes T_{3},
$$

where $m_{T_{3}}(\rho, 2)=0, m_{T_{2}}\left(\rho^{\prime}, b\right)=m_{T}\left(\rho^{\prime}, b\right)$ for all other $\left(\rho^{\prime}, b\right)$, and $\varepsilon_{T 3}$ just the restriction of $\varepsilon_{T}$.

(8) If $\rho \cong \check{\rho}$ with $\operatorname{red}(\rho ; \sigma) \equiv \frac{1}{2} \bmod 1$ and $\varepsilon_{T}(\rho, 2)=-1$, then

$$
\mu_{\left\{\nu^{\frac{1}{2}} \rho\right\}}^{*}(T)=\left\{\begin{array}{l}
\left(\nu^{\frac{1}{2}} \rho\right)^{m_{T}(\rho, 2)-1} \otimes T_{4} \text { if } m_{T}(\rho, 2) \text { odd } \\
\left(\nu^{\frac{1}{2}} \rho\right)^{m_{T}(\rho, 2)-1} \otimes L\left(\nu^{-\frac{1}{2}} \rho ; T_{5}\right) \text { if } m_{T}(\rho, 2) \text { even. }
\end{array}\right.
$$

Here, $m_{T_{4}}(\rho, 2)=1, m_{T_{4}}\left(\rho^{\prime}, b\right)=m_{T}\left(\rho^{\prime}, b\right)$ for all other $\left(\rho^{\prime}, b\right)$, and $\varepsilon_{T_{4}}=$ $\varepsilon_{T} ;$ for $T_{5}, m_{T_{5}}(\rho, 2)=0, m_{T_{5}}\left(\rho^{\prime}, b\right)=m_{T}\left(\rho^{\prime}, b\right)$ for all other $\left(\rho^{\prime}, b\right)$, and $\varepsilon_{T_{5}}$ just the restriction of $\varepsilon_{T}$.

If $a=1$, we have the following:

(9) If $\rho \neq \check{\rho}$, then

$$
\mu_{\{\rho\}}^{*}(T)=(\rho)^{m_{T}(\rho, 1)} \otimes T_{1},
$$

where $m_{T_{1}}(\rho, 1)=m_{T_{1}}(\check{\rho}, 1)=0, m_{T_{1}}\left(\rho^{\prime}, b\right)=m_{T}\left(\rho^{\prime}, b\right)$ for all other $\left(\rho^{\prime}, b\right)$, and $\varepsilon_{T_{1}}=\varepsilon_{T}$ (noting that $\left.(\rho, 1) \notin S_{T}\right)$.

(10) If $\rho \cong \check{\rho}$ but $\operatorname{red}(\rho ; \sigma) \not \equiv 0 \bmod 1$,

$$
\mu_{\{\rho\}}^{*}(T)=2^{\frac{m_{T}(\rho, 1)}{2}} \cdot(\rho)^{\frac{m_{T}(\rho, 1)}{2}} \otimes T_{2},
$$

where $m_{T_{2}}(\rho, 1)=0, m_{T_{2}}\left(\rho^{\prime}, b\right)=m_{T}\left(\rho^{\prime}, b\right)$ for all other $\left(\rho^{\prime}, b\right)$, and $\varepsilon_{T_{2}}=$ $\varepsilon_{T}$ (noting that $\left.(\rho, 1) \notin S_{T}\right)$. 
(11) If $\rho \cong \check{\rho}$ with $\operatorname{red}(\rho ; \sigma) \equiv 0 \bmod 1$, then

$$
\mu_{\{\rho\}}^{*}(T)=\left\{\begin{array}{l}
2^{\frac{m_{T}(\rho, 1)}{2}} \cdot(\rho)^{\frac{m_{T}(\rho, 1)}{2}} \otimes T_{3} \text { if } m_{T}(\rho, 1) \text { even, } \\
2^{\frac{m_{T}(\rho, 1)-1}{2}} \cdot(\rho)^{\frac{m_{T}(\rho, 1)-1}{2}} \otimes T_{3} \text { if } m_{T}(\rho, 1) \text { odd },
\end{array}\right.
$$

where

$$
m_{T_{3}}(\rho, 1)= \begin{cases}0 & \text { if } m_{T}(\rho, 1) \text { even }, \\ 1 & \text { if } m_{T}(\rho, 1) \text { odd },\end{cases}
$$

$m_{T_{3}}\left(\rho^{\prime}, b\right)=m_{T}\left(\rho^{\prime}, b\right)$ for all other $\left(\rho^{\prime}, b\right)$, and $\varepsilon_{T_{3}}=\varepsilon_{T}$ if $m_{T}(\rho, 1)$ odd, $\varepsilon_{T_{3}}$ the restriction of $\varepsilon_{T}$ if $m_{T}(\rho, 1)$ even.

3.2. Calculating $\mu_{\left\{\nu^{x} \rho\right\}}^{*}(\pi)$ in the case $x<0$. Now, write $\pi=L(\Delta ; T)$. Then $\pi \hookrightarrow \mathcal{L}(\Delta) \rtimes T$. Considering $\mu_{\left\{\nu^{x} \rho\right\}}^{*}(\mathcal{L}(\Delta) \rtimes T)$, we see three possible sources for copies of $\nu^{x} \rho$ :

(1) terms $\nu^{x} \rho \otimes \ldots$ in $r_{\min }((\mathcal{L}(\Delta))$

(2) terms $\cdots \otimes \nu^{-x} \tilde{\rho}$ in $r_{\min }(\mathcal{L}(\Delta))$,

(3) terms $\nu^{x} \rho \otimes \ldots$ in $r_{\min }(T)$.

Notice, however, that since segments appearing in $\Delta$ all have negative central exponent, (2) cannot contribute unless $x>0$. Similarly, by the Casselman criterion, (3) cannot contribute unless $x \geq 0$. We address the simplest case - namely, $x<0$ here, deferring the rest until later sections.

We now turn to the task of calculating $\mu_{\left\{\nu^{x} \rho\right\}}^{*}(\pi)$ when $x<0$. Write

$$
\pi=L(\Delta ; T) \hookrightarrow \mathcal{L}(\Delta) \rtimes T .
$$

If $m_{\left\{\nu^{x} \rho\right\}}^{*}(\mathcal{L}(\Delta))=\left(\nu^{x} \rho\right)^{f} \otimes \mathcal{L}\left(\Delta^{\prime}\right)$ - which may be calculated by Theorem 2.2.1 of [J5]-then

$$
\begin{gathered}
\mathcal{L}(\Delta) \hookrightarrow\left(\nu^{x} \rho\right)^{f} \times \mathcal{L}\left(\Delta^{\prime}\right) \\
\Downarrow \\
\pi \hookrightarrow\left(\nu^{x} \rho\right)^{f} \times \mathcal{L}\left(\Delta^{\prime}\right) \rtimes T .
\end{gathered}
$$

We claim that (i) $f_{\pi}\left(\nu^{x} \rho\right)=f$, and (ii) $\Delta^{\prime} \otimes T$ satisfies the conditions for Langlands data. Assuming these for the moment, it then follows from Lemma 2.4 that

$$
\mu_{\left\{\nu^{x} \rho\right\}}^{*}(\pi)=\left(\nu^{x} \rho\right)^{f} \otimes L\left(\Delta^{\prime} ; T\right) .
$$

To check that (i) and (ii) hold, note that $\Delta^{\prime}$ is obtained from $\Delta$ by removing copies of $\nu^{x} \rho$ from the upper ends of segments in $\Delta$ (see Theorem 2.2.1 of [J5] for a more precise description, noting the convention on rearrangement into Langlands data order in that paper). Since such a removal lowers the central exponent of a segment, we see that the segments in $\Delta^{\prime}$ all have negative central exponents. In particular, this tells us (ii) holds.

For (i), it follows immediately from Frobenius reciprocity that $f_{\pi}\left(\nu^{x} \rho\right) \geq f$. On the other hand, observe that $f_{T}\left(\nu^{x} \rho\right)=0$ by the Casselman criterion. By construction, $f_{\mathcal{L}\left(\Delta^{\prime}\right)}\left(\nu^{x} \rho\right)=0$; as $\delta^{\prime}$ has segments with negative central exponents (hence lower segment ends must have negative exponents), $g_{\mathcal{L}\left(\Delta^{\prime}\right)}\left(\nu^{-x} \check{\rho}\right)=0$. Therefore, $f_{\mathcal{L}\left(\Delta^{\prime}\right) \rtimes T}\left(\nu^{x} \rho\right)=0$. It then follows that

$$
f_{\pi}\left(\nu^{x} \rho\right) \leq f+f_{\mathcal{L}\left(\Delta^{\prime}\right) \rtimes T}\left(\nu^{x} \rho\right)=f,
$$

implying $f_{\pi}\left(\nu^{x} \rho\right)=f$, as claimed. 
Note 3.2. In the reverse direction, if one has

$$
\mu_{\left\{\nu^{x} \rho\right\}}^{*}(\pi)=\left(\nu^{x} \rho\right)^{f} \otimes \theta
$$

for $x<0$ and the goal is to identify $\pi$, write $\theta=L\left(\Delta^{\prime} ; T\right)$. From [J5, we may determine $\mathcal{L}(\Delta)$ such that $m_{\left\{\nu^{x} \rho\right\}}^{*}(\mathcal{L}(\Delta))=\left(\nu^{x} \rho\right)^{f} \otimes \mathcal{L}\left(\Delta^{\prime}\right)$. We then have $\pi=$ $L(\Delta ; T)$.

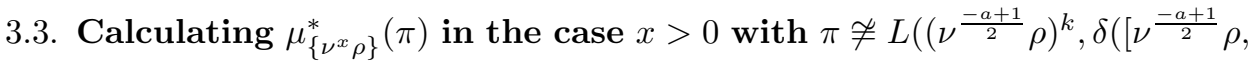
$\left.\left.\left.\nu^{\frac{a-3}{2}} \rho\right]\right)^{\ell} ; T\right)$. First, write $\mathcal{L}(\Delta)=\mathcal{L}\left(\nu^{x_{1}} \tau_{1}, \ldots, \nu^{x_{k-1}} \tau_{k-1}, \nu^{-\frac{1}{2}} \tau_{k}\right)$ and

$$
\nu^{-\frac{1}{2}} \tau_{k}=\nu^{-\frac{1}{2}} \tau_{k}^{\prime} \times \delta\left(\left[\nu^{\frac{-a+1}{2}} \rho, \nu^{\frac{a-3}{2}} \rho\right]\right)^{\ell}
$$

(with $\ell$ maximal). If $\Delta^{(1)}=\nu^{x_{1}} \tau_{1} \otimes \cdots \otimes \nu^{x_{k-1}} \tau_{k-1} \otimes \nu^{-\frac{1}{2}} \tau_{k}^{\prime}$, it follows from the Langlands classification that $\mathcal{L}(\Delta) \hookrightarrow \mathcal{L}\left(\Delta^{(1)}\right) \times \delta\left(\left[\nu^{\frac{-a+1}{2}} \rho, \nu^{\frac{a-3}{2}} \rho\right]\right)^{\ell}$. Then,

$$
\pi \hookrightarrow \mathcal{L}\left(\Delta^{(1)}\right) \times \delta\left(\left[\nu^{\frac{-a+1}{2}} \rho, \nu^{\frac{a-3}{2}} \rho\right]\right)^{\ell} \rtimes T .
$$

Further, writing ${ }_{\left\{\nu \frac{-a+1}{2} \rho\right\}} m^{*}\left(\mathcal{L}\left(\Delta^{(1)}\right)=\mathcal{L}\left(\Delta^{(2)}\right) \otimes\left(\nu^{\frac{-a+1}{2}} \rho\right)^{k}\right.$ (see Theorem 2.4.5 of [J5]), we have

$$
\begin{gathered}
\pi \hookrightarrow \mathcal{L}\left(\Delta^{(2)}\right) \times\left(\nu^{\frac{-a+1}{2}} \rho\right)^{k} \times \delta\left(\left[\nu^{\frac{-a+1}{2}} \rho, \nu^{\frac{a-3}{2}} \rho\right]\right)^{\ell} \rtimes T \\
\Downarrow(\operatorname{Lemma} 2.2) \\
\pi \hookrightarrow \mathcal{L}\left(\Delta^{(2)}\right) \rtimes \lambda
\end{gathered}
$$

for some irreducible $\lambda \leq\left(\nu^{\frac{-a+1}{2}} \rho\right)^{k} \times \delta\left(\left[\nu^{\frac{-a+1}{2}} \rho, \nu^{\frac{a-3}{2}} \rho\right]\right)^{\ell} \rtimes T$.

Claim 1. $\lambda=L\left(\left(\nu^{\frac{-a+1}{2}} \rho\right)^{k}, \delta\left(\left[\nu^{\frac{-a+1}{2}} \rho, \nu^{\frac{a-3}{2}} \rho\right]\right)^{\ell} ; T\right)$.

We verify this in Lemma 5.4 below. Continuing, write

$$
\mu_{\left\{\nu \frac{a-1}{2} \rho\right\}}^{*}\left(L\left(\left(\nu^{\frac{-a+1}{2}} \rho\right)^{k}, \delta\left(\left[\nu^{\frac{-a+1}{2}} \rho, \nu^{\frac{a-3}{2}} \rho\right]\right)^{\ell} ; T\right)\right)=\left(\nu^{\frac{a-1}{2}} \rho\right)^{f_{1}} \otimes \theta_{1},
$$

where the (nontrivial) calculation required for this is discussed in section 3.4. Then,

$$
\begin{aligned}
\pi \hookrightarrow \mathcal{L}\left(\Delta^{(2)}\right) & \times\left(\nu^{\frac{a-1}{2}} \rho\right)^{f_{1}} \rtimes \theta_{1} \\
& \Downarrow(\text { Lemma 2.2) } \\
\pi & \hookrightarrow \lambda^{\prime} \rtimes \theta_{1}
\end{aligned}
$$

for some irreducible $\lambda^{\prime} \leq \mathcal{L}\left(\Delta^{(2)}\right) \times\left(\nu^{\frac{a-1}{2}} \rho\right)^{f_{1}}$.

Claim 2. $\lambda^{\prime}=\mathcal{L}\left(\Delta^{(2)},\left(\nu^{\frac{a-1}{2}} \rho\right)^{f_{1}}\right)$.

This is verified in Lemma 5.5 Note that since $f_{\theta_{1}}\left(\nu^{\frac{a-1}{2}} \rho\right)=0$ and $g_{\mathcal{L}\left(\Delta^{(2)}\right)}\left(\nu^{\frac{-a+1}{2}} \rho\right)$ $=0 \Rightarrow g_{\mathcal{L}\left(\Delta^{(2)},\left(\nu^{\frac{a-1}{2}} \rho\right)^{f_{1}}\right)}\left(\nu^{\frac{-a+1}{2}} \rho\right)=0$ (noting $x=\frac{a-1}{2}>0$ ), we now have

$$
f_{\pi}\left(\nu^{\frac{a-1}{2}} \rho\right)=f_{\mathcal{L}\left(\Delta^{(2)},\left(\nu^{\frac{a-1}{2}} \rho\right)^{f_{1}}\right)}\left(\nu^{\frac{a-1}{2}} \rho\right) .
$$

Write (see Theorem 2.2.1 of [J5])

$$
m_{\left\{\nu \frac{a-1}{2} \rho\right\}}^{*}\left(\mathcal{L}\left(\Delta^{(2)},\left(\nu^{\frac{a-1}{2}} \rho\right)^{f_{1}}\right)\right)=\left(\nu^{\frac{a-1}{2}} \rho\right)^{f} \otimes \mathcal{L}\left(\Delta^{(3)}\right),
$$

with $f=f_{\pi}\left(\nu^{\frac{a-1}{2}} \rho\right)$ from above. We now have

$$
\pi \hookrightarrow\left(\nu^{\frac{a-1}{2}} \rho\right)^{f} \times \mathcal{L}\left(\Delta^{(3)}\right) \rtimes \theta_{1} .
$$


Further, it follows from the algorithm for computing $m_{\left\{\nu^{\frac{a-1}{2}} \rho\right\}}^{*}\left(\mathcal{L}\left(\Delta^{(2)},\left(\nu^{\frac{a-1}{2}} \rho\right)^{f_{1}}\right)\right)$ (essentially, the $\nu^{\frac{a-1}{2}} \rho$ are drawn from the upper segment ends in $\Delta^{(2)} \otimes\left(\nu^{\frac{a-1}{2}} \rho\right)^{f_{1}}$ ) that $\Delta^{(3)}$ has the form $\Delta^{(4)} \otimes\left(\nu^{\frac{a-1}{2}} \rho\right)^{f_{2}}$ for some $f_{2} \leq f_{1}$ and $\Delta^{(4)}$ consisting of segments with negative central exponent. In particular, it then follows that

$$
\begin{gathered}
\pi \hookrightarrow\left(\nu^{\frac{a-1}{2}} \rho\right)^{f} \times \mathcal{L}\left(\Delta^{(4)}\right) \times\left(\nu^{\frac{a-1}{2}} \rho\right)^{f_{2}} \rtimes \theta_{1} \\
\Downarrow(\text { Lemma } 2.2) \\
\pi \hookrightarrow\left(\nu^{\frac{a-1}{2}} \rho\right)^{f} \times \mathcal{L}\left(\Delta^{(4)}\right) \rtimes \theta_{2}
\end{gathered}
$$

for some irreducible $\theta_{2} \leq\left(\nu^{\frac{a-1}{2}} \rho\right)^{f_{2}} \rtimes \theta_{1}$.

Claim 3. $f_{\theta_{2}}\left(\nu^{\frac{a-1}{2}} \rho\right)=f_{2}$.

This is verified in Lemma 5.6. Now, it follows from Proposition 5.1 and Corollary 5.2 that $\theta_{2}$ has the form

$$
\theta_{2}=L\left(\left(\nu^{\frac{-a+1}{2}} \rho\right)^{k_{\theta_{2}}}, \delta\left(\left[\nu^{\frac{-a+1}{2}} \rho, \nu^{\frac{a-3}{2}} \rho\right]\right)^{\ell_{\theta_{2}}} ; T_{\theta_{2}}\right) .
$$

Therefore, we may use the results of section 3.4 to determine $k_{\theta_{2}}, \ell_{\theta_{2}}, T_{\theta_{2}}$ so that $\mu_{\left\{\nu \frac{a-1}{2} \rho\right\}}^{*}\left(\theta_{2}\right)=\left(\nu^{\frac{a-1}{2}} \rho\right)^{f_{2}} \otimes \theta_{1}$. It then follows that

$$
\begin{aligned}
& \pi \hookrightarrow\left(\nu^{\frac{a-1}{2}} \rho\right)^{f} \times \mathcal{L}\left(\Delta^{(4)}\right) \times\left(\nu^{\frac{-a+1}{2}} \rho\right)^{k_{\theta_{2}}} \rtimes L\left(\delta\left(\left[\nu^{\frac{-a+1}{2}} \rho, \nu^{\frac{a-3}{2}} \rho\right]\right)^{\ell_{\theta_{2}}} ; T_{\theta_{2}}\right) \\
& \Downarrow \text { (Lemma 2.2) } \\
& \pi \hookrightarrow\left(\nu^{\frac{a-1}{2}} \rho\right)^{f} \times \mathcal{L}\left(\Delta^{(5)}\right) \rtimes L\left(\delta\left(\left[\nu^{\frac{-a+1}{2}} \rho, \nu^{\frac{a-3}{2}} \rho\right]\right)^{\ell_{\theta_{2}}} ; T_{\theta_{2}}\right)
\end{aligned}
$$

for some irreducible $\mathcal{L}\left(\Delta^{(5)}\right) \leq \mathcal{L}\left(\Delta^{(4)}\right) \times\left(\nu^{\frac{-a+1}{2}} \rho\right)^{k_{\theta_{2}}}$.

Claim 4. $g_{\mathcal{L}\left(\Delta^{(5)}\right)}\left(\nu^{\frac{-a+1}{2}} \rho\right)=k_{\theta_{2}}$.

This is proved in Lemma 5.7 below. Finally, using Theorem 2.4.5 and (the analogue of) Remark 2.2.4 of [J5], we may determine $\Delta^{(5)}$ such that ${ }_{\left\{\nu \frac{-a+1}{2}\right.}{ }_{\rho\}} m^{*}\left(\mathcal{L}\left(\Delta^{(5)}\right)\right.$ $=\mathcal{L}\left(\Delta^{(4)}\right) \otimes\left(\nu^{\frac{-a+1}{2}} \rho\right)^{k_{\theta_{2}}}$. Note that it follows immediately from the procedure for

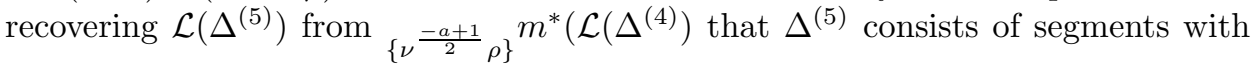
negative central exponents. Thus,

$$
\begin{gathered}
\pi \hookrightarrow\left(\nu^{\frac{a-1}{2}} \rho\right)^{f} \times \mathcal{L}\left(\Delta^{(5)}\right) \rtimes L\left(\delta\left(\left[\nu^{\frac{-a+1}{2}} \rho, \nu^{\frac{a-3}{2}} \rho\right]\right)^{\ell_{\theta_{2}}} ; T_{\theta_{2}}\right) \\
\Downarrow(\operatorname{Lemma}[2.4) \\
\mu_{\left\{\nu \frac{a-1}{2} \rho\right\}}^{*}(\pi)=\left(\nu^{\frac{a-1}{2}} \rho\right)^{f} \otimes L\left(\Delta^{(5)}, \delta\left(\left[\nu^{\frac{-a+1}{2}} \rho, \nu^{\frac{a-3}{2}} \rho\right]\right)^{\ell_{\theta_{2}}} ; T_{\theta_{2}}\right),
\end{gathered}
$$

the result needed.

3.4. Calculating $\mu_{\left\{\nu^{x} \rho\right\}}^{*}(\pi)$ in the case $\pi \cong L\left(\left(\nu^{\frac{-a+1}{2}} \rho\right)^{k}, \delta\left(\left[\nu^{\frac{-a+1}{2}} \rho, \nu^{\frac{a-3}{2}} \rho\right]\right)^{\ell} ; T\right)$. Here, we address the problem of calculating $\mu_{\left\{\nu \frac{a-1}{2} \rho\right\}}^{*}(\pi)$ when

$$
\left.\pi \cong L\left(\nu^{\frac{-a+1}{2}} \rho\right)^{k}, \delta\left(\left[\nu^{\frac{-a+1}{2}} \rho, \nu^{\frac{a-3}{2}} \rho\right]\right)^{\ell} ; T\right) .
$$

We focus on the case when $\frac{a-1}{2} \equiv \operatorname{red}(\rho ; \sigma) \bmod 1$. We start by noting a couple of special cases where we have explicit formulae (and which do not work in the general algorithm given later). We have not included the case $a=3$ as it arises only for the integral case (the one not addressed in this paper). However, the case $a=3$ when $\operatorname{red}(\rho ; \sigma) \equiv \frac{1}{2} \bmod 1$ is discussed in section 8

Case $1(a=2)$. Here, we have the following results, proved in section 6.1 
Theorem 3.3. Let $\pi=L\left(\left(\nu^{-\frac{1}{2}} \rho\right)^{k} ; T\right)$. Suppose $\operatorname{red}(\rho ; \sigma) \equiv \frac{1}{2} \bmod 1$ and set $m=m_{T}(\rho, 2)$.

(1) If $(\rho, 2) \notin \operatorname{Jord}(T)$, then $f_{\pi}\left(\nu^{\frac{1}{2}} \rho\right)=0$.

(2) If (a) $\varepsilon(\rho, 2)=1, k$ even, $m$ odd, or (b) $\varepsilon(\rho, 2)=-1, k$ odd, $m$ odd,

$$
\mu_{\left\{\nu^{\frac{1}{2}} \rho\right\}}^{*}(\pi)=\left(\nu^{\frac{1}{2}} \rho\right)^{m} \otimes L\left(\left(\nu^{-\frac{1}{2}} \rho\right)^{k-1} ; T^{\prime}\right) .
$$

(3) If (a) $\varepsilon(\rho, 2)=1, k$ even, $m$ even, or $(\mathrm{b}) \varepsilon(\rho, 2)=-1, k$ odd, $m$ even,

$$
\mu_{\left\{\nu^{\frac{1}{2}} \rho\right\}}^{*}(\pi)=\left(\nu^{\frac{1}{2}} \rho\right)^{m} \otimes L\left(\left(\nu^{-\frac{1}{2}} \rho\right)^{k} ; T^{\prime \prime}\right) .
$$

(4) If (a) $\varepsilon(\rho, 2)=1, k$ odd, $m$ odd or (b) $\varepsilon(\rho, 2)=-1, k$ even, $m$ odd,

$$
\mu_{\left\{\nu^{\frac{1}{2}} \rho\right\}}^{*}(\pi)=\left(\nu^{\frac{1}{2}} \rho\right)^{m-1} \otimes L\left(\left(\nu^{-\frac{1}{2}} \rho\right)^{k} ; T^{\prime}\right) .
$$

(5) If (a) $\varepsilon(\rho, 2)=1, k$ odd, $m$ even, or (b) $\varepsilon(\rho, 2)=-1, k$ even, $m$ even,

$$
\mu_{\left\{\nu^{\frac{1}{2}} \rho\right\}}^{*}(\pi)=\left(\nu^{\frac{1}{2}} \rho\right)^{m-1} \otimes L\left(\left(\nu^{-\frac{1}{2}} \rho\right)^{k+1} ; T^{\prime \prime}\right) .
$$

Here, $m_{T^{\prime}}(\rho, 2)=1, m_{T^{\prime \prime}}(\rho, 2)=0$, the remaining multiplicities for $T^{\prime}, T^{\prime \prime}$ match those for $T, \varepsilon_{T^{\prime}}=\varepsilon_{T}$, and $\varepsilon_{T^{\prime \prime}}$ is the restriction of $\varepsilon_{T}$.

Case $2\left(a>3\right.$ and $\left.f_{\pi}\left(\nu^{\frac{a-3}{2}} \rho\right)=0\right)$. In this case, we have the following result, proved in section 6.2

Proposition 3.4. Let $\pi \cong L\left(\left(\nu^{\frac{-a+1}{2}} \rho\right)^{k}, \delta\left(\left[\nu^{\frac{-a+1}{2}} \rho, \nu^{\frac{a-3}{2}} \rho\right]\right)^{\ell} ; T\right)$ for some $a>3$ with $\frac{a-1}{2} \equiv \operatorname{red}(\rho ; \sigma) \bmod 1$. Suppose $f_{\pi}\left(\nu^{\frac{a-3}{2}} \rho\right)=0$. Then, we must have $\ell=0$ and either (i) $(\rho, a-2) \notin \operatorname{Jord}(T)$, or (ii) $(\rho, a-2),(\rho, a-4) \in \operatorname{Jord}(T)$ with $m_{T}(\rho, a-2)=1$ and $\varepsilon_{T}(\rho, a-2) \varepsilon_{T}(\rho, a-4)^{-1}=-1$ (interpreted as $\varepsilon_{T}(\rho, 2)=-1$ for $a=4)$. Further, we have the following:

(1) If $(\rho, a) \in \operatorname{Jord}(T)$ but $(\rho, a-2) \notin \operatorname{Jord}(T)$,

$$
\mu_{\left\{\nu \frac{a-1}{2} \rho\right\}}^{*}(\pi)=\left(\nu^{\frac{a-1}{2}} \rho\right)^{k+m_{T}(\rho, a)} \otimes T_{1},
$$

where $T_{1}$ has $m_{T_{1}}(\rho, a-2)=m_{T}(\rho, a), m_{T_{1}}(\rho, a)=0$, the remaining multiplicities match those of $T$, and $\varepsilon_{T_{1}}$ is given by substituting $(\rho, a-2)$ for $(\rho, a)$. More precisely, $\varepsilon_{T_{1}}$ is determined by the following changes: $\varepsilon_{T_{1}}(\rho, a-2)=\varepsilon_{T}(\rho, a)$ if defined, and $\varepsilon_{T_{1}}(\rho, a-2) \varepsilon_{T_{1}}(\rho, b)^{-1}=$ $\varepsilon_{T}(\rho, a) \varepsilon_{T}(\rho, b)^{-1}$.

(2) If $(\rho, a),(\rho, a-2) \in \operatorname{Jord}(T), \varepsilon_{T}(\rho, a) \varepsilon_{T}(\rho, a-2)^{-1}=-1$, and $m_{T}(\rho, a)$ odd,

$$
\mu_{\left\{\nu \frac{a-1}{2} \rho\right\}}^{*}(\pi)=\left(\nu^{\frac{a-1}{2}} \rho\right)^{k+m_{T}(\rho, a)-1} \otimes T_{2},
$$

where $T_{2}$ has $m_{T_{2}}(\rho, a-2)=m_{T}(\rho, a), m_{T_{2}}(\rho, a)=1$, and remaining data matching that of $T$.

(3) If $(\rho, a),(\rho, a-2) \in \operatorname{Jord}(T), \varepsilon_{T}(\rho, a) \varepsilon_{T}(\rho, a-2)^{-1}=-1$, and $m_{T}(\rho, a)$ even,

$$
\mu_{\left\{\nu \frac{a-1}{2} \rho\right\}}^{*}(\pi)=\left(\nu^{\frac{a-1}{2}} \rho\right)^{k+m_{T}(\rho, a)-1} \otimes L\left(\delta\left(\left[\nu^{\frac{-a+1}{2}} \rho, \nu^{\frac{a-3}{2}} \rho\right]\right) ; T_{3}\right),
$$

where $T_{3}$ has $m_{T_{3}}(\rho, a-2)=m_{T}(\rho, a)-1, m_{T_{3}}(\rho, a)=0$, the remaining multiplicities for $T_{3}$ match those for $T$, and $\varepsilon_{T_{3}}$ is the restriction of $\varepsilon_{T}$. 
(4) If $(\rho, a),(\rho, a-2) \in \operatorname{Jord}(T)$ and $\varepsilon_{T}(\rho, a) \varepsilon_{T}(\rho, a-2)^{-1}=1$,

$$
\mu_{\left\{\nu \frac{a-1}{2} \rho\right\}}^{*}(\pi)=\left(\nu^{\frac{a-1}{2}} \rho\right)^{k+m_{T}(\rho, a)-1} \otimes L\left(\nu^{\frac{-a+1}{2}} \rho ; T_{4}\right),
$$

where $T_{4}$ has $m_{T_{4}}(\rho, a-2)=m_{T}(\rho, a)+1, m_{T_{4}}(\rho, a)=0$, the remaining multiplicities for $T_{4}$ match those for $T$, and $\varepsilon_{T_{4}}$ is the restriction of $\varepsilon_{T}$.

(5) If $(\rho, a),(\rho, a-2) \notin \operatorname{Jord}(T)$, then

$$
\mu_{\left\{\nu \frac{a-1}{2} \rho\right\}}^{*}(\pi)=\left(\nu^{\frac{a-1}{2}} \rho\right)^{k} \otimes T .
$$

(6) If $(\rho, a) \notin \operatorname{Jord}(T)$ but $(\rho, a-2) \in \operatorname{Jord}(T)$, then

$$
\mu_{\left\{\nu \frac{a-1}{2} \rho\right\}}^{*}(\pi)=\left(\nu^{\frac{a-1}{2}} \rho\right)^{k-1} \otimes L\left(\nu^{\frac{-a+1}{2}} \rho ; T\right) .
$$

Case $3\left(a>3\right.$ and $\left.f_{\pi}\left(\nu^{\frac{a-3}{2}} \rho\right)>0\right)$. Note that we retain the assumption $\frac{a-1}{2} \equiv$ $\operatorname{red}(\rho ; \sigma) \bmod 1$.

Let $X=\left\{\nu^{\frac{a-3}{2}} \rho, \nu^{\frac{a-1}{2}} \rho\right\}$. The process in this case involves working through $\mu_{X}^{*}$ (noting that as $a>3, X$ satisfies the hypotheses for (1.2)). Thus we begin with a couple of general observations regarding $\mu_{X}^{*}$.

The following is just a restatement of Lemma 1.3.5 of [J5].

Lemma 3.5. An irreducible representation of a general linear group with support on $X$ has one of the following forms:

$$
\begin{aligned}
& \left(\nu^{\frac{a-1}{2}} \rho\right)^{x} \times \zeta\left(\left[\nu^{\frac{a-3}{2}} \rho, \nu^{\frac{a-1}{2}} \rho\right]\right)^{y} \times \delta\left(\left[\nu^{\frac{a-3}{2}} \rho, \nu^{\frac{a-1}{2}} \rho\right]\right)^{z} \\
& \left(\nu^{\frac{a-3}{2}} \rho\right)^{x} \times \zeta\left(\left[\nu^{\frac{a-3}{2}} \rho, \nu^{\frac{a-1}{2}} \rho\right]\right)^{y} \times \delta\left(\left[\nu^{\frac{a-3}{2}} \rho, \nu^{\frac{a-1}{2}} \rho\right]\right)^{z} .
\end{aligned}
$$

As a consequence, we have a couple of ways to calculate $\mu_{X}^{*}(\pi), \pi$ an irreducible representation of a classical group (not necessarily $\pi \cong L\left(\nu^{-\frac{a+1}{2}} \rho\right)^{k}, \delta\left(\left[\nu^{-\frac{a+1}{2}} \rho\right.\right.$, $\left.\left.\left.\nu^{\frac{a-3}{2}} \rho\right]\right)^{\ell} ; T\right)$ for this part of the discussion). We could calculate

$$
\begin{aligned}
\mu_{\left\{\nu \frac{a-1}{2} \rho\right\}}^{*}(\pi) & =\left(\nu^{\frac{a-1}{2}} \rho\right)^{\alpha_{1}} \otimes \lambda_{1}, \\
\mu_{\left\{\nu \frac{a-3}{2} \rho\right\}}^{*}\left(\lambda_{1}\right) & =\left(\nu^{\frac{a-3}{2}} \rho\right)^{\alpha_{2}} \otimes \lambda_{2}, \\
\mu_{\left\{\nu \frac{a-1}{2} \rho\right\}}^{*}\left(\lambda_{2}\right) & =\left(\nu^{\frac{a-1}{2}} \rho\right)^{\alpha_{3}} \otimes \lambda_{3} .
\end{aligned}
$$

It follows from Lemma 3.5 that $\theta_{\pi}(X)=\lambda_{3}$ and

$$
\tau_{\pi}(X)=\left\{\begin{aligned}
\left(\nu^{\frac{a-1}{2}} \rho\right)^{\alpha_{1}+\alpha_{3}-\alpha_{2}} & \times \zeta\left(\left[\nu^{\frac{a-3}{2}} \rho, \nu^{\frac{a-1}{2}} \rho\right]\right)^{\alpha_{3}} \\
& \times \delta\left(\left[\nu^{\frac{a-3}{2}} \rho, \nu^{\frac{a-1}{2}} \rho\right]\right)^{\alpha_{2}-\alpha_{3}} \text { if } \alpha_{1}+\alpha_{3} \geq \alpha_{2}, \\
\left(\nu^{\frac{a-3}{2}} \rho\right)^{\alpha_{2}-\alpha_{1}-\alpha_{3}} & \times \zeta\left(\left[\nu^{\frac{a-3}{2}} \rho, \nu^{\frac{a-1}{2}} \rho\right]\right)^{\alpha_{3}} \\
& \times \delta\left(\left[\nu^{\frac{a-3}{2}} \rho, \nu^{\frac{a-1}{2}} \rho\right]\right)^{\alpha_{1}} \text { if } \alpha_{1}+\alpha_{3} \leq \alpha_{2}
\end{aligned}\right.
$$

(noting that in the first case, one necessarily has $\alpha_{2}-\alpha_{3} \geq 0$ ). One could also start with $\nu^{\frac{a-3}{2}} \rho$ :

$$
\begin{aligned}
& \mu_{\left\{\nu \frac{a-3}{2} \rho\right\}}^{*}(\pi)=\left(\nu^{\left.\frac{a-3}{2} \rho\right)^{\alpha_{1}^{\prime}} \otimes \lambda_{1}^{\prime},}\right. \\
& \mu_{\left\{\nu \frac{a-1}{2} \rho\right\}}^{*}\left(\lambda_{1}^{\prime}\right)=\left(\nu^{\frac{a-1}{2}} \rho\right)^{\alpha_{2}^{\prime}} \otimes \lambda_{2}^{\prime}, \\
& \mu_{\left\{\nu \frac{a-3}{2} \rho\right\}}^{*}\left(\lambda_{2}^{\prime}\right)=\left(\nu^{\frac{a-3}{2}} \rho\right)^{\alpha_{3}^{\prime} \otimes \lambda_{3}^{\prime} .}
\end{aligned}
$$


Again, $\theta_{\pi}(X)=\lambda_{3}^{\prime}$ and

$$
\tau_{\pi}(X)=\left\{\begin{aligned}
\left(\nu^{\frac{a-3}{2}} \rho\right)^{\alpha_{1}^{\prime}+\alpha_{3}^{\prime}-\alpha_{2}^{\prime}} & \times \zeta\left(\left[\nu^{\frac{a-3}{2}} \rho, \nu^{\frac{a-1}{2}} \rho\right]\right)^{\alpha_{2}^{\prime}-\alpha_{3}^{\prime}} \\
& \times \delta\left(\left[\nu^{\frac{a-3}{2}} \rho, \nu^{\frac{a-1}{2}} \rho\right]\right)^{\alpha_{3}^{\prime}} \text { if } \alpha_{1}^{\prime}+\alpha_{3}^{\prime} \geq \alpha_{2}^{\prime}, \\
\left(\nu^{\frac{a-1}{2}} \rho\right)^{\alpha_{2}^{\prime}-\alpha_{1}^{\prime}-\alpha_{3}^{\prime}} \times \zeta\left(\left[\nu^{\frac{a-3}{2}} \rho, \nu^{\frac{a-1}{2}} \rho\right]\right)^{\alpha_{1}^{\prime}} & \times \delta\left(\left[\nu^{\frac{a-3}{2}} \rho, \nu^{\frac{a-1}{2}} \rho\right]\right)^{\alpha_{3}^{\prime}} \text { if } \alpha_{1}^{\prime}+\alpha_{3}^{\prime} \leq \alpha_{2}^{\prime}
\end{aligned}\right.
$$

(noting that in the first case, one necessarily has $\alpha_{2}^{\prime}-\alpha_{3}^{\prime} \geq 0$ ).

Returning to $\left.\pi \cong L\left(\nu^{-\frac{a+1}{2}} \rho\right)^{k}, \delta\left(\left[\nu^{-\frac{a+1}{2}} \rho, \nu^{\frac{a-3}{2}} \rho\right]\right)^{\ell} ; T\right)$, although we do not have a way to calculate $\mu_{\left\{\nu \frac{a-1}{2}\right.}^{*}(\pi)$ at this point, we can determine $\mu_{\left\{\nu \frac{a-3}{2} \rho\right\}}^{*}(\pi)$ as this fits under the previous case (see section 3.3$)$. Further, as we have $f_{\pi}\left(\nu^{\frac{a-3}{2}} \rho\right)>0$, the resulting $\lambda_{1}^{\prime}$ is associated to a group of lower rank. By inductive hypothesis, we may then continue on and determine $\mu_{X}^{*}(\pi)$ as above (second approach, i.e., calculating $\mu_{X}^{*}$ working in the order $\left.\mu_{\left\{\nu \frac{a-3}{2} \rho\right\}}^{*}, \mu_{\left\{\nu \frac{a-1}{2} \rho\right\}}^{*}, \mu_{\left\{\nu \frac{a-3}{2} \rho\right\}}^{*}\right)$. As $\tau_{X}(\pi)$ determines $f_{\pi}\left(\nu^{\frac{a-1}{2}} \rho\right)$, we can determine whether or not $f_{\pi}\left(\nu^{\frac{a-1}{2}} \rho\right)=0$. Of course, if it is zero, there is nothing left to do.

If $f_{\pi}\left(\nu^{\frac{a-1}{2}} \rho\right)>0$, then we have $\theta_{\pi}(X)=\lambda_{3}=\lambda_{3}^{\prime}$. From $\tau_{\pi}(X)$, we may easily determine $\alpha_{1}, \alpha_{2}, \alpha_{3}$, noting $\alpha_{1}=f_{\pi}\left(\nu^{\frac{a-1}{2}} \rho\right)>0$. As we are dealing with lowerrank groups, we may then detemine $\lambda_{2}$ such that $\mu_{\left\{\nu^{\frac{a-1}{2}} \rho\right\}}^{*}\left(\lambda_{2}\right)=\left(\nu^{\frac{a-1}{2}} \rho\right)^{\alpha_{3}} \otimes \lambda_{3}$ and then $\lambda_{1}$ such that $\mu_{\left\{\nu \frac{a-3}{2} \rho\right\}}^{*}\left(\lambda_{1}\right)=\left(\nu^{\frac{a-3}{2}} \rho\right)^{\alpha_{2}} \otimes \lambda_{2}$. We then have $\theta_{\pi}\left(\nu^{\frac{a-1}{2}} \rho\right)=\lambda_{1}$, and have thus determined $\mu_{\left\{\nu^{\frac{a-1}{2}} \rho\right\}}^{*}(\pi)$, as needed.

\section{EXAmples}

In this section, we do a couple of examples to illustrate the algorithms from the previous section. The first is a duality calulation using the algorithm for calculating duality discussed in the introduction, implemented using the algorithms from sections 3.2 and 3.3 to do the needed Jacquet module calculations. An example which included a nontrivial use of the algorithm in section 3.4 would be considerably longer, so we provide a second example illustrating the use of that algorithm.

4.1. Example 1. Given $\pi$, specified by its Langlands data, we compute the Langlands data for $\hat{\pi}$.

Suppose $\operatorname{red}(\rho ; \sigma)=\frac{1}{2}$. Write

$$
\delta\left(\left[\nu^{-\frac{1}{2}} \rho, \nu^{\frac{1}{2}} \rho\right]\right) \rtimes \sigma=T_{+}\left(\delta\left(\left[\nu^{-\frac{1}{2}} \rho, \nu^{\frac{1}{2}} \rho\right]\right) ; \sigma\right) \oplus T_{-}\left(\delta\left(\left[\nu^{-\frac{1}{2}} \rho, \nu^{\frac{1}{2}} \rho\right]\right)\right),
$$

where the components have

$$
r_{M, G}\left(T_{+}\left(\delta\left(\left[\nu^{-\frac{1}{2}} \rho, \nu^{\frac{1}{2}} \rho\right]\right) ; \sigma\right)\right)=2 \cdot \nu^{\frac{1}{2}} \rho \otimes \nu^{\frac{1}{2}} \rho \otimes \sigma+\nu^{\frac{1}{2}} \rho \otimes \nu^{-\frac{1}{2}} \rho
$$

and

$$
r_{M, G}\left(T_{+}\left(\delta\left(\left[\nu^{-\frac{1}{2}} \rho, \nu^{\frac{1}{2}} \rho\right]\right) ; \sigma\right)\right)=\nu^{\frac{1}{2}} \rho \otimes \nu^{-\frac{1}{2}} \rho .
$$

In terms of data, $\operatorname{Jord}_{\rho}\left(T_{ \pm}\left(\delta\left(\left[\nu^{-\frac{1}{2}} \rho, \nu^{\frac{1}{2}} \rho\right]\right) ; \sigma\right)\right)=\{(\rho, 2)\}$ with $m_{T_{ \pm}}(\rho, 2)=2$ and $\varepsilon_{T_{ \pm}}(\rho, 2)= \pm 1$ (signs matching). Let

$$
\pi=L\left(\delta\left(\left[\nu^{-\frac{5}{2}} \rho, \nu^{\frac{1}{2}} \rho\right]\right), \nu^{-\frac{1}{2}} \rho ; T_{-}\left(\left(\delta\left(\left[\nu^{-\frac{1}{2}} \rho, \nu^{\frac{1}{2}} \rho\right]\right) ; \sigma\right) .\right.\right.
$$

In what follows, we show that

$$
\hat{\pi}=L\left(\nu^{-\frac{5}{2}} \rho, \delta\left(\left[\nu^{-\frac{3}{2}} \rho, \nu^{-\frac{1}{2}} \rho\right]\right),\left(\nu^{-\frac{1}{2}} \rho\right)^{2} ; T_{+}\left(\delta\left(\left[\nu^{-\frac{1}{2}} \rho, \nu^{\frac{1}{2}} \rho\right]\right) ; \sigma\right)\right) .
$$


Note that if we specialize to $\rho=1_{F^{\times}}, \sigma=1_{S O(1)}$, we have

$$
\pi=L\left(S t_{G L(4)}\left(\nu^{-1}\right), \nu^{-\frac{1}{2}}, T_{-}\right)
$$

and $\hat{\pi}=L\left(\nu^{-\frac{5}{2}}, S t_{G L(2)}\left(\nu^{-1}\right), \nu^{-\frac{1}{2}} \times \nu^{-\frac{1}{2}} ; T_{+}\right)($in $S O(15, F))$.

Note that in the beginning of the calculations below, we have favored calculating $\mu_{\left\{\nu^{x} \rho\right\}}^{*}$ for $x>0$ as this simplifies the later calculations. In particular, on the dual side, the resulting $\left(\nu^{-x} \rho\right) \otimes \ldots$ must be associated to (1) the context of (3.1), so we are essentially dealing with a general linear groups calculation at that point (see Note 3.2).

Step 1.

$$
\mu_{\left\{\nu^{\frac{5}{2}} \rho\right\}}^{*}(\pi)=\nu^{\frac{5}{2}} \rho \otimes L\left(\delta\left(\left[\nu^{-\frac{3}{2}} \rho, \nu^{\frac{1}{2}} \rho\right]\right), \nu^{-\frac{1}{2}} \rho ; T_{-}\left(\delta\left(\left[\nu^{-\frac{1}{2}} \rho, \nu^{\frac{1}{2}} \rho\right]\right) ; \sigma\right)\right)=\nu^{\frac{5}{2}} \rho \otimes \theta_{1}
$$

(defining $\theta_{1}$ ).

To see this using the algorithm in section 3.3 , we have

$$
\begin{aligned}
& \pi\left.\hookrightarrow \mathcal{L}\left(\delta\left(\left[\nu^{-\frac{5}{2}} \rho, \nu^{\frac{1}{2}} \rho\right]\right), \nu^{-\frac{1}{2}} \rho\right) \rtimes T_{-}\left(\delta\left(\left[\nu^{-\frac{1}{2}} \rho, \nu^{\frac{1}{2}} \rho\right]\right) ; \sigma\right)\right) \\
&\left.\hookrightarrow \mathcal{L}\left(\delta\left(\left[\nu^{-\frac{3}{2}} \rho, \nu^{\frac{1}{2}} \rho\right]\right), \nu^{-\frac{1}{2}} \rho\right) \times \nu^{-\frac{5}{2}} \rho \rtimes T_{-}\left(\delta\left(\left[\nu^{-\frac{1}{2}} \rho, \nu^{\frac{1}{2}} \rho\right]\right) ; \sigma\right)\right) \\
& \Downarrow(\text { Claim 1) } \\
&\left.\pi \hookrightarrow \mathcal{L}\left(\delta\left(\left[\nu^{-\frac{3}{2}} \rho, \nu^{\frac{1}{2}} \rho\right]\right), \nu^{-\frac{1}{2}} \rho\right) \rtimes L\left(\nu^{-\frac{5}{2}} \rho ; T_{-}\left(\delta\left(\left[\nu^{-\frac{1}{2}} \rho, \nu^{\frac{1}{2}} \rho\right]\right) ; \sigma\right)\right)\right) .
\end{aligned}
$$

By Proposition 3.4, we have

$$
\left.\left.\mu_{\left\{\nu^{\frac{5}{2}} \rho\right\}}^{*}\left(L\left(\nu^{-\frac{5}{2}} \rho ; T_{-}\left(\delta\left(\left[\nu^{-\frac{1}{2}} \rho, \nu^{\frac{1}{2}} \rho\right]\right) ; \sigma\right)\right)\right)=\nu^{\frac{5}{2}} \rho \otimes T_{-}\left(\delta\left(\left[\nu^{-\frac{1}{2}} \rho, \nu^{\frac{1}{2}} \rho\right]\right) ; \sigma\right)\right)\right) .
$$

Then,

$$
\begin{gathered}
\left.\pi \hookrightarrow \mathcal{L}\left(\delta\left(\left[\nu^{-\frac{3}{2}} \rho, \nu^{\frac{1}{2}} \rho\right]\right), \nu^{-\frac{1}{2}} \rho\right) \times \nu^{\frac{5}{2}} \rho \rtimes T_{-}\left(\delta\left(\left[\nu^{-\frac{1}{2}} \rho, \nu^{\frac{1}{2}} \rho\right]\right) ; \sigma\right)\right) \\
\Downarrow(\operatorname{Claim}[2) \\
\left.\pi \hookrightarrow \mathcal{L}\left(\delta\left(\left[\nu^{-\frac{3}{2}} \rho, \nu^{\frac{1}{2}} \rho\right]\right), \nu^{-\frac{1}{2}} \rho, \nu^{\frac{5}{2}} \rho\right) \rtimes T_{-}\left(\delta\left(\left[\nu^{-\frac{1}{2}} \rho, \nu^{\frac{1}{2}} \rho\right]\right) ; \sigma\right)\right) \\
\Downarrow \\
\left.\pi \hookrightarrow \nu^{\frac{5}{2}} \rho \times \mathcal{L}\left(\delta\left(\left[\nu^{-\frac{3}{2}} \rho, \nu^{\frac{1}{2}} \rho\right]\right), \nu^{-\frac{1}{2}} \rho\right) \rtimes T_{-}\left(\delta\left(\left[\nu^{-\frac{1}{2}} \rho, \nu^{\frac{1}{2}} \rho\right]\right) ; \sigma\right)\right) \\
\Downarrow \\
\theta_{1}=L\left(\delta\left(\left[\nu^{-\frac{3}{2}} \rho, \nu^{\frac{1}{2}} \rho\right]\right), \nu^{-\frac{1}{2}} \rho ; T_{-}\left(\delta\left(\left[\nu^{-\frac{1}{2}} \rho, \nu^{\frac{1}{2}} \rho\right]\right) ; \sigma\right)\right),
\end{gathered}
$$

as claimed.

Step 2.

$$
\mu_{\left\{\nu^{\frac{1}{2}} \rho\right\}}^{*}\left(\theta_{1}\right)=\left(\nu^{\frac{1}{2}} \rho\right)^{3} \otimes L\left(\delta\left(\left[\nu^{-\frac{3}{2}} \rho, \nu^{-\frac{1}{2}} \rho\right]\right), \nu^{-\frac{1}{2}} \rho ; \sigma\right)=\left(\nu^{\frac{1}{2}} \rho\right)^{3} \otimes \theta_{2}
$$

(defining $\theta_{2}$ ).

To see this using the results of section 3.3 , observe that

$$
\begin{aligned}
\theta_{1} \hookrightarrow \delta\left(\left[\nu^{-\frac{3}{2}} \rho, \nu^{\frac{1}{2}} \rho\right]\right) & \times \nu^{-\frac{1}{2}} \rho \rtimes T_{-}\left(\delta\left(\left[\nu^{-\frac{1}{2}} \rho, \nu^{\frac{1}{2}} \rho\right]\right) ; \sigma\right) \\
& \Downarrow(\text { Claim 1) } \\
\theta_{1} \hookrightarrow \delta\left(\left[\nu^{-\frac{3}{2}} \rho, \nu^{\frac{1}{2}} \rho\right]\right) & \rtimes L\left(\nu^{-\frac{1}{2}} \rho ; T_{-}\left(\delta\left(\left[\nu^{-\frac{1}{2}} \rho, \nu^{\frac{1}{2}} \rho\right]\right) ; \sigma\right) .\right.
\end{aligned}
$$


By Theorem 3.3, we then have

$$
\begin{gathered}
\theta_{1} \hookrightarrow \delta\left(\left[\nu^{-\frac{3}{2}} \rho, \nu^{\frac{1}{2}} \rho\right]\right) \times\left(\nu^{\frac{1}{2}} \rho\right)^{2} \rtimes L\left(\nu^{-\frac{1}{2}} \rho ; \sigma\right) \\
\Downarrow(\text { Claim } 2) \\
\theta_{1} \hookrightarrow \mathcal{L}\left(\delta\left(\left[\nu^{-\frac{3}{2}} \rho, \nu^{\frac{1}{2}} \rho\right]\right),\left(\nu^{\frac{1}{2}} \rho\right)^{2}\right) \rtimes L\left(\nu^{-\frac{1}{2}} \rho ; \sigma\right) \\
\Downarrow \\
\theta_{1} \hookrightarrow\left(\nu^{\frac{1}{2}} \rho\right)^{3} \times \delta\left(\left[\nu^{-\frac{3}{2}} \rho, \nu^{-\frac{1}{2}} \rho\right]\right) \rtimes L\left(\nu^{-\frac{1}{2}} \rho ; \sigma\right)
\end{gathered}
$$

from which the identity of $\theta_{2}$ follows.

Step 3.

$$
\mu_{\left\{\nu^{\frac{3}{2}} \rho\right\}}^{*}\left(\theta_{2}\right)=\nu^{\frac{3}{2}} \rho \otimes L\left(\left(\nu^{-\frac{1}{2}} \rho\right)^{2} ; \sigma\right)=\nu^{\frac{3}{2}} \rho \otimes \theta_{3}
$$

(defining $\theta_{3}$ ). This may be argued using the algorithm from section 3.3 as above, but simplifies considerably as

$\theta_{2} \hookrightarrow \nu^{-\frac{1}{2}} \rho \times \nu^{-\frac{1}{2}} \rho \times \nu^{-\frac{3}{2}} \rho \rtimes \sigma \cong \nu^{-\frac{1}{2}} \rho \times \nu^{-\frac{1}{2}} \rho \times \nu^{\frac{3}{2}} \rho \rtimes \sigma \cong \nu^{\frac{3}{2}} \rho \times \nu^{-\frac{1}{2}} \rho \times \nu^{-\frac{1}{2}} \rho \rtimes \sigma$ (by the irreducibility of $\nu^{-\frac{3}{2}} \rho \rtimes \sigma$ and $\nu^{-\frac{1}{2}} \rho \times \nu^{\frac{3}{2}} \rho$ ).

Step 4.

$$
\mu_{\left\{\nu^{-\frac{1}{2}} \rho\right\}}^{*}\left(\theta_{3}\right)=\left(\nu^{-\frac{1}{2}} \rho\right)^{2} \otimes \sigma .
$$

Step 5.

$$
\begin{gathered}
\mu_{\left\{\nu^{\frac{1}{2}} \rho\right\}}^{*}\left(\hat{\theta}_{3}\right)=\left(\nu^{\frac{1}{2}} \rho\right)^{2} \otimes \sigma \\
\Downarrow \\
\hat{\theta}_{3}=T_{+}\left(\delta\left(\left[\nu^{-\frac{1}{2}} \rho, \nu^{\frac{1}{2}} \rho\right]\right) ; \sigma\right) .
\end{gathered}
$$

Step 6.

(by Lemma 2.4).

$$
\begin{gathered}
\mu_{\left\{\nu^{-\frac{3}{2}} \rho\right\}}^{*}\left(\hat{\theta}_{2}\right)=\nu^{-\frac{3}{2}} \rho \otimes T_{+}\left(\delta\left(\left[\nu^{-\frac{1}{2}} \rho, \nu^{\frac{1}{2}} \rho\right]\right) ; \sigma\right) \\
\Downarrow \\
\hat{\theta}_{2}=L\left(\nu^{-\frac{3}{2}} \rho ; T_{+}\left(\delta\left(\left[\nu^{-\frac{1}{2}} \rho, \nu^{\frac{1}{2}} \rho\right]\right) ; \sigma\right)\right)
\end{gathered}
$$

Step 7.

$$
\begin{gathered}
\mu_{\left\{\nu^{-\frac{1}{2}} \rho\right\}}^{*}\left(\hat{\theta}_{1}\right)=\left(\nu^{-\frac{1}{2}} \rho\right)^{3} \otimes L\left(\nu^{-\frac{3}{2}} ; T_{+}\left(\delta\left(\left[\nu^{-\frac{1}{2}} \rho, \nu^{\frac{1}{2}} \rho\right]\right) ; \sigma\right)\right) \\
\Downarrow \\
\hat{\theta}_{1}=L\left(\delta\left(\left[\nu^{-\frac{3}{2}} \rho, \nu^{-\frac{1}{2}} \rho\right]\right),\left(\nu^{-\frac{1}{2}} \rho\right)^{2} ; T_{+}\left(\delta\left(\left[\nu^{-\frac{1}{2}} \rho, \nu^{\frac{1}{2}} \rho\right]\right) ; \sigma\right)\right) .
\end{gathered}
$$

Note that from Step 2 , we see that if $\hat{\theta}_{1}=L(\Delta ; \mathcal{T})$,

$$
\begin{gathered}
\mu_{\left\{\nu^{-\frac{1}{2}} \rho\right\}}^{*}(L(\Delta ; \mathcal{T}))=\left(\nu^{-\frac{1}{2}} \rho\right)^{3} \otimes L\left(\nu^{-\frac{3}{2}} ; T_{+}\left(\delta\left(\left[\nu^{-\frac{1}{2}} \rho, \nu^{\frac{1}{2}} \rho\right]\right) ; \sigma\right)\right. \\
\Downarrow \\
m_{\left\{\nu^{-\frac{1}{2}} \rho\right\}}^{*}(\mathcal{L}(\Delta))=\left(\nu^{-\frac{1}{2}} \rho\right)^{3} \otimes \nu^{-\frac{3}{2}} \rho .
\end{gathered}
$$

It now follows from J55 that $\mathcal{L}(\Delta)=\mathcal{L}\left(\delta\left(\left[\nu^{-\frac{3}{2}} \rho, \nu^{-\frac{1}{2}} \rho\right]\right),\left(\nu^{-\frac{1}{2}} \rho\right)^{2}\right)$. The identity of $\hat{\theta}_{1}$ is then immediate.

Step 8.

$$
\begin{gathered}
\mu_{\left\{\nu^{-\frac{5}{2}} \rho\right\}}^{*}(\hat{\pi})=\nu^{-\frac{5}{2}} \rho \otimes L\left(\delta\left(\left[\nu^{-\frac{3}{2}} \rho, \nu^{-\frac{1}{2}} \rho\right]\right),\left(\nu^{-\frac{1}{2}} \rho\right)^{2} ; T_{+}\left(\delta\left(\left[\nu^{-\frac{1}{2}} \rho, \nu^{\frac{1}{2}} \rho\right]\right) ; \sigma\right)\right) \\
\Downarrow \\
\hat{\pi}=L\left(\nu^{-\frac{5}{2}} \rho, \delta\left(\left[\nu^{-\frac{3}{2}} \rho, \nu^{-\frac{1}{2}} \rho\right]\right),\left(\nu^{-\frac{1}{2}} \rho\right)^{2} ; T_{+}\left(\delta\left(\left[\nu^{-\frac{1}{2}} \rho, \nu^{\frac{1}{2}} \rho\right]\right) ; \sigma\right)\right)
\end{gathered}
$$

as in Step 7. 
4.2. Example 2. Given $\pi$ as in Case 3 of section 3.4, we compute $\mu_{\left\{\nu \frac{a-1}{2} \rho\right\}}^{*}(\pi)$.

Again, suppose $\operatorname{red}(\rho ; \sigma)=\frac{1}{2}$. Let $T \leq \delta\left(\left[\nu^{-\frac{1}{2}} \rho, \nu^{\frac{1}{2}} \rho\right]\right) \rtimes \delta\left(\left[\nu^{\frac{1}{2}} \rho, \nu^{\frac{3}{2}} \rho\right] ; \sigma\right)$ - the latter the generalized Steinberg representation whose minimal Jacquet module is $\nu^{\frac{3}{2}} \rho \otimes \nu^{\frac{1}{2}} \rho \otimes \sigma$-and having $\varepsilon_{T}(\rho, 2)=-1$. In particular, $\operatorname{Jord}_{\rho}(T)=\{(\rho, 2),(\rho, 4)\}$ with $m_{T}(\rho, 2)=2, m_{T}(\rho, 4)=1, \varepsilon_{T}(\rho, 2)=-1$, and $\varepsilon_{T}(\rho, 4)=1$. We let $\pi=$ $L\left(\left(\nu^{-\frac{3}{2}} \rho\right)^{3}, \delta\left(\left[\nu^{-\frac{3}{2}}, \nu^{\frac{1}{2}} \rho\right]\right) ; T\right)$ and calculate $\mu_{\left\{\nu^{\frac{3}{2}} \rho\right\}}^{*}(\pi)$.

Observe that we are in Case 3 from section 3.4 thus wish to calculate $\mu_{X}^{*}(\pi)$ for $X=\left\{\nu^{\frac{1}{2}} \rho, \nu^{\frac{3}{2}} \rho\right\}$. Using the notation from that section, we have

$$
\mu_{\left\{\nu^{\frac{1}{2}} \rho\right\}}^{*}(\pi)=\left(\nu^{\frac{1}{2}} \rho\right)^{2} \otimes \underbrace{L\left(\left(\nu^{-\frac{3}{2}} \rho\right)^{3}, \delta\left(\left[\nu^{-\frac{3}{2}} \rho, \nu^{-\frac{1}{2}} \rho\right]\right), \nu^{-\frac{1}{2}} \rho ; \delta\left(\left[\nu^{\frac{1}{2}} \rho, \nu^{\frac{3}{2}} \rho\right] ; \sigma\right)\right)}_{\lambda_{1}^{\prime}} .
$$

Next,

$$
\mu_{\left\{\nu^{\frac{3}{2}} \rho\right\}}^{*}\left(\lambda_{1}^{\prime}\right)=\left(\nu^{\frac{3}{2}} \rho\right)^{4} \otimes \underbrace{L\left(\nu^{-\frac{3}{2}} \rho,\left(\nu^{-\frac{1}{2}} \rho\right)^{2} ; \delta\left(\nu^{\frac{1}{2}} \rho ; \sigma\right)\right)}_{\lambda_{2}^{\prime}}
$$

and

$$
\mu_{\left\{\nu^{\frac{1}{2}} \rho\right\}}^{*}\left(\lambda_{2}^{\prime}\right)=\nu^{\frac{1}{2}} \rho \otimes L\left(\nu^{-\frac{3}{2}} \rho, \nu^{-\frac{1}{2}} \rho ; \delta\left(\nu^{\frac{1}{2}} \rho ; \sigma\right)\right) .
$$

It then follows that

$$
\mu_{X}^{*}(\pi)=\mathcal{L}\left(\left(\nu^{\frac{1}{2}} \rho\right)^{2}, \delta\left(\left[\nu^{\frac{1}{2}} \rho, \nu^{\frac{3}{2}} \rho\right]\right),\left(\nu^{\frac{3}{2}} \rho\right)^{3}\right) \otimes L\left(\nu^{-\frac{3}{2}} \rho, \nu^{-\frac{1}{2}} \rho ; \delta\left(\nu^{\frac{1}{2}} \rho ; \sigma\right)\right),
$$

noting that $\mathcal{L}\left(\left(\nu^{\frac{1}{2}} \rho\right)^{2}, \delta\left(\left[\nu^{\frac{1}{2}} \rho, \nu^{\frac{3}{2}} \rho\right]\right),\left(\nu^{\frac{3}{2}} \rho\right)^{3}\right)=\zeta\left(\left[\nu^{\frac{1}{2}} \rho, \nu^{\frac{3}{2}} \rho\right]\right)^{2} \times \delta\left(\left[\nu^{\frac{1}{2}} \rho, \nu^{\frac{3}{2}} \rho\right]\right) \times \nu^{\frac{3}{2}} \rho$ (irreducibly induced).

If we approach $\mu_{X}^{*}(\pi)$ in the other order, we may write

$$
\begin{aligned}
\mu_{\left\{\nu^{\frac{3}{2}} \rho\right\}}^{*}(\pi) & =\left(\nu^{\frac{3}{2}} \rho\right)^{2} \otimes \lambda_{1}, \\
\mu_{\left\{\nu^{\frac{1}{2}} \rho\right\}}^{*}\left(\lambda_{1}\right) & =\left(\nu^{\frac{1}{2}} \rho\right)^{3} \otimes \lambda_{2}, \\
\mu_{\left\{\nu^{\frac{3}{2}} \rho\right\}}^{*}\left(\lambda_{2}\right) & =\left(\nu^{\frac{3}{2}} \rho\right)^{2} \otimes L\left(\nu^{-\frac{3}{2}} \rho, \nu^{-\frac{1}{2}} \rho ; \delta\left(\nu^{\frac{1}{2}} \rho ; \sigma\right)\right) .
\end{aligned}
$$

Now,

$$
\lambda_{2}=L\left(\left(\nu^{-\frac{3}{2}} \rho\right)^{2}, \nu^{-\frac{1}{2}} \rho ; \delta\left(\left[\nu^{\frac{1}{2}} \rho, \nu^{\frac{3}{2}} \rho\right] ; \sigma\right)\right) .
$$

Then,

$$
\begin{gathered}
\mu_{\left\{\nu^{\frac{1}{2}} \rho\right\}}^{*}\left(\lambda_{1}\right)=\left(\nu^{\frac{1}{2}} \rho\right)^{3} \otimes L\left(\left(\nu^{-\frac{3}{2}} \rho\right)^{2}, \nu^{-\frac{1}{2}} \rho ; \delta\left(\left[\nu^{\frac{1}{2}} \rho, \nu^{\frac{3}{2}} \rho\right] ; \sigma\right)\right) \\
\Downarrow \\
\lambda_{1}=L\left(\left(\nu^{-\frac{3}{2}} \rho\right)^{2}, \mathcal{T}\right),
\end{gathered}
$$

where $\mathcal{T}=\delta\left(\left[\nu^{-\frac{1}{2}} \rho, \nu^{\frac{1}{2}} \rho\right]\right) \rtimes T$ (so $m_{\mathcal{T}}(\rho, 2)=4$, with the rest of the data matching that for $T)$.

\section{Proofs For SECTION 3.3}

In the algorithm given in section 3.3 , there are four claims made whose proofs are deferred to this section. In particular, claims 1-4 of section 3.3 are proven in Lemmas 5.4, 5.5, 5.6, and 5.7, respectively.

The following is Proposition 3.1 of $\mathrm{J} 8$. 
Proposition 5.1. Suppose $\pi \leq\left(\nu^{\frac{-a+1}{2}} \rho\right)^{k} \times \delta\left(\left[\nu^{\frac{-a+1}{2}} \rho, \nu^{\frac{a-3}{2}} \rho\right]\right)^{\ell} \rtimes T$ is irreducible. Then, $\pi$ has the form

$$
\pi=L\left(\left(\nu^{\frac{-a+1}{2}} \rho\right)^{k^{\prime}}, \delta\left(\left[\nu^{\frac{-a+1}{2}} \rho, \nu^{\frac{a-3}{2}} \rho\right]\right)^{\ell^{\prime}} ; T^{\prime}\right)
$$

with $k^{\prime}+\ell^{\prime} \leq k+\ell$.

Corollary 5.2. If $\pi=L\left(\left(\nu^{\frac{-a+1}{2}} \rho\right)^{k}, \delta\left(\left[\nu^{\frac{-a+1}{2}} \rho, \nu^{\frac{a-3}{2}} \rho\right]\right)^{\ell} ; T\right)$, then $\theta_{\pi}\left(\nu^{\frac{a-1}{2}} \rho\right)$ has the form

$$
\theta_{\pi}\left(\nu^{\frac{a-1}{2}} \rho\right)=L\left(\left(\nu^{\frac{-a+1}{2}} \rho\right)^{k^{\prime \prime}}, \delta\left(\left[\nu^{\frac{-a+1}{2}} \rho, \nu^{\frac{a-3}{2}} \rho\right]\right)^{\ell^{\prime \prime}} ; T^{\prime \prime}\right) .
$$

Proof. Since $\pi \leq\left(\nu^{\frac{-a+1}{2}} \rho\right)^{k} \times \delta\left(\left[\nu^{\frac{-a+1}{2}} \rho, \nu^{\frac{a-3}{2}} \rho\right]\right)^{\ell} \rtimes T$, we have

$$
\begin{aligned}
\mu^{*}(\pi) \leq & M \sum_{\substack{x_{1}, x_{2}, i_{1}, \ldots, i_{\ell} \\
j_{1}, \ldots j_{\ell}, h}}\left(\nu^{\frac{-a+1}{2}} \rho\right)^{x_{1}} \times\left(\nu^{\frac{a-1}{2}} \rho\right)^{x_{2}} \\
& \times\left(\prod_{s=1}^{\ell} \delta\left(\left[\nu^{-i_{s}+1} \rho, \nu^{\frac{a-3}{2}} \rho\right]\right) \times \delta\left(\left[\nu^{j_{s}} \rho, \nu^{\frac{a-1}{2}} \rho\right]\right)\right) \times \lambda_{h} \\
& \quad\left(\nu^{\frac{-a+1}{2}} \rho\right)^{k-x_{1}-x_{2}} \times\left(\prod_{s=1}^{\ell} \delta\left(\left[\nu^{i_{s}} \rho, \nu^{j_{s}-1} \rho\right]\right)\right) \rtimes \theta_{h}
\end{aligned}
$$

for some $M$. Now, observe that to contribute to $\mu_{\left\{\nu^{\frac{a-1}{2}} \rho\right\}}^{*}$, we must have $x_{1}=0$ and $-i_{s}+1=\frac{a-1}{2}$ for all $s$. Further, each $j_{s}$ must be either $\frac{a-1}{2}$ or $\frac{a+1}{2}$. Let $\ell_{1}$ denote the number of $s$ for which $j_{s}=\frac{a-1}{2}$. We then have

$$
\begin{aligned}
\mu_{\left\{\nu \frac{a-1}{2} \rho\right\}}^{*}(\pi) & \leq \sum\left(\nu^{\frac{a-1}{2}} \rho\right)^{x_{2}+\ell_{1}} \times \lambda_{h} \\
& \otimes\left(\nu^{\frac{-a+1}{2}} \rho\right)^{k-x_{2}} \times \delta\left(\left[\nu^{\frac{-a+3}{2}} \rho, \nu^{\frac{a-3}{2}} \rho\right]\right)^{\ell_{1}} \times \delta\left(\left[\nu^{\frac{-a+3}{2}} \rho, \nu^{\frac{a-1}{2}} \rho\right]\right)^{\ell-\ell_{1}} \rtimes \theta_{h} .
\end{aligned}
$$

Now, writing $\mu_{\left\{\nu \frac{a-1}{2} \rho\right\}}^{*}(T)=\left(\nu^{\frac{a-1}{2}} \rho\right)^{f_{T}} \otimes \theta_{T}$, it follows from Lemma 2.2 that

$$
\begin{aligned}
\theta_{\pi}\left(\nu^{\frac{a-1}{2}} \rho\right) \leq\left(\nu^{\frac{-a+1}{2}} \rho\right)^{k-x_{2}} & \times \delta\left(\left[\nu^{\frac{-a+3}{2}} \rho, \nu^{\frac{a-3}{2}} \rho\right]\right)^{\ell_{1}} \\
& \times \delta\left(\left[\nu^{\frac{-a+3}{2}} \rho, \nu^{\frac{a-1}{2}} \rho\right]\right)^{\ell-\ell_{1}} \rtimes\left(\left(\nu^{\frac{a-1}{2}} \rho\right)^{f_{T}+x_{2}+\ell_{1}-f_{\pi}} \rtimes \theta_{T}\right) .
\end{aligned}
$$

If $\theta_{T}=T^{*}$ is tempered, this gives

$$
\begin{aligned}
\theta_{\pi}\left(\nu^{\frac{a-1}{2}} \rho\right) \leq\left(\nu^{\frac{-a+1}{2}} \rho\right)^{k+f_{T}+\ell_{1}-f_{\pi}} & \times \delta\left(\left[\nu^{\frac{-a+1}{2}} \rho, \nu^{\frac{a-3}{2}} \rho\right]\right)^{\ell-\ell_{1}} \\
& \rtimes\left(\delta\left(\left[\nu^{\frac{-a+3}{2}} \rho, \nu^{\frac{a-3}{2}} \rho\right]\right)^{\ell_{1}} \rtimes T^{*}\right) ;
\end{aligned}
$$

if not, $\theta_{T}$ has the form $\theta_{T}=L\left(\left(\delta\left(\left[\nu^{\frac{-a+1}{2}} \rho, \nu^{\frac{a-3}{2}} \rho\right]\right) ; T^{* *}\right)\right.$ and

$$
\begin{aligned}
\theta_{\pi}\left(\nu^{\frac{a-1}{2}} \rho\right) \leq\left(\nu^{\frac{-a+1}{2}} \rho\right)^{k+f_{T}+\ell_{1}-f_{\pi}} & \times \delta\left(\left[\nu^{\frac{-a+1}{2}} \rho, \nu^{\frac{a-3}{2}} \rho\right]\right)^{\ell-\ell_{1}+1} \\
& \rtimes\left(\delta\left(\left[\nu^{\frac{-a+3}{2}} \rho, \nu^{\frac{a-3}{2}} \rho\right]\right)^{\ell_{1}} \rtimes T^{* *}\right) .
\end{aligned}
$$

In either case, it follows from Proposition 5.1 that $\theta_{\pi}\left(\nu^{\frac{a-1}{2}} \rho\right)$ has the form claimed.

Lemma 5.3. Suppose $\Delta \otimes T$ satisfies the requirements to be Langlands data.The only terms $\lambda \otimes \theta \leq \mu^{*}(\mathcal{L}(\Delta) \rtimes T)$ having central character of $\lambda$ matching that of $\Delta$ is $\mathcal{L}(\Delta) \otimes T$, which appears with multiplicity one. 
Write $M^{*}(\mathcal{L}(\Delta))=\sum_{i} \lambda_{i}^{\prime} \otimes \check{\lambda}_{i}^{\prime \prime \prime} \otimes \lambda_{i}^{\prime \prime}$, noting that $\lambda_{i}^{\prime} \otimes \lambda_{i}^{\prime \prime} \otimes \lambda_{i}^{\prime \prime \prime} \leq r_{M, G}(\mathcal{L}(\Delta))$. If $\mu^{*}(T)=\sum_{j} \zeta_{j} \otimes \theta_{j}$, then by Theorem 2.1

$$
\mu^{*}(\mathcal{L}(\Delta) \rtimes T)=\sum_{i, j} \lambda_{i}^{\prime} \times \check{\lambda}_{i}^{\prime \prime \prime} \times \zeta_{j} \otimes \lambda_{i}^{\prime \prime} \rtimes \theta_{j} .
$$

We are interested in when $\mathcal{L}(\Delta) \leq \lambda_{i}^{\prime} \times \check{\lambda}_{i}^{\prime \prime \prime} \times \zeta_{j}$. Observe that as $r_{M, G}(\mathcal{L}(\Delta)) \geq$ $\lambda_{i}^{\prime} \otimes \lambda_{i}^{\prime \prime} \otimes \lambda_{i}^{\prime \prime \prime}$, we must have the central exponent for $\check{\lambda}_{i}^{\prime \prime \prime} \times \zeta_{j}$ matching that of $\lambda_{i}^{\prime \prime} \times \lambda_{i}^{\prime \prime \prime}$. Further, observe that as the segments appearing in $\lambda_{i}^{\prime \prime} \otimes \lambda_{i}^{\prime \prime \prime}$ are the lower parts of segments from $\Delta$-which have negative central exponent - the central exponent of $\check{\lambda}_{i}^{\prime \prime \prime} \times \zeta_{j}$ must be negative. However, if nontrivial, $\zeta_{j}$ must have nonnegative central exponent by the Casselman criterion. Similarly, if nontrivial, $\check{\lambda}_{i}^{\prime \prime \prime}$ must have positive central exponent as $\lambda_{i}^{\prime \prime \prime}$ has negative central exponent (as above, it is comprised of lower parts of segments with negative central exponents). Thus, $\lambda_{i}^{\prime \prime \prime}$ and $\zeta_{j}$ must be trivial, leaving $\lambda_{i}^{\prime}=\mathcal{L}(\Delta)$, hence $\theta_{j}=T$. That $\mathcal{L}(\Delta) \otimes T$ appears with multiplicity one follows from properties of the Langlands classification (e.g., [B-J3]).

We now turn to the task of verifying the claims.

Lemma 5.4. Claim 1 holds.

Proof. To see this, first write $\lambda=L\left(\Delta^{*} ; T^{*}\right)$. Then,

$$
\begin{gathered}
\pi \hookrightarrow \mathcal{L}\left(\Delta^{(2)}\right) \times \mathcal{L}\left(\Delta^{*}\right) \rtimes T^{*} \\
\Downarrow(\text { Lemma } 2.2) \\
\pi \hookrightarrow \mathcal{L}\left(\Delta^{* *}\right) \rtimes T^{*}
\end{gathered}
$$

for some irreducible $\mathcal{L}\left(\Delta^{* *}\right) \leq \mathcal{L}\left(\Delta^{(2)}\right) \times \mathcal{L}\left(\Delta^{*}\right)$. Now, observe that if $\Sigma^{\prime}$ and $\Sigma^{\prime \prime}$ are two linked segments with negative central exponents, then $\Delta^{\prime} \cup \Delta^{\prime \prime}$ and $\Delta^{\prime} \cap \Delta^{\prime \prime}$ also have negative central exponents. It then follows from Lemma 2.3 that $\Delta^{* *}$ has negative central exponents. Therefore, by the Langlands classification we must have $\pi=L\left(\Delta^{* *} ; T^{*}\right)$. In particular, this forces $\Delta^{* *}=\Delta$ and $T^{*}=T$; the former implying $\mathcal{L}(\Delta) \leq \mathcal{L}\left(\Delta^{(2)}\right) \times \mathcal{L}\left(\Delta^{*}\right)$. Observe that this implies $\Delta^{*}$ has the same central character as $\left(\nu^{\frac{-a+1}{2}} \rho\right)^{k} \times \delta\left(\left[\nu^{\frac{-a+1}{2}} \rho, \nu^{\frac{a-3}{2}} \rho\right]\right)^{\ell}$. By Lemma 5.3 applied to $\left(\nu^{\frac{-a+1}{2}} \rho\right)^{k} \times \delta\left(\left[\nu^{\frac{-a+1}{2}} \rho, \nu^{\frac{a-3}{2}} \rho\right]\right)^{\ell} \rtimes T$, it then follows that $\mathcal{L}\left(\Delta^{*}\right)=\left(\nu^{\frac{-a+1}{2}} \rho\right)^{k} \times$ $\delta\left(\left[\nu^{\frac{-a+1}{2}} \rho, \nu^{\frac{a-3}{2}} \rho\right]\right)^{\ell}$, as needed.

Lemma 5.5. Claim 2 holds.

Proof. First, we make an initial claim: an irreducible subquotient of $\mathcal{L}\left(\Delta^{(2)}\right) \times$ $\left(\nu^{\frac{a-1}{2}} \rho\right)^{f_{1}}$ has the form $\mathcal{L}\left(\Delta^{*},\left(\nu^{\frac{a-1}{2}} \rho\right)^{f_{1}-x}\right)$ for some $0 \leq x \leq f_{1}$ and $\Delta^{*}$ consisting of segments with negative central exponents. To show this, it suffices by Lemma 2.3 to show the following: if a (multi)set of segments $\Delta_{0}$ satisfies (1) $\Delta_{0}$ consists of segments with negative central exponents and copies of $\nu^{\frac{a-1}{2}} \rho$ (segments with a single element), and (2) $\delta\left(\left[\nu^{\frac{-a+1}{2}} \rho, \nu^{\frac{a-3}{2}} \rho\right]\right)$ is not in $\Delta_{0}$, then any $\Delta_{0}^{\prime}$ obtained by replacing a pair of segments $\Sigma, \Sigma^{\prime}$ with $\Sigma \cup \Sigma^{\prime}, \Sigma \cap \Sigma^{\prime}$ (i.e., as in Lemma 2.3) also satisfies (1) and (2). This may be seen by considering two cases: first when one of the segments is $\nu^{\frac{a-1}{2}} \rho$, then when neither is. If one of the segments is $\nu^{\frac{a-1}{2}} \rho$, then the other must have the form $\left[\nu^{\frac{-d_{i}+1}{2}} \rho, \nu^{\frac{a-3}{2}} \rho\right]$ in order for the segments to be linked. This gives $\emptyset$ and $\left[\nu^{\frac{-d_{i}+1}{2}} \rho, \nu^{\frac{a-1}{2}} \rho\right]$ as the intersection and union. Clearly, (2) holds. In order for (1) to fail, we would have to have $d_{i} \leq a$. However, since $d_{i} \geq c_{i}+2=a$, the only possibility is $\left[\nu^{\frac{-d_{i}+1}{2}} \rho, \nu^{\frac{c_{i}-1}{2}} \rho\right]=\left[\nu^{\frac{-a+1}{2}} \rho, \nu^{\frac{a-3}{2}} \rho\right]$-ruled 
out by (2). If $\nu^{\frac{a-1}{2}} \rho$ is not one of the segments, write them as $\left[\nu^{\frac{-d_{i}+1}{2}} \rho, \nu^{\frac{c_{i}-1}{2}} \rho\right]$ and $\left[\nu \frac{-d_{j}+1}{2} \rho, \nu \frac{c_{j}-1}{2} \rho\right]$; without loss of generality, we may assume $c_{j}>c_{i}$. For the segments to be linked, we must then have $d_{j}<d_{i}$ (also $c_{i} \geq-d_{j}$ ); for negative central exponents, $c_{i}<d_{i}$ and $c_{j}<d_{j}$. Taking the intersection and union, we get $\left[\nu^{\frac{-d_{j}+1}{2}} \rho, \nu^{\frac{c_{i}-1}{2}} \rho\right]$ and $\left[\nu^{\frac{-d_{i}+1}{2}} \rho, \nu^{\frac{c_{j}-1}{2}} \rho\right]$, respectively. We have $c_{j}<d_{j}<d_{i}$ and $c_{i}<c_{j}<d_{j}$, so (1) is still satisfied. For (2), observe that to obtain $\left[\nu^{\frac{-a+1}{2}} \rho, \nu^{\frac{a-3}{2}} \rho\right]$ as the intersection, we must have $c_{i}=a-2$ and $d_{j}=a$. However, $c_{i}<c_{j}<d_{j}$ then becomes $a-2<c_{j}<a$, a contradiction as $c_{j} \equiv a \bmod 2$. Similarly, for $\left[\nu^{\frac{-a+1}{2}} \rho, \nu^{\frac{a-3}{2}} \rho\right]$ to be the union, we would require $c_{j}=a-2$ and $d_{i}=a$. Then, $c_{j}<d_{j}<d_{i}$ gives the contradiction. The initial claim now follows.

Now, suppose (for an indirect argument) we had $\lambda^{\prime}=\mathcal{L}\left(\Delta^{*},\left(\nu^{\frac{a-1}{2}} \rho\right)^{f_{1}-x}\right)$ for some $x>0$. By Corollary [5.2, $\theta_{1}=L\left(\left(\nu^{\frac{-a+1}{2}} \rho\right)^{k_{1}}, \delta\left(\left[\nu^{\frac{-a+1}{2}} \rho, \nu^{\frac{a-3}{2}} \rho\right]\right)^{\ell_{1}} ; T_{1}\right)$. Then, by Lemma 2.2 .

$$
\pi \hookrightarrow \mathcal{L}\left(\Delta^{*}\right) \times\left(\nu^{\frac{a-1}{2}} \rho\right)^{f_{1}-x} \rtimes \theta_{1} \Rightarrow \pi \hookrightarrow \mathcal{L}\left(\Delta^{*}\right) \rtimes \theta_{2}
$$

for some irreducible $\theta_{2} \leq\left(\nu^{\frac{a-1}{2}} \rho\right)^{f_{1}-x} \rtimes \theta_{1}$. Write

$$
\theta_{2}=L\left(\left(\nu^{\frac{-a+1}{2}} \rho\right)^{k_{2}}, \delta\left(\left[\nu^{\frac{-a+1}{2}} \rho, \nu^{\frac{a-3}{2}} \rho\right]\right)^{\ell_{2}} ; T_{2}\right)
$$

by Proposition 5.1. Again by Lemma 2.2.

$$
\pi \hookrightarrow \mathcal{L}\left(\Delta^{*}\right) \times \mathcal{L}\left(\left(\nu^{\frac{-a+1}{2}} \rho\right)^{k_{2}}, \delta\left(\left[\nu^{\frac{-a+1}{2}} \rho, \nu^{\frac{a-3}{2}} \rho\right]\right)^{\ell_{2}}\right) \rtimes T_{2} \Rightarrow \pi \hookrightarrow \mathcal{L}\left(\Delta^{* *}\right) \rtimes T_{2}
$$

for some irreducible $\mathcal{L}\left(\Delta^{* *}\right) \leq \mathcal{L}\left(\Delta^{*}\right) \times \mathcal{L}\left(\left(\nu^{\frac{-a+1}{2}} \rho\right)^{k_{2}}, \delta\left(\left[\nu^{\frac{-a+1}{2}} \rho, \nu^{\frac{a-3}{2}} \rho\right]\right)^{\ell_{2}}\right)$. By Lemma 2.4, since the central exponents of segments in $\Delta^{*},\left(\nu^{\frac{-a+1}{2}} \rho\right)$, and $\delta\left(\left[\nu^{\frac{-a+1}{2}} \rho, \nu^{\frac{a-3}{2}} \rho\right]\right)$ are all negative, so are the central exponents of segments in $\Delta^{* *}$. It then follows from the Langlands classification that $\pi=L\left(\Delta^{* *} ; T_{2}\right)$. We next show this is not the case.

To see this, we focus on the number of segments having upper end with exponents at least $\frac{a-1}{2}$. Let $n_{\Delta}$ be the number of such segments in $\Delta$. Since $\Delta^{(2)}$ is obtained from $\Delta$ by removing segments $\delta\left(\left[\nu^{\frac{-a+1}{2}} \rho, \nu^{\frac{a-3}{2}} \rho\right]\right)$ and then taking $\left.{ }_{\left\{\nu \frac{-a+1}{2}\right.} \rho\right\} m^{*}$, we see that $n_{\Delta}=n_{\Delta^{(2)}}$. By assumption, $n_{\Delta^{*}}=n_{\Delta^{(2)}}+x=n_{\Delta}+x, x>0$. By Lemma 2.3 applied to $\mathcal{L}\left(\Delta^{* *}\right) \leq \mathcal{L}\left(\Delta^{*}\right) \times \mathcal{L}\left(\left(\nu^{\frac{-a+1}{2}} \rho\right)^{k_{2}}, \delta\left(\left[\nu^{\frac{-a+1}{2}} \rho, \nu^{\frac{a-3}{2}} \rho\right]\right)^{\ell_{2}}\right)$, we see that $n_{\Delta^{* *}}=n_{\Delta^{*}}=n_{\Delta}+x$. Thus, we cannot have $\Delta^{* *}=\Delta$, so cannot have $\pi=L(\Delta ; T)=L\left(\Delta^{* *} ; T_{1}^{*}\right)$. Thus, we must have $x=0$, and the lemma follows.

Lemma 5.6. Claim 3 holds.

Proof. We have $\theta_{\pi}\left(\nu^{\frac{a-1}{2}} \rho\right) \leq \mathcal{L}\left(\Delta^{(4)}\right) \rtimes \theta_{2}$ and by (1.2) (in particular, uniqueness of $\left.\theta_{\pi}\right)$,

$$
r_{M, G}\left(\theta_{\pi}\left(\nu^{\frac{a-1}{2}} \rho\right)\right) \geq \mathcal{L}\left(\Delta^{(4)}\right) \otimes\left(\nu^{\frac{a-1}{2}} \rho\right)^{f_{2}} \otimes \theta_{1} .
$$

If we knew $\mu_{\mathcal{L}\left(\Delta^{(4)}\right)}^{*}\left(\mathcal{L}\left(\Delta^{(4)}\right) \rtimes \theta_{2}\right)=\mathcal{L}\left(\Delta^{(4)}\right) \otimes \theta_{2}$, it would follow that

$$
\mu_{\mathcal{L}\left(\Delta^{(4)}\right)}^{*}\left(\theta_{\pi}\left(\nu^{\frac{a-1}{2}} \rho\right)\right)=\mathcal{L}\left(\Delta^{(4)}\right) \otimes \theta_{2} \Rightarrow \mu^{*}\left(\theta_{2}\right) \geq\left(\nu^{\frac{a-1}{2}} \rho\right)^{f_{2}} \otimes \theta_{1} .
$$

Since $\theta_{2} \leq\left(\nu^{\frac{a-1}{2}} \rho\right)^{f_{2}} \rtimes \theta_{1}$ and $f_{\theta_{1}}\left(\nu^{\frac{a-1}{2}} \rho\right)=0$, Claim 3 follows. Thus Claim 3 is reduced to showing the following: for any $\theta^{\prime} \leq\left(\nu^{\frac{a-1}{2}} \rho\right)^{f_{2}} \rtimes \theta_{1}$, we have

$$
\mu_{\mathcal{L}\left(\Delta^{(4)}\right)}^{*}\left(\mathcal{L}\left(\Delta^{(4)}\right) \rtimes \theta^{\prime}\right)=\mathcal{L}\left(\Delta^{(4)}\right) \otimes \theta^{\prime} .
$$


Write $\theta_{1}=L\left(\left(\nu^{\frac{-a+1}{2}} \rho\right)^{k^{*}}, \delta\left(\left[\nu^{\frac{-a+1}{2}} \rho, \nu^{\frac{a-3}{2}} \rho\right]\right)^{\ell^{*}} ; T^{*}\right)$ (Corollary [5.2). Then

$$
\begin{aligned}
\mathcal{L}\left(\Delta^{(4)}\right) & \times\left(\nu^{\frac{a-1}{2}} \rho\right)^{f_{2}} \rtimes \theta_{1} \leq \mathcal{L}\left(\Delta^{(4)}\right) \\
& \times\left(\nu^{\frac{a-1}{2}} \rho\right)^{f_{2}} \times\left(\nu^{\frac{-a+1}{2}} \rho\right)^{k^{*}} \times \delta\left(\left[\nu^{\frac{-a+1}{2}} \rho, \nu^{\frac{a-3}{2}} \rho\right]\right)^{\ell^{*}} \rtimes T^{*} .
\end{aligned}
$$

Thus (5.1) would follow if we show

$$
\begin{array}{r}
\mu_{\mathcal{L}\left(\Delta^{(4)}\right)}^{*}\left(\mathcal{L}\left(\Delta^{(4)}\right) \times\left(\nu^{\frac{a-1}{2}} \rho\right)^{f_{2}} \times\left(\nu^{\frac{-a+1}{2}} \rho\right)^{k^{*}} \times \delta\left(\left[\nu^{\frac{-a+1}{2}} \rho, \nu^{\frac{a-3}{2}} \rho\right]\right)^{\ell^{*}} \rtimes T^{*}\right) \\
=\mathcal{L}\left(\Delta^{(4)}\right) \otimes\left(\left(\nu^{\frac{a-1}{2}} \rho\right)^{f_{2}} \times\left(\nu^{\frac{-a+1}{2}} \rho\right)^{k^{*}} \times \delta\left(\left[\nu^{\frac{-a+1}{2}} \rho, \nu^{\frac{a-3}{2}} \rho\right]\right)^{\ell^{*}} \rtimes T^{*}\right) .
\end{array}
$$

We use Tadić's $\mu^{*}$ structure to argue this. First, write $M^{*}\left(\mathcal{L}\left(\Delta^{(4)}\right)\right)=\sum_{i} \xi_{i}^{\prime} \otimes$ $\check{\xi}_{i}^{\prime \prime \prime} \otimes \xi_{i}^{\prime \prime}$. Note that $\xi_{i}^{\prime} \otimes \xi_{i}^{\prime \prime} \otimes \xi_{i}^{\prime \prime \prime} \leq r_{M, G}\left(\mathcal{L}\left(\Delta^{(4)}\right)\right.$. In particular, we have $m^{*}\left(\mathcal{L}\left(\Delta^{(4)}\right)\right)$ $\geq \mathcal{L}\left(\Delta_{i, 1}^{(4)}\right) \otimes \mathcal{L}\left(\Delta_{i, 2}^{(4)}\right)$ with $\mathcal{L}\left(\Delta_{i, 1}^{(4)}\right)=\xi_{i}^{\prime}$ and $m^{*}\left(\mathcal{L}\left(\Delta_{i, 2}^{(4)}\right)\right) \geq \xi_{i}^{\prime \prime} \otimes \xi_{i}^{\prime \prime \prime}$. Note the generalized Steinberg representations appearing in $\Delta_{i, 2}^{(4)}$ all have negative central exponents (as $\Delta_{i, 2}^{(4)}$ consists of the lower parts of segments in $\Delta^{(4)}$ and the generalized Steinberg representations in $\Delta^{(4)}$ all have negative central exponents). Also, observe that as $g_{\mathcal{L}\left(\Delta^{(4)}\right)}\left(\nu^{\frac{-a+1}{2}} \rho\right)=0$, we must also have $g_{\mathcal{L}\left(\Delta_{i, 2}^{(4)}\right)}\left(\nu^{\frac{-a+1}{2}} \rho\right)=0$.

Next, write $\mu^{*}\left(T^{*}\right)=\sum_{j} \zeta_{j} \otimes \psi_{j}$ and

$$
\begin{aligned}
& M^{*}\left(\left(\nu^{\frac{a-1}{2}} \rho\right)^{f_{2}} \times\left(\nu^{\frac{-a+1}{2}} \rho\right)^{k^{*}}\right) \\
& \quad=\sum_{x_{1}, x_{2}} c_{x_{1}, x_{2}}\left(\nu^{\frac{a-1}{2}} \rho\right)^{x_{1}} \times\left(\nu^{\frac{-a+1}{2}} \rho\right)^{x_{2}} \otimes\left(\nu^{\frac{a-1}{2}} \rho\right)^{f_{2}+k^{*}-x_{1}-x_{2}},
\end{aligned}
$$

with $c_{x_{1}, x_{2}}$ the multiplicity (we include the multiplicity in this term only, as it is somewhat awkward to write it otherwise). As

$$
\begin{aligned}
M^{*}( & \left.\delta\left(\left[\nu^{\frac{-a+1}{2}} \rho, \nu^{\frac{a-3}{2}} \rho\right]\right)\right) \\
& =\sum_{r=\frac{-a+1}{2}}^{\frac{a-1}{2}} \sum_{s=r}^{\frac{a-1}{2}} \delta\left(\left[\nu^{r+1} \rho, \nu^{\frac{a-1}{2}} \rho\right]\right) \times \delta\left(\left[\nu^{s}, \nu^{\frac{a-3}{2}} \rho\right]\right) \otimes \delta\left(\left[\nu^{r} \rho, \nu^{s-1} \rho\right]\right),
\end{aligned}
$$

we get

$$
\begin{aligned}
\mu^{*}\left(\mathcal{L}\left(\Delta^{(4)}\right) \times\left(\nu^{\frac{a-1}{2}} \rho\right)^{f_{2}} \times\left(\nu^{\frac{-a+1}{2}} \rho\right)^{k^{*}} \times \delta\left(\left[\nu^{\frac{-a+1}{2}} \rho, \nu^{\frac{a-3}{2}} \rho\right]\right)^{\ell^{*}} \rtimes T^{*}\right) \\
=\sum_{i} \sum_{j} \sum_{x_{1}, x_{2}} \prod_{\ell=1}^{m^{*}} \sum_{r_{\ell}=\frac{-a+1}{2}}^{\frac{a-1}{2}} \sum_{s_{\ell}=r_{\ell}}^{\frac{a-1}{2}} \\
c_{x_{1}, x_{2}} \xi_{i}^{\prime} \times \check{\xi}_{i}^{\prime \prime \prime} \times \zeta_{j} \times\left(\nu^{\frac{a-1}{2}} \rho\right)^{x_{1}} \\
\quad \times\left(\nu^{\frac{-a+1}{2}} \rho\right)^{x_{2}} \times \delta\left(\left[\nu^{r_{\ell}+1} \rho, \nu^{\frac{a-1}{2}} \rho\right]\right) \times \delta\left(\left[\nu^{s_{\ell}}, \nu^{\frac{a-3}{2}} \rho\right]\right) \otimes \ldots
\end{aligned}
$$

Thus, we need

$$
\begin{aligned}
\mathcal{L}\left(\Delta^{(4)}\right) \leq \xi_{i}^{\prime} & \times \check{\xi}_{i}^{\prime \prime \prime} \times \zeta_{j} \\
& \times\left(\nu^{\frac{a-1}{2}} \rho\right)^{x_{1}} \times\left(\nu^{\frac{-a+1}{2}} \rho\right)^{x_{2}} \times \prod_{\ell=1}^{m^{*}} \delta\left(\left[\nu^{r_{\ell}+1} \rho, \nu^{\frac{a-1}{2}} \rho\right]\right) \times \delta\left(\left[\nu^{s_{\ell}}, \nu^{\frac{a-3}{2}} \rho\right]\right) .
\end{aligned}
$$

Now, observe that the supercuspidal support of $\mathcal{L}\left(\Delta_{i, 2}^{(4)}\right)$-which matches the supercuspidal support of $\xi_{i}^{\prime \prime} \otimes \xi_{i}^{\prime \prime \prime}$-must then match that of $\check{\xi}_{i}^{\prime \prime \prime} \times \zeta_{j} \times\left(\nu^{\frac{a-1}{2}} \rho\right)^{x_{1}} \times$ 
$\left(\nu^{\frac{-a+1}{2}} \rho\right)^{x_{2}} \times \prod_{\ell=1}^{m^{*}} \delta\left(\left[\nu^{r_{\ell}+1} \rho, \nu^{\frac{a-1}{2}} \rho\right]\right) \times \delta\left(\left[\nu^{s_{\ell}}, \nu^{\frac{a-3}{2}} \rho\right]\right)$. To simplify notation, write this induced representation as $\left(\nu^{\frac{-a+1}{2}} \rho\right)^{x_{2}} \times \delta\left(\left[\nu^{\frac{-a+1}{2}} \rho, \nu^{\frac{a-3}{2}} \rho\right]\right)^{y} \times \Lambda$, where $y=$ $\mid\left\{\ell \mid s_{\ell}=\frac{-a+1}{2}\right\}$ and $\Lambda$ consists of all the remaining terms. Note that all the terms appearing in $\Lambda$ have nonnegative central exponents. Let us define

$n_{\Lambda}\left(\nu^{x} \rho\right)=$ number of times $\nu^{x} \rho$ appears in the supercuspidal support of $\Lambda$ and

$n_{\mathcal{L}}\left(\nu^{x} \rho\right)=$ number of times $\nu^{x} \rho$ appears in the supercuspidal support of $\mathcal{L}\left(\Delta_{i, 2}^{(4)}\right)$.

We now observe the following:

(1) As the segments comprising $\Delta_{i, 2}^{(4)}$ all have negative central exponent, we have $n_{\mathcal{L}}\left(\nu^{-x} \rho\right) \geq n_{\mathcal{L}}\left(\nu^{x} \rho\right)$ for $x>0$. Further, if $\nu^{-x} \rho$ is a lower segment end, the inequality is strict.

(2) As the segments in $\Lambda$ have nonnegative central exponents, we have $n_{\Lambda}\left(\nu^{-x} \rho\right)$ $\leq n_{\Lambda}\left(\nu^{x} \rho\right)$ for $x>0$.

(3) We have

$$
n_{\mathcal{L}}\left(\nu^{x} \rho\right)=\left\{\begin{array}{l}
n_{\Lambda}\left(\nu^{\frac{-a+1}{2}} \rho\right)+x_{2}+y \text { if } x=\frac{-a+1}{2} \\
n_{\Lambda}\left(\nu^{x} \rho\right)+y \text { if } \frac{-a+3}{2} \leq x \leq \frac{a-3}{2} \\
n_{\Lambda}\left(\nu^{x} \rho\right) \text { otherwise }
\end{array}\right.
$$

Suppose $\nu^{-x} \rho$ is a lower segment end from $\Delta_{i, 2}^{(4)}$. If $-x<\frac{-a+1}{2}$, we have (using (1) and (3) above)

$$
n_{\Lambda}\left(\nu^{-x} \rho\right)=n_{\mathcal{L}}\left(\nu^{-x} \rho\right)>n_{\mathcal{L}}\left(\nu^{x} \rho\right)=n_{\Lambda}\left(\nu^{x} \rho\right)
$$

contradicting (2) above. Similarly, if $\frac{-a+3}{2} \leq-x<0$, we have

$$
n_{\Lambda}\left(\nu^{-x} \rho\right)+y=n_{\mathcal{L}}\left(\nu^{-x} \rho\right)>n_{\mathcal{L}}\left(\nu^{x} \rho\right)=n_{\Lambda}\left(\nu^{x} \rho\right)+y,
$$

again contradicting (2) above. Thus the only possible lower segment ends from $\Delta_{i, 2}^{(4)}$ are $\nu^{\frac{-a+1}{2}} \rho$. However, this would force $g_{\mathcal{L}\left(\Delta_{i, 2}^{(4)}\right)}\left(\nu^{\frac{-a+1}{2}} \rho\right) \neq 0$, another contradiction. Thus we must have $\mathcal{L}\left(\Delta_{i, 2}^{(4)}\right)$ trivial, from which the lemma follows.

Lemma 5.7. Claim 4 holds.

Proof. Write $\mathcal{L}\left(\Delta^{(4)}\right) \times\left(\nu^{\frac{-a+1}{2}} \rho\right)^{k_{\theta_{2}}}=\sum_{i} \mathcal{L}\left(\Delta_{i}\right)$. An argument similar to that used in the proof of Lemma 5.6 gives

$$
\begin{aligned}
\mu_{\mathcal{L}\left(\Delta_{i}\right)}^{*}\left(\mathcal{L}\left(\Delta_{i}\right)\right. & \left.\rtimes L\left(\delta\left(\left[\nu^{\frac{-a+1}{2}} \rho, \nu^{\frac{a-3}{2}} \rho\right]\right)^{\ell_{\theta_{2}}} ; T_{\theta_{2}}\right)\right) \\
& =\mathcal{L}\left(\Delta_{i}\right) \otimes L\left(\delta\left(\left[\nu^{\frac{-a+1}{2}} \rho, \nu^{\frac{a-3}{2}} \rho\right]\right)^{\ell_{\theta_{2}}} ; T_{\theta_{2}}\right) ;
\end{aligned}
$$

the claim may be deduced from this.

\section{Proofs For SECTION 3.4}

In this section, we prove the main results given in section 3.4. In particular, the proofs of Theorem 3.3 and Proposition 3.4 are given in the first and second subsections, respectively.

We begin by recalling some results from [J8].

Proposition 6.1. Consider $\nu^{\frac{a-1}{2}} \rho \rtimes T$, where $a \equiv 2 \operatorname{red}(\rho ; \sigma)+1 \bmod 2$ and $a>2$.

(1) If $m(\rho, a-2)=0$, we have $\nu^{\frac{a-1}{2}} \rho \rtimes T$ irreducible. 
(2) If $m(\rho, a-2)=1$, we have

(a) $\nu^{\frac{a-1}{2}} \rho \rtimes T$ reducible if $m(\rho, a)=0$ or $\varepsilon(\rho, a) \varepsilon(\rho, a-2)^{-1}=1$,

(b) $\nu^{\frac{a-1}{2}} \rho \rtimes T$ irreducible if $\varepsilon(\rho, a) \varepsilon(\rho, a-2)=-1$.

(3) If $m(\rho, a-2) \geq 2$, we have $\nu^{\frac{a-1}{2}} \rho \rtimes T$ reducible.

In the case $a=2$, we have $\nu^{\frac{1}{2}} \rho \rtimes T$ reducible if $m(\rho, 2)=0$ or $\varepsilon(\rho, 2)=1$; if $\varepsilon(\rho, 2)=-1$, we have irreducibility.

Note 6.2. In the case where $a \not \equiv 2 \operatorname{red}(\rho ; \sigma)+1 \bmod 2$, we have the following: $\nu^{\frac{a-1}{2}} \rho \rtimes T$ is reducible if and only if $(\rho, a-2) \in \operatorname{Jord}(T)$. In particular, in the context of (2.1) and (2.3), we have $\nu^{\frac{a-1}{2}} \rho \rtimes \delta$ irreducible, with any reducibility which arises then coming from the reducibility of $\nu^{\frac{a-1}{2}} \rho \times \delta\left(\left[\nu^{\frac{-c_{i}+1}{2}} \rho_{i}, \nu^{\frac{c_{i}-1}{2}} \rho_{i}\right]\right)$ or $\nu^{\frac{a-1}{2}} \rho \times \delta\left(\left[\nu^{\frac{-d_{i}+1}{2}} \rho_{i}^{\prime}, \nu^{\frac{d_{i}-1}{2}} \rho_{i}^{\prime}\right]\right)$ (or with $\nu^{\frac{-a+1}{2}} \check{\rho}$ in place of $\nu^{\frac{a-1}{2}} \rho$ ).

6.1. Proof of Theorem 3.3, In this subsection, we prove Theorem 3.3, which determines $\mu_{\left\{\nu \frac{a-1}{2} \rho\right\}}^{*}\left(L\left(\left(\nu^{\frac{-a+1}{2}} \rho\right)^{k}, \delta\left(\left[\nu^{\frac{-a+1}{2}} \rho, \nu^{\frac{a-3}{2}} \rho\right]\right)^{\ell} ; T\right)\right)$ in the special case when $a=2$. Note that in this case, $\delta\left(\left[\nu^{\frac{-a+1}{2}} \rho, \nu^{\frac{a-3}{2}} \rho\right]\right)=\nu^{\frac{-a+1}{2}} \rho$, so we simply have $\pi=L\left(\left(\nu^{\frac{-a+1}{2}} \rho\right)^{k} ; T\right)$.

Lemma 6.3. Suppose $(\rho, 2) \in \operatorname{Jord}(T)$ with $\varepsilon(\rho, 2)=1$. Then, $\nu^{-\frac{1}{2}} \rho \rtimes L\left(\nu^{-\frac{1}{2}} \rho ; T\right)$ is irreducible.

Proof. Let $\pi=L\left(\nu^{-\frac{1}{2}} \rho ; T\right)$.

We first show that $f_{\pi}\left(\nu^{\frac{1}{2}} \rho\right)=m_{T}(\rho, 2)-1$. To start, we claim that $T \hookrightarrow$ $\nu^{\frac{1}{2}} \rho \rtimes T^{\prime}$, where $T^{\prime}$ has the same data as $T$ except that $m_{T^{\prime}}(\rho, 2)=m_{T}(\rho, 2)-1$ (admissibility for the data for $T^{\prime}$ follows from the appendix to [J8]). To see this, observe that by Lemma 2.2. $T \hookrightarrow\left(\nu^{\frac{1}{2}} \rho\right)^{f_{T}\left(\nu^{\frac{1}{2}} \rho\right)} \rtimes \theta$ implies $T \hookrightarrow \nu^{\frac{1}{2}} \rho \rtimes \lambda$ for some irreducible $\lambda \leq\left(\nu^{\frac{1}{2}} \rho\right)^{f_{T}\left(\nu^{\frac{1}{2}} \rho\right)-1} \rtimes \theta$. From this, we have $f_{T}\left(\nu^{\frac{1}{2}} \rho\right)=1+f_{\lambda}\left(\nu^{\frac{1}{2}} \rho\right)$, implying $\mu_{\left\{\nu^{\frac{1}{2}} \rho\right\}}^{*}(\lambda)=\left(\nu^{\frac{1}{2}} \rho\right)^{f_{T}\left(\nu^{\frac{1}{2}} \rho\right)-1} \otimes \theta$. It now follows from Theorem 3.1 and the uniqueness in (1.2) that $\theta=T^{\prime}$, verifying the claim. Now,

$$
\begin{gathered}
\pi \hookrightarrow \nu^{-\frac{1}{2}} \rho \times \nu^{\frac{1}{2}} \rho \rtimes T^{\prime} \\
\Downarrow(\text { Lemma 2.2) } \\
\pi \hookrightarrow \zeta\left(\left[\nu^{-\frac{1}{2}} \rho, \nu^{\frac{1}{2}} \rho\right]\right) \rtimes T^{\prime} \\
\text { or } \\
\pi \hookrightarrow \delta\left(\left[\nu^{-\frac{1}{2}} \rho, \nu^{\frac{1}{2}} \rho\right]\right) \rtimes T^{\prime} .
\end{gathered}
$$

As the latter would imply $f_{\pi}\left(\nu^{-\frac{1}{2}} \rho\right)=0$, it must be the former, i.e.,

$$
\pi \hookrightarrow \zeta\left(\left[\nu^{-\frac{1}{2}} \rho, \nu^{\frac{1}{2}} \rho\right]\right) \rtimes T^{\prime} .
$$

As $M^{*}\left(\zeta\left(\left[\nu^{-\frac{1}{2}} \rho, \nu^{\frac{1}{2}} \rho\right]\right)\right)$ contains no terms of the form $\nu^{\frac{1}{2}} \rho \otimes \ldots$, it follows immediately that $f_{\pi}\left(\nu^{\frac{1}{2}} \rho\right) \leq f_{T^{\prime}}\left(\nu^{\frac{1}{2}} \rho\right)=m_{T}(\rho, 2)-1$. On the other hand,

$$
\begin{aligned}
\pi \hookrightarrow \zeta\left(\left[\nu^{-\frac{1}{2}} \rho, \nu^{\frac{1}{2}} \rho\right]\right) & \rtimes\left(\left(\nu^{\frac{1}{2}} \rho\right)^{m_{T}(\rho, 2)-1}\right. \\
& \left.\rtimes \theta_{T}\left(\nu^{\frac{1}{2}} \rho\right)\right) \cong\left(\nu^{\frac{1}{2}} \rho\right)^{m_{T}(\rho, 2)-1} \times \zeta\left(\left[\nu^{-\frac{1}{2}} \rho, \nu^{\frac{1}{2}} \rho\right]\right) \rtimes \theta_{T}\left(\nu^{\frac{1}{2}} \rho\right),
\end{aligned}
$$

from which it immediately follows that $f_{\pi}\left(\nu^{\frac{1}{2}} \rho\right) \geq m_{T}(\rho, 2)-1$, implying $f_{\pi}\left(\nu^{\frac{1}{2}} \rho\right)=$ $m_{T}(\rho, 2)-1$, as claimed. 
Next, let $\lambda=L\left(\nu^{-\frac{1}{2}} \rho, \nu^{-\frac{1}{2}} \rho ; T\right)$. As $\zeta\left(\left[\nu^{-\frac{1}{2}} \rho, \nu^{\frac{1}{2}} \rho\right]\right) \rtimes T \hookrightarrow \nu^{-\frac{1}{2}} \rho \times \nu^{\frac{1}{2}} \rho \rtimes T=$ $\nu^{-\frac{1}{2}} \rho \times \nu^{-\frac{1}{2}} \rho \rtimes T$ and

$$
\mu^{*}\left(\zeta\left(\left[\nu^{-\frac{1}{2}} \rho, \nu^{\frac{1}{2}} \rho\right]\right) \rtimes T\right) \geq\left(\nu^{-\frac{1}{2}} \rho \times \nu^{-\frac{1}{2}} \rho\right) \otimes T,
$$

it follows that $\lambda$ is a subquotient of $\zeta\left(\left[\nu^{-\frac{1}{2}} \rho, \nu^{\frac{1}{2}} \rho\right]\right) \rtimes T$; as the induced representation is unitary, $\lambda$ appears as a subrepresentation. As above, it follows from this embedding that $f_{\lambda}\left(\nu^{\frac{1}{2}} \rho\right)=m_{T}(\rho, 2)$.

Now, it follows from the Langlands classification that

$$
\lambda \hookrightarrow \nu^{-\frac{1}{2}} \rho \rtimes L\left(\nu^{-\frac{1}{2}} \rho ; T\right)
$$

as unique irreducible subrepresentation. On the other hand, writing

$$
\mu_{\left\{\nu^{\frac{1}{2}} \rho\right\}}^{*}\left(L\left(\nu^{-\frac{1}{2}} \rho ; T\right)\right)=\left(\nu^{\frac{1}{2}} \rho\right)^{m_{T}(\rho, 2)-1} \otimes \theta,
$$

we then have

$$
\begin{gathered}
\mu_{\left\{\nu^{\frac{1}{2}} \rho\right\}}^{*}(\lambda)=\left(\nu^{\frac{1}{2}} \rho\right)^{m_{T}(\rho, 2)} \otimes \theta \\
\Downarrow \\
\pi \hookrightarrow\left(\nu^{\frac{1}{2}} \rho\right)^{m_{T}(\rho, 2) \rtimes \theta} \\
\Downarrow(\text { Lemma 2.2) } \\
\pi \hookrightarrow \nu^{\frac{1}{2}} \rho \rtimes \Lambda
\end{gathered}
$$

for some irreducible $\Lambda \leq\left(\nu^{\frac{1}{2}} \rho\right)^{m_{T}(\rho, 2)-1} \rtimes \theta$, necessarily having $\mu_{\left\{\nu^{\frac{1}{2}} \rho\right\}}^{*}(\Lambda)=$ $\left(\nu^{\frac{1}{2}} \rho\right)^{m_{T}(\rho, 2)-1} \otimes \theta$. By (1.2), it follows that $\Lambda=L\left(\nu^{-\frac{1}{2}} \rho ; T\right)$. Thus, we have

$$
\lambda \hookrightarrow \nu^{\frac{1}{2}} \rho \rtimes L\left(\nu^{-\frac{1}{2}} \rho ; T\right) .
$$

Now, observe that $L\left(\nu^{-\frac{1}{2}} \rho ; T\right)=L_{\text {quot }}\left(\nu^{\frac{1}{2}} \rho ; T\right)$ (quotient setting of the Langlands classification; see Lemma 1.1 of [J3). As $\lambda=L_{\text {quot }}\left(\nu^{\frac{1}{2}} \rho, \nu^{\frac{1}{2}} \rho ; T\right)$, we see that $\lambda$ is the unique irreducible quotient of $\nu^{\frac{1}{2}} \rho \times \nu^{\frac{1}{2}} \rho \rtimes T$; as $\nu^{\frac{1}{2}} \rho \rtimes L_{\text {quot }}\left(\nu^{\frac{1}{2}} \rho\right.$; $\left.T\right)$ is a quotient of $\nu^{\frac{1}{2}} \rho \times \nu^{\frac{1}{2}} \rho \rtimes T$, it follows that $\lambda$ is the unique irreducible quotient of

$$
\nu^{\frac{1}{2}} \rho \rtimes L_{\text {quot }}\left(\nu^{\frac{1}{2}} \rho ; T\right) \cong \nu^{\frac{1}{2}} \rho \rtimes L\left(\nu^{-\frac{1}{2}} \rho ; T\right) .
$$

As we have just shown that it also appears as a subrepresentation, it then follows from multiplicity one in the Langlands classification that we must have $\nu^{\frac{1}{2}} \rho \rtimes$ $L\left(\nu^{-\frac{1}{2}} \rho ; T\right)$ irreducible, as needed.

Proposition 6.4. Suppose $(\rho, 2) \in \operatorname{Jord}(T)$.

(1) If $\varepsilon(\rho, 2)=1$ (so $\nu^{-\frac{1}{2}} \rho \rtimes T$ reducible), then

$$
\zeta\left(\left[\nu^{-\frac{1}{2}} \rho, \nu^{\frac{1}{2}} \rho\right]\right)^{\ell} \rtimes L\left(\nu^{-\frac{1}{2}} \rho ; T\right)
$$

and

$$
\zeta\left(\left[\nu^{-\frac{1}{2}} \rho, \nu^{\frac{1}{2}} \rho\right]\right)^{\ell} \rtimes L\left(\nu^{-\frac{1}{2}} \rho, \nu^{-\frac{1}{2}} \rho ; T\right)
$$

are irreducible.

(2) If $\varepsilon(\rho, 2)=-1$ (so $\nu^{-\frac{1}{2}} \rho \rtimes T$ irreducible, $)$, then

$$
\zeta\left(\left[\nu^{-\frac{1}{2}} \rho, \nu^{\frac{1}{2}} \rho\right]\right)^{\ell} \rtimes T
$$

and

$$
\zeta\left(\left[\nu^{-\frac{1}{2}} \rho, \nu^{\frac{1}{2}} \rho\right]\right)^{\ell} \rtimes L\left(\nu^{-\frac{1}{2}} \rho ; T\right)
$$

are irreducible. 
Proof. We start with $\zeta\left(\left[\nu^{-\frac{1}{2}} \rho, \nu^{\frac{1}{2}} \rho\right]\right)^{\ell} \rtimes L\left(\nu^{-\frac{1}{2}} \rho ; T\right)$ when $\varepsilon(\rho, 2)=1$. Observe that using Lemma 6.3,

$$
\begin{aligned}
\zeta\left(\left[\nu^{-\frac{1}{2}} \rho, \nu^{\frac{1}{2}} \rho\right]\right)^{\ell} \rtimes L\left(\nu^{-\frac{1}{2}} \rho ; T\right) & \hookrightarrow \zeta\left(\left[\nu^{-\frac{1}{2}} \rho, \nu^{\frac{1}{2}} \rho\right]\right)^{\ell-1} \times \nu^{-\frac{1}{2}} \rho \times \nu^{\frac{1}{2}} \rho \rtimes L\left(\nu^{-\frac{1}{2}} \rho ; T\right) \\
& \cong \zeta\left(\left[\nu^{-\frac{1}{2}} \rho, \nu^{\frac{1}{2}} \rho\right]\right)^{\ell-1} \times \nu^{-\frac{1}{2}} \rho \times \nu^{-\frac{1}{2}} \rho \rtimes L\left(\nu^{-\frac{1}{2}} \rho ; T\right) \\
& \cong \nu^{-\frac{1}{2}} \rho \times \nu^{-\frac{1}{2}} \rho \times \zeta\left(\left[\nu^{-\frac{1}{2}} \rho, \nu^{\frac{1}{2}} \rho\right]\right)^{\ell-1} \rtimes L\left(\nu^{-\frac{1}{2}} \rho ; T\right) \\
& \vdots(\text { iterating }) \\
& \hookrightarrow\left(\nu^{-\frac{1}{2}} \rho\right)^{2 \ell} \rtimes L\left(\nu^{-\frac{1}{2}} \rho ; T\right),
\end{aligned}
$$

which has unique irreducible subrepresentation $L\left(\left(\nu^{-\frac{1}{2}} \rho\right)^{2 \ell+1} ; T\right)$ by the Langlands classification. Therefore, $L\left(\left(\nu^{-\frac{1}{2}} \rho\right)^{2 \ell+1} ; T\right)$ appears in $\zeta\left(\left[\nu^{-\frac{1}{2}} \rho, \nu^{\frac{1}{2}} \rho\right]\right)^{\ell} \rtimes L\left(\nu^{-\frac{1}{2}} \rho ; T\right)$ as unique irreducible subrepresentation. Further, it follows from multiplicity one for the Langlands classification that it appears with multiplicity one in $\zeta\left(\left[\nu^{-\frac{1}{2}} \rho, \nu^{\frac{1}{2}} \rho\right]\right)^{\ell}$ $\rtimes L\left(\nu^{-\frac{1}{2}} \rho ; T\right)$ as well.

Now, applying the same argument but starting with $\check{T}$, we have

$$
L\left(\left(\nu^{-\frac{1}{2}} \rho\right)^{2 \ell+1} ; \check{T}\right) \hookrightarrow \zeta\left(\left[\nu^{-\frac{1}{2}} \rho, \nu^{\frac{1}{2}} \rho\right]\right)^{\ell} \rtimes L\left(\nu^{-\frac{1}{2}} \rho ; \check{T}\right)
$$

as unique irreducible subrepresentation. Taking contragredients or their complex conjugates,

$$
L\left(\left(\nu^{-\frac{1}{2}} \rho\right)^{2 \ell+1} ; \check{T}\right)^{\complement} \cong L\left(\left(\nu^{-\frac{1}{2}} \rho\right)^{2 \ell+1} ; T\right)
$$

(Lemma 1.1 of [J3]) appears as the unique irreducible quotient of

$$
\left(\zeta\left(\left[\nu^{-\frac{1}{2}} \rho, \nu^{\frac{1}{2}} \rho\right]\right)^{\ell} \rtimes L\left(\nu^{-\frac{1}{2}} \rho ; \check{T}\right)\right)^{\frown} \cong \zeta\left(\left[\nu^{-\frac{1}{2}} \rho, \nu^{\frac{1}{2}} \rho\right]\right)^{\ell} \rtimes L\left(\nu^{-\frac{1}{2}} \rho ; T\right) .
$$

This contradicts multiplicity one unless

$$
\zeta\left(\left[\nu^{-\frac{1}{2}} \rho, \nu^{\frac{1}{2}} \rho\right]\right)^{\ell} \rtimes L\left(\nu^{-\frac{1}{2}} \rho ; T\right)=L\left(\left(\nu^{-\frac{1}{2}} \rho\right)^{2 \ell+1} ; T\right)
$$

(irreducible), as needed.

The case $\zeta\left(\left[\nu^{-\frac{1}{2}} \rho, \nu^{\frac{1}{2}} \rho\right]\right)^{\ell} \rtimes T$ when $\varepsilon(\rho, 2)=-1$ may be done similarly.

For the remaining cases $-\zeta\left(\left[\nu^{-\frac{1}{2}} \rho, \nu^{\frac{1}{2}} \rho\right]\right)^{\ell} \rtimes L\left(\nu^{-\frac{1}{2}} \rho, \nu^{-\frac{1}{2}} \rho ; T\right)$ when $\varepsilon(\rho, 2)=1$ and $\zeta\left(\left[\nu^{-\frac{1}{2}} \rho, \nu^{\frac{1}{2}} \rho\right]\right)^{\ell} \rtimes L\left(\nu^{-\frac{1}{2}} \rho ; T\right)$ when $\varepsilon(\rho, 2)=-1$-note that by Lemma 6.3 and Proposition 6.1. we have $L\left(\nu^{-\frac{1}{2}} \rho, \nu^{-\frac{1}{2}} \rho ; T\right)=\nu^{-\frac{1}{2}} \rho \rtimes L\left(\nu^{-\frac{1}{2}} \rho ; T\right)$ (irreducible) for $\varepsilon(\rho, 2)=1$ and $L\left(\nu^{-\frac{1}{2}} \rho ; T\right)=\nu^{-\frac{1}{2}} \rho \rtimes T$ (irreducible) when $\varepsilon(\rho, 2)=-1$. Using these observations, these cases may also be done using an argument like that above.

Lemma 6.5. Suppose $(\rho, 2) \in \operatorname{Jord}(T)$ with $\varepsilon(\rho, 2)=1$. Let $\pi=L\left(\nu^{-\frac{1}{2}} \rho ; T\right)$ and $m=m(\rho, 2)$. Then,

$$
\mu_{\left\{\nu^{\frac{1}{2}} \rho\right\}}^{*}(\pi)=\left\{\begin{array}{l}
\left(\nu^{\frac{1}{2}} \rho\right)^{m-1} \otimes L\left(\nu^{-\frac{1}{2}} \rho ; T^{\prime}\right) \text { if } m \text { odd } \\
\left(\nu^{\frac{1}{2}} \rho\right)^{m-1} \otimes L\left(\nu^{-\frac{1}{2}} \rho, \nu^{-\frac{1}{2}} \rho ; T^{\prime \prime}\right) \text { if } m \text { even },
\end{array}\right.
$$

where $m_{T^{\prime}}(\rho, 2)=1, m_{T^{\prime \prime}}(\rho, 2)=0$, the remaining multiplicities for $T^{\prime}, T^{\prime \prime}$ match those for $T, \varepsilon_{T^{\prime}}=\varepsilon_{T}$, and $\varepsilon_{T^{\prime \prime}}$ is the restriction of $\varepsilon_{T}$.

Proof. Recall from the proof of Lemma 6.3 that $f_{\pi}\left(\nu^{\frac{1}{2}} \rho\right)=m-1$.

We first address the case $m$ odd. In this case, we have $T \cong \delta\left(\left[\nu^{-\frac{1}{2}} \rho, \nu^{\frac{1}{2}} \rho\right]\right)^{\frac{m-1}{2}} \rtimes$ $T^{\prime}$. Therefore, $\pi \leq \delta\left(\left[\nu^{-\frac{1}{2}} \rho, \nu^{\frac{1}{2}} \rho\right]\right)^{\frac{m-1}{2}} \rtimes L\left(\nu^{-\frac{1}{2}} \rho ; T^{\prime}\right)$. As

$$
\mu_{\left\{\nu^{\frac{1}{2}} \rho\right\}}^{*}\left(\delta\left(\left[\nu^{-\frac{1}{2}} \rho, \nu^{\frac{1}{2}} \rho\right]\right)^{\frac{m-1}{2}} \rtimes L\left(\nu^{-\frac{1}{2}} \rho ; T^{\prime}\right)\right)=\left(\nu^{\frac{1}{2}} \rho\right)^{m-1} \otimes L\left(\nu^{-\frac{1}{2}} \rho ; T^{\prime}\right)
$$


and $f_{\pi}\left(\nu^{\frac{1}{2}} \rho\right)=m-1$, it then follows that

$$
\mu_{\left\{\nu \frac{1}{2} \rho\right\}}^{*}(\pi)=\left(\nu^{\frac{1}{2}} \rho\right)^{m-1} \otimes L\left(\nu^{-\frac{m-1}{2}} \rho ; T^{\prime}\right),
$$

as needed.

Now, suppose $m$ is even. First, if $m=2$, we have

$$
\begin{aligned}
\pi & \hookrightarrow \nu^{-\frac{1}{2}} \rho \rtimes T \\
& \hookrightarrow \nu^{-\frac{1}{2}} \rho \times \delta\left(\left[\nu^{-\frac{1}{2}} \rho, \nu^{\frac{1}{2}} \rho\right]\right) \rtimes T^{\prime \prime} \\
& \cong \delta\left(\left[\nu^{-\frac{1}{2}} \rho, \nu^{\frac{1}{2}} \rho\right]\right) \times \nu^{-\frac{1}{2}} \rho \rtimes T^{\prime \prime} \\
& \hookrightarrow \nu^{\frac{1}{2}} \rho \times \nu^{-\frac{1}{2}} \rho \times \nu^{-\frac{1}{2}} \rho \rtimes T^{\prime \prime} .
\end{aligned}
$$

As $f_{\pi}\left(\nu^{\frac{1}{2}} \rho\right)=1$, it follows from Lemma 2.4 that

$$
\mu_{\left\{\nu^{\frac{1}{2}} \rho\right\}}^{*}(\pi)=\nu^{\frac{1}{2}} \rho \otimes L\left(\nu^{-\frac{1}{2}} \rho, \nu^{-\frac{1}{2}} \rho ; T^{\prime \prime}\right),
$$

as needed.

If $m>2$ even, we can now argue as in the case $m$ odd. We have $T \cong$ $\delta\left(\left[\nu^{-\frac{1}{2}} \rho, \nu^{\frac{1}{2}} \rho\right]\right)^{\frac{m-2}{2}} \rtimes T_{2}$, where $m_{T_{2}}(\rho, 2)=2$ and the remaining data matches that for $T$. Then, $\pi \leq \delta\left(\left[\nu^{-\frac{1}{2}} \rho, \nu^{\frac{1}{2}} \rho\right]\right)^{\frac{m-2}{2}} \rtimes L\left(\nu^{-\frac{1}{2}} \rho ; T_{2}\right)$. From above,

$$
\mu_{\left\{\nu^{\frac{1}{2}}\right\}}^{*}\left(L\left(\nu^{-\frac{1}{2}} \rho ; T_{2}\right)\right)=\nu^{\frac{1}{2}} \rho \otimes L\left(\nu^{-\frac{1}{2}} \rho, \nu^{-\frac{1}{2}} \rho ; T^{\prime \prime}\right) .
$$

One then has

$$
\begin{gathered}
\mu_{\left\{\nu^{\frac{1}{2}} \rho\right\}}^{*}\left(\delta\left(\left[\nu^{-\frac{1}{2}} \rho, \nu^{\frac{1}{2}} \rho\right]\right)^{\frac{m-2}{2}} \rtimes L\left(\nu^{-\frac{1}{2}} \rho ; T_{2}\right)\right)=\left(\nu^{\frac{1}{2}} \rho\right)^{m-1} \otimes L\left(\nu^{-\frac{1}{2}} \rho, \nu^{-\frac{1}{2}} \rho ; T^{\prime \prime}\right) \\
\Downarrow \\
\mu_{\left\{\nu^{\frac{1}{2}} \rho\right\}}^{*}(\pi)=\left(\nu^{\frac{1}{2}} \rho\right)^{m-1} \otimes L\left(\nu^{-\frac{1}{2}} \rho, \nu^{-\frac{1}{2}} \rho ; T^{\prime \prime}\right),
\end{gathered}
$$

(again noting $\left.f_{\pi}\left(\nu^{\frac{1}{2}} \rho\right)=m-1\right)$, as needed.

Proof of Theorem 3.3. For (1), we first claim that $f_{L\left(\nu^{-\frac{1}{2}} \rho ; T\right)}\left(\nu^{\frac{1}{2}} \rho\right)=0$. Suppose this were not the case. As $f_{T}\left(\nu^{\frac{1}{2}} \rho\right)=0$, it then follows from $L\left(\nu^{-\frac{1}{2}} \rho ; T\right) \leq \nu^{\frac{1}{2}} \rho \rtimes T$ that

$$
\mu_{\left\{\nu^{\frac{1}{2}} \rho\right\}}^{*}\left(L\left(\nu^{-\frac{1}{2}} \rho ; T\right)\right)=\nu^{\frac{1}{2}} \rho \otimes T .
$$

Therefore, by (1.2)

$$
L\left(\nu^{-\frac{1}{2}} \rho ; T\right) \hookrightarrow \nu^{\frac{1}{2}} \rho \rtimes T .
$$

As $L\left(\nu^{-\frac{1}{2}} \rho ; T\right)=L_{\text {quot }}\left(\nu^{\frac{1}{2}} \rho ; T\right)$, we have $L\left(\nu^{-\frac{1}{2}} \rho ; T\right)$ appearing as both the unique irreducible quotient and as a subrepresentation in $\nu^{\frac{1}{2}} \rho \rtimes T$. This contradicts multiplicity one in the Langlands classification unless $\nu^{\frac{1}{2}} \rho \rtimes T$ is irreducible. However, by Proposition 6.1, that is not the case. Thus, we must have $f_{L\left(\nu^{-\frac{1}{2}} \rho ; T\right)}\left(\nu^{\frac{1}{2}} \rho\right)=0$, as claimed.

For $(2)-(5)$ when $\varepsilon(\rho, 2)=-1$, observe that from Proposition 6.4

$$
\pi=\zeta\left(\left[\nu^{-\frac{1}{2}} \rho, \nu^{\frac{1}{2}} \rho\right]\right)^{\frac{k}{2}} \rtimes T
$$

when $k$ is even and

$$
\pi=\zeta\left(\left[\nu^{-\frac{1}{2}} \rho, \nu^{\frac{1}{2}} \rho\right]\right)^{\frac{k-1}{2}} \times \nu^{\frac{1}{2}} \rho \rtimes T
$$


when $k$ is odd. Writing $\mu_{\left\{\nu^{\frac{1}{2}} \rho\right\}}^{*}(T)=\left(\nu^{\frac{1}{2}} \rho\right)^{m-1} \otimes \theta_{T}\left(\nu^{\frac{1}{2}} \rho\right)$, we see that

$$
\mu_{\left\{\nu^{\frac{1}{2}} \rho\right\}}^{*}(\pi)=\left\{\begin{array}{l}
\left(\nu^{\frac{1}{2}} \rho\right)^{m-1} \otimes \zeta\left(\left[\nu^{-\frac{1}{2}} \rho, \nu^{\frac{1}{2}} \rho\right]\right)^{\frac{k}{2}} \rtimes \theta_{T}\left(\nu^{\frac{1}{2}} \rho\right) \text { if } k \text { even, } \\
\left(\nu^{\frac{1}{2}} \rho\right)^{m} \otimes \zeta\left(\left[\nu^{-\frac{1}{2}} \rho, \nu^{\frac{1}{2}} \rho\right]\right)^{\frac{k-1}{2}} \rtimes \theta_{T}\left(\nu^{\frac{1}{2}} \rho\right) \text { if } k \text { odd, }
\end{array}\right.
$$

noting that it follows from (1.2) that the induced representations on the right-hand side above are necessarily irreducible. By Theorem 3.1 ,

$$
\theta_{T}\left(\nu^{\frac{1}{2}} \rho\right)=\left\{\begin{array}{l}
T^{\prime} \text { if } m \text { odd, } \\
L\left(\nu^{-\frac{1}{2}} \rho ; T^{\prime \prime}\right) \text { if } m \text { even, }
\end{array}\right.
$$

from which the result then follows.

The argument when $\varepsilon(\rho, 2)=1$ is similar, but uses

$$
\pi=\left\{\begin{array}{l}
\zeta\left(\left[\nu^{-\frac{1}{2}} \rho, \nu^{\frac{1}{2}} \rho\right]\right)^{\frac{k-1}{2}} \rtimes L\left(\nu^{-\frac{1}{2}} \rho ; T\right) \text { if } k \text { odd }, \\
\zeta\left(\left[\nu^{-\frac{1}{2}} \rho, \nu^{\frac{1}{2}} \rho\right]\right)^{\frac{k-2}{2}} \times \nu^{\frac{1}{2}} \rho \rtimes L\left(\nu^{-\frac{1}{2}} \rho ; T\right) \text { if } k \text { even, }
\end{array}\right.
$$

(by Lemma 6.3 and Proposition 6.4) and Lemma 6.5.

6.2. Proof of Proposition 3.4. We use notation as in Proposition 3.4

Proof of Proposition 3.4. First, observe that (noting $a>3 \Rightarrow \nu^{\frac{-a+1}{2}} \rho \times \nu^{\frac{a-3}{2}} \rho$ irreducible)

$$
\begin{aligned}
& \pi \hookrightarrow\left(\nu^{\frac{-a+1}{2}} \rho\right)^{k} \times \delta\left(\left[\nu^{\frac{-a+1}{2}} \rho, \nu^{\frac{a-3}{2}} \rho\right]\right)^{\ell} \rtimes T
\end{aligned}
$$

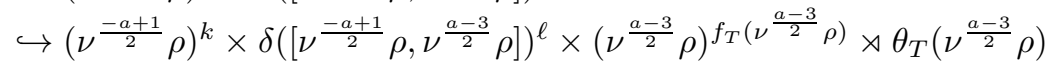

$$
\begin{aligned}
& \cong\left(\nu^{\frac{a-3}{2}} \rho\right)^{f_{T}\left(\nu^{\frac{a-3}{2}} \rho\right)} \times \delta\left(\left[\nu^{\frac{-a+1}{2}} \rho, \nu^{\frac{a-3}{2}} \rho\right]\right)^{\ell} \times\left(\nu^{\frac{-a+1}{2}} \rho\right)^{k} \rtimes \theta_{T}\left(\nu^{\frac{a-3}{2}} \rho\right) \\
& \Downarrow \\
& f_{\pi}\left(\nu^{\frac{a-3}{2}} \rho\right)=\ell+f_{T}\left(\nu^{\frac{a-3}{2}} \rho\right) \text {. }
\end{aligned}
$$

Therefore, we have $f_{\pi}\left(\nu^{\frac{a-3}{2}} \rho\right)=0$ if and only if $\ell=0$ and $f_{T}\left(\nu^{\frac{a-3}{2}} \rho\right)=0$. By Theorem 3.1, this means either (i) $(\rho, a-2) \notin \operatorname{Jord}(T)$ or (ii) $(\rho, a-2),(\rho, a-4) \in$ $\operatorname{Jord}(T)$ with $m_{T}(\rho, a-2)=1$ and $\varepsilon_{T}(\rho, a-2) \varepsilon_{T}(\rho, a-4)^{-1}=-1$ (interpreted as $\varepsilon_{T}(\rho, 2)=-1$ for $\left.a=4\right)$. This verifies the conditions for $f_{\pi}\left(\nu^{\frac{a-3}{2}} \rho\right)=0$ in the statement of Proposition 3.4. We next turn to the calculation of $\mu_{\left\{\nu \frac{a-1}{2} \rho\right\}}^{*}(\pi)$ when these conditions are met.

If $(\rho, a-2) \notin \operatorname{Jord}(T)$, we have $\nu^{\frac{-a+1}{2}} \rho \rtimes T$ irreducible (Proposition 6.1) and $\mu_{\left\{\nu^{\frac{a-1}{2}} \rho\right\}}^{*}(T)=\left(\nu^{\frac{a-1}{2}} \rho\right)^{m_{T}(\rho, a)} \otimes T_{1}$ (Theorem 3.1). As $a>3$, we have $\nu^{\frac{-a+1}{2} \rho \times}$ $\nu^{\frac{a-1}{2}} \rho$ irreducible. Thus,

$$
\pi \hookrightarrow\left(\nu^{\frac{-a+1}{2}} \rho\right)^{k} \times T \cong\left(\nu^{\frac{a-1}{2}} \rho\right)^{k} \rtimes T \hookrightarrow\left(\nu^{\frac{a-1}{2}} \rho\right)^{k+m_{T}(\rho, a)} \rtimes T_{1},
$$

from which the results for (1) and (5) follow.

For (2) and (3), we have $(\rho, a-2) \in \operatorname{Jord}(T)$ and $\varepsilon(\rho, a) \varepsilon_{T}(\rho, a-2)^{-1}=-1$. In this case, we again have $\nu^{\frac{a-1}{2}} \rho \rtimes T$ irreducible, and the argument proceeds as for $(1),(5)$ above (the case $(\rho, a-2) \notin \operatorname{Jord}(T))$.

For (4), we have $(\rho, a-2) \in \operatorname{Jord}(T)$ and $\varepsilon(\rho, a) \varepsilon_{T}(\rho, a-2)^{-1}=1$. Note that we also have $m_{T}(\rho, a-2)=1$ and $\varepsilon(\rho, a-2) \varepsilon_{T}(\rho, a-4)^{-1}=-1$ (suitably interpreted for $a=4)$. By Corollary 3.4.4 of [J7],

$$
T \hookrightarrow \delta\left(\left[\nu^{\frac{-a+3}{2}} \rho, \nu^{\frac{a-1}{2}} \rho\right]\right) \rtimes T^{\prime}
$$


( $T^{\prime}$ as described there). Therefore,

$$
\begin{gathered}
\pi \hookrightarrow\left(\nu^{\frac{-a+1}{2}} \rho\right)^{k} \times \delta\left(\left[\nu \frac{-a+3}{2} \rho, \nu^{\frac{a-1}{2}} \rho\right]\right) \rtimes T^{\prime} \\
\Downarrow(\operatorname{Lemma} 2.2) \\
\pi \hookrightarrow\left(\nu^{\frac{-a+1}{2}} \rho\right)^{k-1} \times \delta\left(\left[\nu^{\frac{-a+1}{2}} \rho, \nu^{\frac{a-1}{2}} \rho\right]\right) \rtimes T^{\prime} \\
\text { or } \\
\pi \hookrightarrow\left(\nu^{\frac{-a+1}{2}} \rho\right)^{k-1} \times \mathcal{L}\left(\nu^{\frac{-a+1}{2}} \rho, \delta\left(\left[\nu^{\frac{-a+3}{2}} \rho, \nu^{\frac{a-1}{2}} \rho\right]\right)\right) \rtimes T^{\prime} .
\end{gathered}
$$

As the former would imply $\pi=L\left(\left(\nu^{\frac{-a+1}{2}} \rho\right)^{k-1} ; \mathcal{T}\right)$ for some irreducible $\mathcal{T} \leq$ $\delta\left(\left[\nu^{\frac{-a+1}{2}} \rho, \nu^{\frac{a-1}{2}} \rho\right]\right) \rtimes T^{\prime}$ by the Langlands classification, it must be the latter. As $f_{T^{\prime}}\left(\nu^{\frac{a-1}{2}} \rho\right)=m_{T^{\prime}}(\rho, a)=m_{T}(\rho, a)-1$, we have

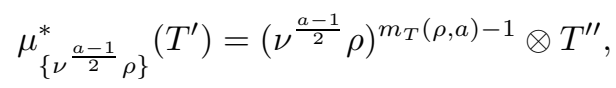

where $m_{T^{\prime \prime}}(\rho, a-2)=m_{T^{\prime}}(\rho, a)=m_{T}(\rho, a)-1, m_{T^{\prime \prime}}(\rho, a)=0$, remaining multiplicities matching $T$, and $\varepsilon_{T^{\prime \prime}}$ the restriction of $\varepsilon_{T}$. Noting the irreducibility of $\nu^{\frac{-a+1}{2}} \rho \rtimes T^{\prime}$ (Proposition 6.1) and $\mathcal{L}\left(\nu^{\frac{-a+1}{2}} \rho, \delta\left(\left[\nu^{\frac{-a+3}{2}} \rho, \nu^{\frac{a-1}{2}} \rho\right]\right)\right) \times \nu^{ \pm\left(\frac{a-1}{2}\right)} \rho$ (Lemma 1.3.3 of [J5] and Lemma 3.6 of [J8]), we then have

$$
\begin{aligned}
\pi & \hookrightarrow \mathcal{L}\left(\nu^{\frac{-a+1}{2}} \rho, \delta\left(\left[\nu^{\frac{-a+3}{2}} \rho, \nu^{\frac{a-1}{2}} \rho\right]\right)\right) \times\left(\nu^{\frac{-a+1}{2}} \rho\right)^{k-1} \rtimes T^{\prime} \\
& \cong \mathcal{L}\left(\nu^{\frac{-a+1}{2}} \rho, \delta\left(\left[\nu^{\frac{-a+3}{2}} \rho, \nu^{\frac{a-1}{2}} \rho\right]\right)\right) \times\left(\nu^{\frac{a-1}{2}} \rho\right)^{k-1} \rtimes T^{\prime} \\
& \hookrightarrow \mathcal{L}\left(\nu^{\frac{-a+1}{2}} \rho, \delta\left(\left[\nu^{\frac{-a+3}{2}} \rho, \nu^{\frac{a-1}{2}} \rho\right]\right)\right) \times\left(\nu^{\frac{a-1}{2}} \rho\right)^{k+m_{T}(\rho, a)-2} \rtimes T^{\prime \prime} \\
& \cong\left(\nu^{\frac{a-1}{2}} \rho\right)^{k+m_{T}(\rho, a)-2} \times \mathcal{L}\left(\nu^{\frac{-a+1}{2}} \rho, \delta\left(\left[\nu^{\frac{-a+3}{2}} \rho, \nu^{\frac{a-1}{2}} \rho\right]\right)\right) \rtimes T^{\prime \prime} \\
& \hookrightarrow\left(\nu^{\frac{a-1}{2}} \rho\right)^{k+m_{T}}(\rho, a)-1 \times \mathcal{L}\left(\nu^{\frac{-a+1}{2}} \rho, \delta\left(\left[\nu^{\frac{-a+3}{2}} \rho, \nu^{\frac{a-3}{2}} \rho\right]\right)\right) \rtimes T^{\prime \prime} .
\end{aligned}
$$

Note that as neither $M^{*}\left(\mathcal{L}\left(\nu^{\frac{-a+1}{2}} \rho, \delta\left(\left[\nu^{\frac{-a+3}{2}} \rho, \nu^{\frac{a-3}{2}} \rho\right]\right)\right)\right)$ nor $\mu^{*}\left(T^{\prime \prime}\right)$ contains terms of the form $\nu^{\frac{a-1}{2}} \rho \otimes \ldots$, it follows that $f_{\pi}\left(\nu^{\frac{a-1}{2}} \rho\right)=k+m_{T}(\rho, a)-1$.

Observe that if $m_{T^{\prime \prime}}(\rho, a-2)=m_{T}(\rho, a)-1>0$, then $\delta\left(\left[\nu^{\frac{-a+3}{2}} \rho, \nu^{\frac{a-3}{2}} \rho\right]\right) \rtimes T^{\prime \prime}$ is irreducible and

$$
\begin{gathered}
\pi \hookrightarrow\left(\nu^{\frac{a-1}{2}} \rho\right)^{k+m_{T}(\rho, a)-1} \times \nu^{\frac{-a+1}{2}} \rho \times \delta\left(\left[\nu^{\frac{-a+3}{2}} \rho, \nu^{\frac{a-3}{2}} \rho\right]\right) \rtimes T^{\prime \prime} \\
\Downarrow(\operatorname{Lemma} 2.4) \\
\mu_{\left\{\nu^{\frac{a-1}{2}} \rho\right\}}^{*}(\pi)=\left(\nu^{\frac{a-1}{2}} \rho\right)^{k+m_{T}(\rho, a)-1} \otimes L\left(\nu^{\frac{-a+1}{2}} \rho ; \delta\left(\left[\nu^{\frac{-a+3}{2}} \rho, \nu^{\frac{a-3}{2}} \rho\right]\right) \rtimes T^{\prime \prime}\right) .
\end{gathered}
$$

The data for $\delta\left(\left[\nu^{\frac{-a+3}{2}} \rho, \nu^{\frac{a-3}{2}} \rho\right]\right) \rtimes T^{\prime \prime}$ matches that for $T_{4}$, finishing the proof in the case $m_{T}(\rho, a)>1$.

If $m_{T}(\rho, a)=1$, then $\delta\left(\left[\nu^{\frac{-a+3}{2}} \rho, \nu^{\frac{a-3}{2}} \rho\right]\right) \rtimes T^{\prime \prime}$ is reducible. The same argument as above shows that

$$
\mu_{\left\{\nu \frac{a-1}{2} \rho\right\}}^{*}(\pi)=\left(\nu^{\frac{a-1}{2}} \rho\right)^{k+m_{T}(\rho, a)-1} \otimes L\left(\nu^{\frac{-a+1}{2}} \rho ; T^{*}\right)
$$

for some $T^{*} \leq \delta\left(\left[\nu^{\frac{-a+3}{2}} \rho, \nu^{\frac{a-3}{2}} \rho\right]\right) \rtimes T^{\prime \prime}$. To show $T^{*}=T_{4}$, we must show that $\varepsilon_{T^{*}}(\rho, a-2) \varepsilon_{T^{*}}(\rho, a-4)=-1$. To this end, observe that if $\theta_{\pi}\left(\nu^{\frac{a-1}{2}} \rho\right)=L\left(\nu^{\frac{-a+1}{2}} \rho ; T^{*}\right)$ with $\varepsilon_{T^{*}}(\rho, a-2) \varepsilon_{T^{*}}(\rho, a-4)=1$, then

$$
\mu_{\left\{\nu \frac{a-3}{2} \rho\right\}}^{*}\left(L\left(\nu^{\frac{-a+1}{2}} \rho ; T^{*}\right)\right)=\left(\nu^{\frac{a-3}{2}} \rho\right)^{2} \otimes L\left(\nu^{\frac{-a+1}{2}} \rho ; T^{* *}\right),
$$

where $m_{T^{* *}}(\rho, a-2)=0, m_{T^{* *}}(\rho, a-4)=m_{T^{*}}(\rho, a-4)+2$, and remaining data matching that of $T^{*}$. Irreducibility (Proposition 6.1) then tells us $\nu^{\frac{-a+1}{2}} \rho \rtimes T^{* *}=$ $L\left(\nu^{\frac{-a+1}{2}} \rho ; T^{*}\right)$. For $X=\left\{\nu^{\frac{a-3}{2}} \rho, \nu^{\frac{a-1}{2}} \rho\right\}$, we then have

$$
f_{\pi}(X) \geq\left(k+m_{T}(\rho, a)-1\right)+2+1=k+3 .
$$


However, as $\pi \leq\left(\nu^{\frac{-a+1}{2}} \rho\right)^{k} \rtimes T$, we have

$$
f_{\pi}(X) \leq k+f_{T}(X)
$$

as $\varepsilon_{T}(\rho, a-2) \varepsilon_{T}(\rho, a-4)^{-1}=-1$ (and noting $m_{T}(\rho, a-2)=m_{T}(\rho, a)=1$ ), we see that $f_{T}(X)=2$. Therefore,

$$
f_{\pi}(X) \leq k+2
$$

a contradiction. Thus we cannot have $\varepsilon_{T^{*}}(\rho, a-2) \varepsilon_{T^{*}}(\rho, a-4)=1$. This gives $\varepsilon_{T^{*}}(\rho, a-2) \varepsilon_{T^{*}}(\rho, a-4)^{-1}=-1$, as needed, finishing this case.

The claim for (6) follows directly from the following observations: (a) $f_{L\left(\nu \frac{-a+1}{2} \rho ; T\right)}\left(\nu^{\frac{a-1}{2}} \rho\right)=0$, and (b) $\nu^{\frac{-a+1}{2}} \rho \rtimes L\left(\nu^{\frac{-a+1}{2}} \rho ; T\right)$ is irreducible, which we establish below.

To see that $f_{L\left(\nu \frac{-a+1}{2} \rho ; T\right)}\left(\nu^{\frac{a-1}{2}} \rho\right)=0$, we argue indirectly. Suppose not-then $\mu_{\left\{\nu \frac{a-1}{2} \rho\right\}}^{*}\left(L\left(\nu^{\frac{-a+1}{2}} \rho ; T\right)\right)=\nu^{\frac{a-1}{2}} \rho \otimes T$. It then follows that $L\left(\nu^{\frac{-a+1}{2}} \rho ; T\right) \hookrightarrow \nu^{\frac{a-1}{2}} \rho \rtimes$

$T$. However, $L\left(\nu^{\frac{-a+1}{2}} \rho ; T\right)$ also appears as the unique irreducible quotient (Langlands quotient) in $\nu^{\frac{a-1}{2}} \rho \rtimes T$. Since it appears with multiplicity one (by the Langlands classification), we must have $\nu^{\frac{a-1}{2}} \rho \rtimes T$ irreducible. However, by Proposition 6.1 that is not the case. Thus $f_{L\left(\nu \frac{-a+1}{2} \rho ; T\right)}\left(\nu^{\frac{a-1}{2}} \rho\right)=0$, as claimed.

To see that $\nu^{\frac{-a+1}{2}} \rho \rtimes L\left(\nu^{\frac{-a+1}{2}} \rho ; T\right)$ is irreducible, first observe that by Proposition 5.1, an irreducible subquotient of $\nu^{\frac{-a+1}{2}} \rho \rtimes L\left(\nu^{\frac{-a+1}{2}} \rho ; T\right)$ must have one of the following forms: $\pi_{1}=L\left(\left(\nu^{\frac{-a+1}{2}} \rho\right)^{2} ; T\right), \pi_{2}=L\left(\nu^{\frac{-a+1}{2}} \rho, \delta\left(\left[\nu^{\frac{-a+1}{2}} \rho, \nu^{\frac{a-3}{2}} \rho\right]\right) ; T_{1}\right)$, $\pi_{3}=L\left(\delta\left(\left[\nu^{\frac{-a+1}{2}} \rho, \nu^{\frac{a-3}{2}} \rho\right]\right)^{2} ; T_{2}\right), \pi_{4}=L\left(\nu^{\frac{-a+1}{2}} \rho ; T_{3}\right), \pi_{5}=L\left(\delta\left(\left[\nu^{\frac{-a+1}{2}} \rho, \nu^{\frac{a-3}{2}} \rho\right]\right) ; T_{4}\right)$, or $\pi_{6}=T_{5}$. Of course, $\pi_{1}$ appears with multiplicity one by the Langlands classification. Thus it remains to show that none of the other possibilities acutally occur.

To show the other possibilities cannot occur, we use Proposition 2.4.1 of [J7], which tells how $\operatorname{Jord}(T)$ may be determined from the supercuspidal support. We note that all the remaining possibilities except $\pi_{4}$ would require $m_{T}(\rho, a-2) \geq 2$ to occur, which is not the case. For $\pi_{4}$, Proposition 2.4.1 of [J7 tells us $m_{T_{3}}(\rho, a)=1$ and $m_{T_{3}}(\rho, a-2)=0$. Thus, by Proposition 6.1, we have $\pi_{4}=\nu^{\frac{-a+1}{2}} \rho \rtimes T_{3}$ (irreducible), from which we see $f_{\pi_{4}}\left(\nu^{\frac{a-1}{2}} \rho\right)=2$, too large for it to be a subquotient of $\nu^{\frac{-a+1}{2}} \rho \rtimes L\left(\nu^{\frac{-a+1}{2}} \rho ; T\right)$. The needed irreducibility and the proposition follow.

\section{REMARKS ON $x=0$}

In this section, we discuss the problems which arise when $x=0$.

The first issue which arises is that the algorithms for calculating $\mu_{\left\{\nu^{x} \rho\right\}}^{*}(\pi)$ need not work when $x=0$. To see this, consider the example of

$$
\pi=L\left(\nu^{-1} \rho, \nu^{-1} \rho, \rho \rtimes T_{i}(\rho ; \sigma)\right)
$$

in the case where $\operatorname{red}(\rho ; \sigma)=0$ and $\rho \rtimes \sigma \cong T_{1}(\rho ; \sigma) \oplus T_{-1}(\rho ; \sigma)$. Attempting the algorithm from section 3.3 would give $f_{\pi}(\rho)=0$. However, as

$$
\pi \hookrightarrow \zeta\left(\left[\nu^{-1} \rho, \nu \rho\right]\right) \rtimes T_{i}(\rho ; \sigma) \hookrightarrow \zeta\left(\left[\nu^{-1} \rho, \nu \rho\right]\right) \times \rho \rtimes \sigma \cong \rho \times \zeta\left(\left[\nu^{-1} \rho, \nu \rho\right]\right) \rtimes \sigma,
$$

Frobenius reciprocity tells us $f_{\pi}(\rho) \neq 0$.

Fortunately, this is not a major issue as we do not need to be able to calculate $\mu_{\{\rho\}}^{*}(\pi)$ for general $\pi$, only for those $\pi$ when there is no other choice, i.e., when 
$f_{\pi}\left(\nu^{x} \rho\right)=0$ for all $x \neq 0$. In that case, an easy variation of the previous algorithm applies. Write $\pi=L(\Delta ; T)$ and $\mu_{\{\rho\}}^{*}(T)=\underbrace{\rho \times \cdots \times \rho}_{f} \otimes T^{\prime}$ (noting that $T^{\prime}$ must be tempered and is given in Theorem 3.1). Further, note that any generalized Steinberg $\delta\left(\left[\nu^{a} \rho, \nu^{b} \rho\right]\right)$ appearing in $\Delta$ has $a<0$ (by conditions on Langlands data) and $b \geq 0$ (or else $f_{\pi}\left(\nu^{x} \rho\right)>0$ for some $\left.x<0\right)$. In particular, this means $\rho \rtimes \delta\left(\left[\nu^{a} \rho, \nu^{b} \rho\right]\right.$ ) is irreducible; by Lemma 1.3.3 of [J5], this means $\rho \times \mathcal{L}(\Delta)$ is irreducible. Thus,

$$
\pi \hookrightarrow \mathcal{L}(\Delta) \rtimes T \hookrightarrow \mathcal{L}(\Delta) \times \underbrace{\rho \times \cdots \times \rho}_{f} \rtimes T^{\prime} \cong \underbrace{\rho \times \cdots \times \rho}_{f} \times \mathcal{L}(\Delta) \rtimes T^{\prime} .
$$

If we write

$$
m_{\{\rho\}}^{*}(\mathcal{L}(\Delta))=\underbrace{\rho \times \cdots \times \rho}_{f^{\prime}} \otimes \mathcal{L}\left(\Delta^{\prime}\right)
$$

we get

$$
\pi \hookrightarrow \underbrace{\rho \times \cdots \times \rho}_{f+f^{\prime}} \times \mathcal{L}\left(\Delta^{\prime}\right) \rtimes T^{\prime} .
$$

Now, it follows from Theorem 2.2.1 or [J5] that the representations appearing in $\Delta^{\prime}$ have negative central exponents, so by Lemma 2.4

$$
\mu_{\{\rho\}}^{*}(\pi)=\underbrace{\rho \times \cdots \times \rho}_{f+f^{\prime}} \otimes L\left(\Delta^{\prime} ; T^{\prime}\right) .
$$

Thus, it is not difficult to determine $\mu_{\{\rho\}}^{*}(\pi)$ for those cases we need.

Another issue is that the results of section 3.4 have not been done for the case $a=3$. While not trivial to obtain, a version of Theorem 3.3 should certainly be possible. Note that in the case $\operatorname{red}(\rho ; \sigma) \equiv \frac{1}{2} \bmod 1$, such a result is given in section 8 .

A more critical issue arises in (1.2). The first property there holds up to multiplicity (which is sufficient for our purposes); the second property holds as is. However the third does not-we can have $\pi_{1} \not \pi_{2}$ with $\mu_{\{\rho\}}^{*}\left(\pi_{1}\right)=\mu_{\{\rho\}}^{*}\left(\pi_{2}\right)$. The simplest example is when $\operatorname{red}(\rho ; \sigma)=0$ and $\pi_{i}=T_{i}(\rho ; \sigma)$, but it is not hard to find less trivial examples. Again, we do not need the general case - it would be enough to have a result for $\pi$ having $f_{\pi}\left(\nu^{x} \rho\right)=0$ for all $x \neq 0$. We still have $\pi_{i}=T_{i}(\rho ; \sigma)$ as a counterexample, but nontrivial counterexamples are much rarer. The proposition below limits the possibilities.

Proposition 7.1. Suppose $\pi=L(\Delta ; T)$ satisfies

$$
\mu_{\left\{\nu^{x} \rho\right\}}^{*}(\pi)=0 \text { if } x \neq 0 .
$$

Write $\mu_{\{\rho\}}^{*}(\pi)=\underbrace{(\rho \times \cdots \times \rho)}_{f_{\pi}} \otimes \theta$ up to multiplicity. Suppose $\pi^{\prime}$ is an irreducible representation with $\mu_{\{\rho\}}^{*}\left(\pi^{\prime}\right)=\underbrace{(\rho \times \cdots \times \rho)}_{f_{\pi}} \otimes \theta$ up to multiplicity and also satisfies equation (7.1).

(1) If $m_{T}(\rho, 1)=0$ or is odd, then $\pi^{\prime}=\pi$.

(2) If $m_{T}(\rho, 1)$ is positive and even, then $\pi^{\prime}=\pi$ or $\pi^{*}$, where $\pi^{*}=L\left(\Delta ; T^{*}\right)$ and $T^{*}$ has data matching that of $T$ except $\varepsilon_{T^{*}}(\rho, 1)=-\varepsilon_{T}(\rho, 1)$. 
Proof. Write $\pi=L(\Delta ; T)=L\left(\delta\left(\left[\nu^{-b_{1}} \rho, \nu^{a_{1}} \rho\right]\right), \ldots,\left(\delta\left(\left[\nu^{-b_{k}} \rho, \nu^{a_{k}} \rho\right]\right) ; T\right)\right.$. We may assume that $a_{1} \leq a_{2} \leq \cdots \leq a_{k}$ as this reordering can be effected by commuting representations which induce irreducibly (see section 1.2 of [J5]). Further, we must have $a_{1}=0$ as long as $k>0$, i.e., $\pi$ nontempered. As the claim is easier when $k=0$, we assume $k>0$ below. In particular, this means there is some $\ell$ such that $a_{\ell}=0$ and $a_{\ell+1}>0$ (possibly $\ell=k$ ).

Write $\mu_{\{\rho\}}^{*}(T)=\underbrace{(\rho \times \cdots \times \rho)}_{f_{T}} \otimes T_{0}$, up to multiplicity. Since $a_{i}, b_{i} \geq 0$ for all $i$, we have $\rho \times \delta\left(\left[\nu^{-b_{i}} \rho, \nu^{a_{i}} \rho\right]\right) \cong \delta\left(\left[\nu^{-b_{i}} \rho, \nu^{a_{i}} \rho\right]\right) \times \rho$ (irreducible) for all $i$. Thus,

$$
\begin{aligned}
& \pi \hookrightarrow \delta\left(\left[\nu^{-b_{1}} \rho, \nu^{a_{1}} \rho\right]\right) \times \cdots \times \delta\left(\left[\nu^{-b_{k}} \rho, \nu^{a_{k}} \rho\right]\right) \times \underbrace{(\rho \times \cdots \times \rho)}_{f_{T}} \rtimes T_{0} \\
& \cong \underbrace{(\rho \times \cdots \times \rho)}_{f_{T}} \times \delta\left(\left[\nu^{-b_{1}} \rho, \rho\right]\right) \times \cdots \times \delta\left(\left[\nu^{-b_{\ell}} \rho, \rho\right]\right) \times \delta\left(\left[\nu^{-b_{\ell+1}} \rho, \nu^{a_{\ell+1}} \rho\right]\right) \\
& \times \cdots \times \delta\left(\left[\nu^{-b_{k}} \rho, \nu^{a_{k}} \rho\right]\right) \rtimes T_{0} \\
& \hookrightarrow \underbrace{(\rho \times \cdots \times \rho)}_{\ell+f_{T}} \times \delta\left(\left[\nu^{-b_{1}} \rho, \nu^{-1} \rho\right]\right) \times \cdots \times \delta\left(\left[\nu^{-b_{\ell}} \rho, \nu^{-1} \rho\right]\right) \times \delta\left(\left[\nu^{-b_{\ell+1}} \rho, \nu^{a_{\ell+1}} \rho\right]\right) \\
& \times \cdots \times \delta\left(\left[\nu^{-b_{k}} \rho, \nu^{a_{k}} \rho\right]\right) \rtimes T_{0} .
\end{aligned}
$$

From this embedding, we immediately see that $f_{\pi}=\ell+f_{T}$. It then follows from Lemma 2.4 that up to multiplicity,

$$
\begin{aligned}
\mu_{\{\rho\}}^{*}(\pi) & \underbrace{(\rho \times \cdots \times \rho)}_{\ell+f_{T}} \otimes L\left(\delta\left(\left[\nu^{-b_{1}} \rho, \nu^{-1} \rho\right]\right), \ldots, \delta\left(\left[\nu^{-b_{\ell}} \rho, \nu^{-1} \rho\right]\right), \delta\left(\left[\nu^{-b_{\ell+1}} \rho, \nu^{a_{\ell+1}} \rho\right]\right),\right. \\
& =\underbrace{(\rho \times \cdots \times \rho)}_{\ell+f_{T}} \otimes \theta, \delta\left(\left[\nu^{-b_{k}} \rho, \nu^{a_{k}} \rho\right]\right) ; T_{0})
\end{aligned}
$$

(defining $\theta$ ).

Now, suppose $\pi^{\prime}$ is an irreducible representation satisfying equation (7.1) and having $\mu_{\{\rho\}}^{*}\left(\pi^{\prime}\right)=\underbrace{(\rho \times \cdots \times \rho)}_{\ell+f_{T}} \otimes \theta$ up to multiplicity. Then,

$$
\begin{gathered}
\pi^{\prime} \hookrightarrow \underbrace{(\rho \times \cdots \times \rho)}_{\ell+f_{T}} \times \mathcal{L}\left(\delta\left(\left[\nu^{-b_{1}} \rho, \nu^{-1} \rho\right]\right), \ldots, \delta\left(\left[\nu^{-b_{\ell}} \rho, \nu^{-1} \rho\right]\right), \delta\left(\left[\nu^{-b_{\ell+1}} \rho, \nu^{a_{\ell+1}} \rho\right]\right),\right. \\
\left.\left.\ldots, \delta\left(\left[\nu^{-b_{k}} \rho, \nu^{a_{k}} \rho\right]\right)\right) \rtimes T_{0}\right) \\
\Downarrow \hookrightarrow \underbrace{(\rho \times \cdots \times \rho)}_{f_{T}} \times \lambda \rtimes T_{0}
\end{gathered}
$$

for some irreducible

$$
\begin{aligned}
\lambda & \leq \underbrace{(\rho \times \cdots \times \rho)}_{\ell} \\
& \times \mathcal{L}\left(\delta\left(\left[\nu^{-b_{1}} \rho, \nu^{-1} \rho\right]\right), \ldots, \delta\left(\left[\nu^{-b_{\ell}} \rho, \nu^{-1} \rho\right]\right), \delta\left(\left[\nu^{-b_{\ell+1}} \rho, \nu^{a_{\ell+1}} \rho\right]\right), \ldots, \delta\left(\left[\nu^{-b_{k}} \rho, \nu^{a_{k}} \rho\right]\right)\right)
\end{aligned}
$$


(Lemma 2.2). As $f_{\lambda}(\rho)=\ell$ (since $f_{\lambda}(\rho)<\ell$ would imply $f_{\pi^{\prime}}(\rho)<f_{T}+\ell$ ), we may conclude that

$$
\lambda=\mathcal{L}\left(\delta\left(\left[\nu^{-b_{1}} \rho, \rho\right]\right), \ldots, \delta\left(\left[\nu^{-b_{\ell}} \rho, \rho\right]\right), \delta\left(\left[\nu^{-b_{\ell+1}} \rho, \nu^{a_{\ell+1}} \rho\right]\right), \ldots, \delta\left(\left[\nu^{-b_{k}} \rho, \nu^{a_{k}} \rho\right]\right)\right) .
$$

Then,

$$
\begin{array}{r}
\pi^{\prime} \hookrightarrow \underbrace{(\rho \times \cdots \times \rho)}_{f_{T}} \times \mathcal{L}\left(\delta\left(\left[\nu^{-b_{1}} \rho, \rho\right]\right), \ldots, \delta\left(\left[\nu^{-b_{\ell}} \rho, \rho\right]\right), \delta\left(\left[\nu^{-b_{\ell+1}} \rho, \nu^{a_{\ell+1}} \rho\right]\right),\right. \\
\left.\ldots, \delta\left(\left[\nu^{-b_{k}} \rho, \nu^{a_{k}} \rho\right]\right)\right) \rtimes T_{0} \\
\cong \mathcal{L}\left(\delta\left(\left[\nu^{-b_{1}} \rho, \rho\right]\right), \ldots, \delta\left(\left[\nu^{-b_{\ell}} \rho, \rho\right]\right), \delta\left(\left[\nu^{-b_{\ell+1}} \rho, \nu^{a_{\ell+1}} \rho\right]\right), \ldots, \delta\left(\left[\nu^{-b_{k}} \rho, \nu^{a_{k}} \rho\right]\right)\right) \\
\times \underbrace{(\rho \times \cdots \times \rho)}_{f_{T}} \rtimes T_{0},
\end{array}
$$

noting the irreducibility of

$$
\rho \times \mathcal{L}\left(\delta\left(\left[\nu^{-b_{1}} \rho, \rho\right]\right), \ldots, \delta\left(\left[\nu^{-b_{\ell}} \rho, \rho\right]\right), \delta\left(\left[\nu^{-b_{\ell+1}} \rho, \nu^{a_{\ell+1}} \rho\right]\right), \ldots, \delta\left(\left[\nu^{-b_{k}} \rho, \nu^{a_{k}} \rho\right]\right)\right)
$$

follows from that of $\rho \times \delta\left(\left[\nu^{-b_{i}} \rho, \nu^{a_{i}} \rho\right]\right)$ by Lemma 1.3.3 of [J5]. It then follows from the Langlands classification that

$$
\pi^{\prime}=L\left(\delta\left(\left[\nu^{-b_{1}} \rho, \rho\right]\right), \ldots, \delta\left(\left[\nu^{-b_{\ell}} \rho, \rho\right]\right), \delta\left(\left[\nu^{-b_{\ell+1}} \rho, \nu^{a_{\ell+1}} \rho\right]\right), \ldots, \delta\left(\left[\nu^{-b_{k}} \rho, \nu^{a_{k}} \rho\right]\right) ; T^{\prime}\right)
$$

for some component $T^{\prime} \leq \underbrace{(\rho \times \cdots \times \rho)}_{f_{T}} \rtimes T_{0}$. If $f_{T}=0$ (corresponding to $m(\rho, 1) \leq$

1) or $m_{T_{0}}(\rho, 1)=1$ (corresponding to $m_{T}(\rho, 1)$ positive and odd), we have $T^{\prime}=$ $\underbrace{(\rho \times \cdots \times \rho)}_{f_{T}} \rtimes T_{0}=T$ irreducible. If neither of these holds, $\underbrace{(\rho \times \cdots \times \rho)}_{f_{T}} \rtimes T_{0} \cong$ $T \oplus T^{*}$, so $T^{\prime}=T$ or $T^{*}$. The proposition follows.

We emphasize that not every pair $\pi, \pi^{*}$ as in (2) of the proposition necessarily have $\mu_{\{\rho\}}^{*}(\pi)=\mu_{\{\rho\}}^{*}\left(\pi^{*}\right)$, and in practice, it seems most do not. So, even in the integral case, the duality algorithm from section 1-coupled with the observation that if $\operatorname{red}(\rho ; \sigma)=0$ (resp., $\operatorname{red}(\rho ; \sigma) \neq 0)$, the dual of (the irreducible representation) $\pi_{i}=\rho \times \cdots \times \rho \rtimes T_{i}(\rho ; \sigma)$ (resp., $\left.\pi=\rho \times \cdots \times \rho \rtimes \sigma\right)$ is $\pi_{-i}$ (resp., $\pi$ ) - is sometimes enough to calculate the dual.

\section{THE CASE $\pi \in R\left(\left(\rho, x_{0}\right) ; \sigma\right)$ WITH $x_{0} \neq \frac{1}{2}$}

Throughout most of this paper, we have focused on the most interesting and difficult case -when $\pi \in R\left(\left(\rho, \frac{1}{2}\right) ; \sigma\right)$ (so $\left.x \equiv \operatorname{red}(\rho ; \sigma) \bmod 1\right)$. In this section, we take up what happens when the supercuspidal support of $\pi$ is in $\left\{\nu^{x} \rho, \nu^{-x} \rho\right\}_{x \in x_{0}+\mathbb{Z}}$ where $0 \leq x_{0}<\frac{1}{2}$.

First, we note that if $x_{0} \notin \frac{1}{2} \mathbb{Z}$, the situation is quite straightforward- $\mu_{\{\rho\}}^{*}$ does not arise in the duality algorithm and the algorithms of sections 3.2 and 3.3 allow us to calculate $\mu_{\left\{\nu^{x} \rho\right\}}^{*}(\pi)$; the situation covered by results of section 3.4 does not occur. The only case requiring additional work is that when $x_{0}=0$. Thus we assume $x_{0}=0$ in the remainder of the section.

The first issues that need to be addressed in this case are those connected to $\mu_{\{\rho\}}^{*}$. As noted in section 7 we need only calculate $\nu_{\{\rho\}}^{*}(\pi)$ when there are no other options, i.e., when $f_{\pi}\left(\nu^{x} \rho\right)=0$ for all $x \neq 0$. In this case, the algorithm given in the beginning of section 7 allows us to calculate $\mu_{\{\rho\}}^{*}(\pi)$. There is also the issue that one can have $\mu_{\{\rho\}}^{*}\left(\pi_{1}\right)=\mu_{\{\rho\}}^{*}\left(\pi_{2}\right)$ for $\pi_{1} \neq \pi_{2}$ (e.g., $\pi_{1}=L\left(\delta\left(\left[\nu^{-1} \rho, \rho\right]\right) ; \sigma\right)$ and 
$\left.\pi_{2}=L\left(\nu^{-1} \rho ; \rho \rtimes \sigma\right)\right)$. Proposition 7.1 tells us this cannot happen here for $\pi_{1}$ and $\pi_{2}$ both having $f_{\pi_{i}}\left(\nu^{x} \rho\right)=0$ for all $x \neq 0$. Therefore, since we use $\mu_{\{\rho\}}^{*}$ only when there are no other options, if we arrive at $\mu^{*}(\hat{\pi})=(\rho)^{k} \otimes \lambda$ in the duality algorithm and there are two possibilities for $\hat{\pi}$, we choose the one which has $f\left(\nu^{x} \rho\right)=0$ for all $x \neq 0$ (noting that this property is clearly preserved under duality).

The results of section 3.4 are more delicate in their dependence on reducibility values, etc. The situation covered by section 3.4 can occur in this case, and the results there must be adapted to this case. Lemma 8.3. Lemma 8.4 and Proposition 8.5 are the analogue of Theorem 3.3 -here for the case $a=3$ - while Proposition 8.6 is the analogue of Proposition 3.4. The algorithm at the end of section 3.4 then works as is for the general case.

We begin with some irreducibility results needed later.

Lemma 8.1. The following representations are irreducible:

(1) $\nu^{-1} \rho \times \mathcal{L}\left(\delta\left(\left[\nu^{-1} \rho, \rho\right]\right), \delta([\rho, \nu \rho])\right)$ and $\nu \rho \times \mathcal{L}\left(\delta\left(\left[\nu^{-1} \rho, \rho\right]\right), \delta([\rho, \nu \rho])\right)$.

(2) $\delta\left(\left[\nu^{-1} \rho, \rho\right]\right) \times \mathcal{L}\left(\delta\left(\left[\nu^{-1} \rho, \rho\right]\right), \delta([\rho, \nu \rho])\right)$ and

$$
\delta([\rho, \nu \rho]) \times \mathcal{L}\left(\delta\left(\left[\nu^{-1} \rho, \rho\right]\right), \delta([\rho, \nu \rho])\right) .
$$

(3) $\zeta\left(\left[\nu^{-1} \rho, \rho\right]\right) \times \mathcal{L}\left(\nu^{-1} \rho, \delta([\rho, \nu \rho])\right)$.

Proof. These may be proven using arguments like that in the proof of Lemma 3.6 of [J8] or via a Jacquet module analysis like that in B-J1 (but easier as only general linear groups are involved).

Lemma 8.2. $\delta\left(\left[\nu^{-1} \rho, \rho\right]\right) \rtimes T$ is irreducible.

Proof. First, we claim $\delta\left(\left[\nu^{-1} \rho, \rho\right]\right) \rtimes \sigma$ is irreducible. Note that for some of the groups in question, this is done in Proposition 6.3 of [T2. More generally, were $\delta\left(\left[\nu^{-1} \rho, \rho\right]\right) \rtimes \sigma$ reducible, the irreducible subquotients would have to have minimal Jacquet modules $r_{M, G}\left(\pi_{1}\right)=\rho \otimes \nu^{-1} \rho \otimes \sigma+\rho \otimes \nu \rho \otimes \sigma$ and $r_{M, G}\left(\pi_{2}\right)=2 \cdot \nu \rho \otimes \rho \otimes \sigma$. In particular, $\pi_{2}$ would be square-integrable by the Casselman criterion. However, by [Mœ2, [M-T], no such discrete series exists. Thus, $\delta\left(\left[\nu^{-1} \rho, \rho\right]\right) \rtimes \sigma$ is irreducible.

Now, observe that as $x_{0} \not \equiv \operatorname{red}(\rho ; \sigma) \bmod 1, R\left(\left(\rho, x_{0}\right) ; \sigma\right)$ has no nontrivial elliptic tempered representations. In particular, we have $T$ irreducibly induced as in (2.3) with $T_{\text {ell }}=\sigma$. Let $\pi=L\left(\delta\left(\left[\nu^{-1} \rho, \rho\right]\right) ; T\right)$. Then,

$$
\pi \hookrightarrow \delta\left(\left[\nu^{-1} \rho, \rho\right]\right) \rtimes T \hookrightarrow \delta\left(\left[\nu^{-1} \rho, \rho\right]\right) \times \Lambda \rtimes \sigma,
$$

where $\Lambda$ is a product of generalized Steinberg representations arising from (2.3). Noting the irreducibility of $\delta\left(\left[\nu^{-1} \rho, \rho\right]\right) \times \Lambda, \delta([\rho, \nu \rho]) \times \Lambda$, and $\delta\left(\left[\nu^{-1} \rho, \rho\right]\right) \rtimes \sigma$, a commuting/inverting argument tells us

$$
\begin{gathered}
\pi \hookrightarrow \delta\left(\left[\nu^{-1} \rho, \rho\right]\right) \times \Lambda \rtimes \sigma \\
\cong \Lambda \times \delta\left(\left[\nu^{-1} \rho, \rho\right]\right) \rtimes \sigma \cong \Lambda \times \delta([\rho, \nu \rho]) \rtimes \sigma \cong \delta([\rho, \nu \rho]) \times \Lambda \rtimes \sigma \\
\Downarrow(\text { Lemma } 2.2) \\
\pi \hookrightarrow \delta([\rho, \nu \rho]) \rtimes T^{\prime}
\end{gathered}
$$

for some irreducible $T^{\prime} \leq \Lambda \rtimes \sigma$. We claim $T^{\prime}=T$. Now,

$$
\mu^{*}(\pi) \leq \mu^{*}\left(\delta([\rho, \nu \rho]) \rtimes T^{\prime}\right)=\mu^{*}\left(\delta\left(\left[\nu^{-1} \rho, \rho\right]\right) \rtimes T^{\prime}\right) ;
$$

by Proposition 5.3 of [B-J3], $\delta\left(\left[\nu^{-1} \rho, \rho\right]\right) \otimes T^{\prime}$ is the unique Jacquet module subquotient having its central character. Consequently $\delta\left(\left[\nu^{-1} \rho, \rho\right]\right) \otimes T$ must be 
$\delta\left(\left[\nu^{-1} \rho, \rho\right]\right) \otimes T^{\prime}$, implying $T^{\prime}=T$, as claimed. This tells us $\pi$ appears as both the Langlands quotient in $\delta([\rho, \nu \rho]) \rtimes T$ and as a subrepresentation. This contradicts multiplicity one unless we have irreducibility.

Lemma 8.3. For $\pi=L\left(\delta\left(\left[\nu^{-1} \rho, \rho\right]\right)^{\ell} ; T\right)$, write $\mu^{*}(T)=(\nu \rho)^{f_{T}} \otimes T^{\prime}$. We have

$$
\mu_{\{\nu \rho\}}^{*}(\pi)=\left\{\begin{array}{l}
(\nu \rho)^{f_{T}+1} \otimes L\left(\delta\left(\left[\nu^{-1} \rho, \rho\right]\right)^{\ell-1} ; \rho \rtimes T^{\prime}\right) \text { if } \ell \text { odd }, \\
(\nu \rho)^{f_{T}} \otimes L\left(\delta\left(\left[\nu^{-1} \rho, \rho\right]\right)^{\ell} ; T^{\prime}\right) \text { if } \ell \text { even. }
\end{array}\right.
$$

Proof. The proof is similar to that of Proposition 8.5 below; we forgo the details here.

Lemma 8.4. For $\pi=L\left(\left(\nu^{-1} \rho\right)^{k} ; T\right)$, write $\mu^{*}(T)=(\nu \rho)^{f_{T}} \otimes T^{\prime}$. If $T=(\rho)^{k_{0}} \rtimes T_{0}$, with $f_{T_{0}}(\rho)=0$, we have

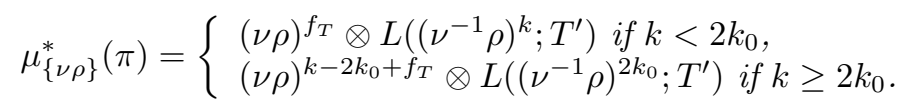

Proof. The proof is done in three cases: $k \leq k_{0}, k_{0}<k<2 k_{0}$, and $2 k_{0} \leq k$. The arguments for all three cases are similar; we do only the second case in detail.

We have

$$
\begin{aligned}
& \pi \hookrightarrow\left(\nu^{-1} \rho\right)^{k} \times(\rho)^{k_{0}} \rtimes T_{0} \\
& \Downarrow(\text { Lemma } 2.2 \text { and Lemma 1.3.5 of [J5] }) \\
& \pi \hookrightarrow\left(\nu^{-1} \rho\right)^{k-k_{0}} \times \zeta\left(\left[\nu^{-1} \rho, \rho\right]\right)^{k^{\prime}} \times \delta\left(\left[\nu^{-1} \rho, \rho\right]\right)^{k_{0}-k^{\prime}} \rtimes T_{0}
\end{aligned}
$$

for some $k^{\prime}$. We note that in the embedding above, we get $f_{\pi}\left(\nu^{-1} \rho\right)=k-k_{0}+k^{\prime}$, hence must have $k^{\prime}=k_{0}$. Thus,

$$
\begin{aligned}
\pi & \hookrightarrow\left(\nu^{-1} \rho\right)^{k-k_{0}} \times \zeta\left(\left[\nu^{-1} \rho, \rho\right]\right)^{k_{0}} \rtimes T_{0} \\
& \cong \zeta\left(\left[\nu^{-1} \rho, \rho\right]\right)^{k_{0}} \times\left(\nu^{-1} \rho\right)^{k-k_{0}} \rtimes T_{0} \\
& \cong \zeta\left(\left[\nu^{-1} \rho, \rho\right]\right)^{k_{0}} \times(\nu \rho)^{k-k_{0}} \rtimes T_{0} .
\end{aligned}
$$

By Lemma 2.2,

$$
\begin{gathered}
\pi \hookrightarrow \zeta\left(\left[\nu^{-1} \rho, \rho\right]\right)^{k_{0}-1} \times \mathcal{L}\left(\nu^{-1} \rho, \delta([\rho, \nu \rho])\right) \times(\nu \rho)^{k-k_{0}-1} \rtimes T_{0} \\
\pi \hookrightarrow \zeta\left(\left[\nu^{-1} \rho, \rho\right]\right)^{k_{0}-1} \times \zeta\left(\left[\nu^{-1} \rho, \nu \rho\right]\right) \times(\nu \rho)^{k-k_{0}-1} \rtimes T_{0} .
\end{gathered}
$$

Observe that the former would imply $f_{\pi}\left(\nu^{-1} \rho\right) \leq\left(k_{0}-1\right)+1+\left(k-k_{0}-1\right)=k-1$. Thus it must be the latter. Therefore,

$$
\pi \hookrightarrow \zeta\left(\left[\nu^{-1} \rho, \nu \rho\right]\right) \times \zeta\left(\left[\nu^{-1} \rho, \rho\right]\right)^{k_{0}-1} \times(\nu \rho)^{k-k_{0}-1} \rtimes T_{0} .
$$

Iterating this argument, we eventually arrive at

$$
\pi \hookrightarrow \zeta\left(\left[\nu^{-1} \rho, \nu \rho\right]\right)^{k-k_{0}} \times \zeta\left(\left[\nu^{-1} \rho, \rho\right]\right)^{2 k_{0}-k} \rtimes T_{0} .
$$

The embedding

$$
\pi \hookrightarrow\left(\nu^{-1} \rho\right)^{k} \times(\nu \rho)^{f_{T}} \rtimes T^{\prime} \cong(\nu \rho)^{f_{T}} \times\left(\nu^{-1} \rho\right)^{k} \rtimes T^{\prime}
$$

tells us $f_{\pi}(\nu \rho) \geq f_{T}$. Now, write $\mu_{\{\nu \rho\}}^{*}\left(T_{0}\right)=(\nu \rho)^{f_{T}} \otimes T_{0}^{\prime}$, noting that $f_{T_{0}}(\nu \rho)=f_{T}$ by Theorem 3.1 Then,

$$
\begin{gathered}
\pi \hookrightarrow \zeta\left(\left[\nu^{-1} \rho, \nu \rho\right]\right)^{k-k_{0}} \times \zeta\left(\left[\nu^{-1} \rho, \rho\right]\right)^{2 k_{0}-k} \times(\nu \rho)^{f_{T}} \rtimes T_{0}^{\prime} \\
\Downarrow(\operatorname{Lemma}[2.2) \\
\pi \hookrightarrow \zeta\left(\left[\nu^{-1} \rho, \nu \rho\right]\right)^{k-k_{0}} \times \zeta\left(\left[\nu^{-1} \rho, \rho\right]\right)^{2 k_{0}-k-1} \times \zeta\left(\left[\nu^{-1} \rho, \nu \rho\right]\right) \times(\nu \rho)^{f_{T}-1} \rtimes T_{0}^{\prime} \\
\text { or } \hookrightarrow \zeta\left(\left[\nu^{-1} \rho, \nu \rho\right]\right)^{k-k_{0}} \times \zeta\left(\left[\nu^{-1} \rho, \rho\right]\right)^{2 k_{0}-k-1} \times \mathcal{L}\left(\nu^{-1} \rho, \delta([\rho, \nu \rho])\right) \times(\nu \rho)^{f_{T}-1} \rtimes T_{0}^{\prime} .
\end{gathered}
$$


As the former would imply $f_{\pi}(\nu \rho) \leq f_{T}-1$, it must be the latter. Then, by Lemma 8.1 and the embedding $\mathcal{L}\left(\nu^{-1} \rho, \delta([\rho, \nu \rho])\right) \hookrightarrow \nu \rho \times \zeta\left(\left[\nu^{-1} \rho, \rho\right]\right)$,

$$
\begin{aligned}
\pi & \hookrightarrow \zeta\left(\left[\nu^{-1} \rho, \nu \rho\right]\right)^{k-k_{0}} \times \zeta\left(\left[\nu^{-1} \rho, \rho\right]\right)^{2 k_{0}-k-1} \times \mathcal{L}\left(\nu^{-1} \rho, \delta([\rho, \nu \rho])\right) \times(\nu \rho)^{f_{T}-1} \rtimes T_{0}^{\prime} \\
& \cong \zeta\left(\left[\nu^{-1} \rho, \nu \rho\right]\right)^{k-k_{0}} \times \mathcal{L}\left(\nu^{-1} \rho, \delta([\rho, \nu \rho])\right) \times \zeta\left(\left[\nu^{-1} \rho, \rho\right]\right)^{2 k_{0}-k-1} \times(\nu \rho)^{f_{T}-1} \rtimes T_{0}^{\prime} \\
& \hookrightarrow \zeta\left(\left[\nu^{-1} \rho, \nu \rho\right]\right)^{k-k_{0}} \times\left(\nu \rho \times \zeta\left(\left[\nu^{-1} \rho, \rho\right]\right)\right) \times \zeta\left(\left[\nu^{-1} \rho, \rho\right]\right)^{2 k_{0}-k-1} \times(\nu \rho)^{f_{T}-1} \rtimes T_{0}^{\prime} \\
& \cong \nu \rho \times \zeta\left(\left[\nu^{-1} \rho, \nu \rho\right]\right)^{k-k_{0}} \times \zeta\left(\left[\nu^{-1} \rho, \rho\right]\right)^{2 k_{0}-k} \times(\nu \rho)^{f_{T}-1} \rtimes T_{0}^{\prime} .
\end{aligned}
$$

Iterating this argument, we eventually arrive at

$$
\pi \hookrightarrow(\nu \rho)^{f_{T}} \times \zeta\left(\left[\nu^{-1} \rho, \nu \rho\right]\right)^{k-k_{0}} \times \zeta\left(\left[\nu^{-1} \rho, \rho\right]\right)^{2 k_{0}-k} \rtimes T_{0}^{\prime} .
$$

Note that this tells us $f_{\pi}(\nu \rho)=f_{T}$. By (8.1) and Lemma 2.4, it follows that

$$
\mu_{\{\nu \rho\}}^{*}(\pi)=(\nu \rho)^{f_{T}} \otimes L\left(\left(\nu^{-1} \rho\right)^{k} ; T^{\prime}\right),
$$

as needed.

Proposition 8.5. Let $\pi=L\left(\left(\nu^{-1}\right)^{k}, \delta\left(\left[\nu^{-1} \rho, \rho\right]\right)^{\ell} ; T\right)$ with $k, \ell>0$. Let

$$
\lambda=\left\{\begin{array}{l}
L\left(\left(\nu^{-1}\right)^{k-1} ; T\right) \text { if } \ell \text { odd }, \\
L\left(\left(\nu^{-1}\right)^{k} ; T\right) \text { if } \ell \text { even, }
\end{array}\right.
$$

and write $\mu_{\{\nu \rho\}}^{*}(\lambda)=(\nu \rho)^{f_{\lambda}} \otimes L\left(\left(\nu^{-1} \rho\right)^{k^{*}} ; T^{*}\right)$ (Lemma 8.4). Then,

$$
\mu_{\{\nu \rho\}}^{*}(\pi)=\left\{\begin{array}{l}
(\nu \rho)^{f_{\lambda}+1} \otimes L\left(\left(\nu^{-1}\right)^{k^{*}+1}, \delta\left(\left[\nu^{-1} \rho, \rho\right]\right)^{\ell-1} ; \rho \rtimes T^{*}\right) \text { if } \ell \text { odd }, \\
(\nu \rho)^{f_{\lambda}} \otimes L\left(\left(\nu^{-1}\right)^{k^{*}}, \delta\left(\left[\nu^{-1} \rho, \rho\right]\right)^{\ell} ; T^{*}\right) \text { if } \ell \text { even. }
\end{array}\right.
$$

Proof. First, suppose $\ell$ is odd and write $\ell=2 j+1$ with $j \geq 0$. Noting that $\delta\left(\left[\nu^{-1} \rho, \rho\right]\right) \rtimes T$ is irreducible (Lemma 8.2),

$$
\begin{gathered}
\pi \hookrightarrow\left(\nu^{-1} \rho\right)^{k} \times \delta\left(\left[\nu^{-1} \rho, \rho\right]\right)^{2 j+1} \rtimes T \cong\left(\nu^{-1} \rho\right)^{k} \times \delta\left(\left[\nu^{-1} \rho, \rho\right]\right)^{2 j} \times \delta([\rho, \nu \rho]) \rtimes T \\
\Downarrow(\operatorname{Lemma} 2.2) \\
\pi \hookrightarrow\left(\nu^{-1} \rho\right)^{k} \times \delta\left(\left[\nu^{-1} \rho, \rho\right]\right)^{2 j-1} \times \mathcal{L}\left(\delta\left(\left[\nu^{-1} \rho, \rho\right]\right), \delta([\rho, \nu \rho])\right) \rtimes T \\
\text { or } \\
\left(\nu^{-1} \rho\right)^{k} \times \delta\left(\left[\nu^{-1} \rho, \rho\right]\right)^{2 j-1} \times \delta\left(\left[\nu^{-1} \rho, \nu \rho\right]\right) \times \rho \rtimes T .
\end{gathered}
$$

The latter would imply $\pi=L\left(\left(\nu^{-1} \rho\right)^{k}, \delta\left(\left[\nu^{-1} \rho, \rho\right]\right)^{2 j-1} ; \delta\left(\left[\nu^{-1} \rho, \nu \rho\right]\right) \times \rho \rtimes T\right)$ by the Langlands classification, so it must be the former. Noting the irreducibility of $\delta\left(\left[\nu^{-1} \rho, \rho\right]\right) \times \mathcal{L}\left(\delta\left(\left[\nu^{-1} \rho, \rho\right]\right), \delta([\rho, \nu \rho])\right)($ Lemma 8.1)

$$
\begin{aligned}
& \pi \hookrightarrow\left(\nu^{-1} \rho\right)^{k} \times \mathcal{L}\left(\delta\left(\left[\nu^{-1} \rho, \rho\right]\right), \delta([\rho, \nu \rho])\right) \times \delta\left(\left[\nu^{-1} \rho, \rho\right]\right)^{2 j-1} \rtimes T \\
& \Downarrow \text { (Lemma } 8.2 \text { and Lemma 2.2) } \\
& \pi \hookrightarrow\left(\nu^{-1} \rho\right)^{k} \times \mathcal{L}\left(\delta\left(\left[\nu^{-1} \rho, \rho\right]\right), \delta([\rho, \nu \rho])\right) \\
& \times \delta\left(\left[\nu^{-1} \rho, \rho\right]\right)^{2 j-3} \times \mathcal{L}\left(\delta\left(\left[\nu^{-1} \rho, \rho\right]\right), \delta([\rho, \nu \rho])\right) \rtimes T \\
& \text { or } \\
& \left(\nu^{-1} \rho\right)^{k} \times \mathcal{L}\left(\delta\left(\left[\nu^{-1} \rho, \rho\right]\right), \delta([\rho, \nu \rho])\right) \times \delta\left(\left[\nu^{-1} \rho, \rho\right]\right)^{2 j-3} \times \delta\left(\left[\nu^{-1} \rho, \nu \rho\right]\right) \times \rho \rtimes T .
\end{aligned}
$$

The latter would give

$$
\begin{aligned}
\pi \hookrightarrow\left(\nu^{-1} \rho\right)^{k} & \times \mathcal{L}\left(\delta\left(\left[\nu^{-1} \rho, \rho\right]\right), \delta([\rho, \nu \rho])\right) \\
& \times \delta\left(\left[\nu^{-1} \rho, \rho\right]\right)^{2 j-3} \times \delta\left(\left[\nu^{-1} \rho, \nu \rho\right]\right) \times \rho \rtimes T \\
\cong\left(\nu^{-1} \rho\right)^{k} & \times \delta\left(\left[\nu^{-1} \rho, \rho\right]\right)^{2 j-3} \\
& \times \mathcal{L}\left(\delta\left(\left[\nu^{-1} \rho, \rho\right]\right), \delta([\rho, \nu \rho])\right) \times \delta\left(\left[\nu^{-1} \rho, \nu \rho\right]\right) \times \rho \rtimes T \\
\hookrightarrow\left(\nu^{-1} \rho\right)^{k} & \times \delta\left(\left[\nu^{-1} \rho, \rho\right]\right)^{2 j-3} \\
& \times \delta\left(\left[\nu^{-1} \rho, \rho\right]\right) \times \delta([\rho, \nu \rho]) \rtimes\left(\delta\left(\left[\nu^{-1} \rho, \nu \rho\right]\right) \times \rho \rtimes T\right) \\
\cong\left(\nu^{-1} \rho\right)^{k} & \times \delta\left(\left[\nu^{-1} \rho, \rho\right]\right)^{2 j-3} \\
& \times \delta\left(\left[\nu^{-1} \rho, \rho\right]\right) \times \delta\left(\left[\nu^{-1} \rho, \rho\right]\right) \rtimes\left(\delta\left(\left[\nu^{-1} \rho, \nu \rho\right]\right) \times \rho \rtimes T\right) .
\end{aligned}
$$


By the Langlands classification, this would again imply

$$
\pi=L\left(\left(\nu^{-1} \rho\right)^{k}, \delta\left(\left[\nu^{-1} \rho, \rho\right]\right)^{2 j-1} ; \delta\left(\left[\nu^{-1} \rho, \nu \rho\right]\right) \times \rho \rtimes T\right),
$$

so again, it must be the former. Iterating, we eventually arrive at

$$
\pi \hookrightarrow\left(\nu^{-1} \rho\right)^{k} \times \delta\left(\left[\nu^{-1} \rho, \rho\right]\right) \times \mathcal{L}\left(\delta\left(\left[\nu^{-1} \rho, \rho\right]\right), \delta([\rho, \nu \rho])\right)^{j} \rtimes T .
$$

As $\delta\left(\left[\nu^{-1} \rho, \rho\right]\right) \times \mathcal{L}\left(\delta\left(\left[\nu^{-1} \rho, \rho\right]\right), \delta([\rho, \nu \rho])\right)$ and $\delta([\rho, \nu \rho]) \times \mathcal{L}\left(\delta\left(\left[\nu^{-1} \rho, \rho\right]\right), \delta([\rho, \nu \rho])\right)$ are irreducible (Lemma 8.1), we have

$$
\begin{aligned}
\pi & \hookrightarrow\left(\nu^{-1} \rho\right)^{k} \times \delta\left(\left[\nu^{-1} \rho, \rho\right]\right) \times \mathcal{L}\left(\delta\left(\left[\nu^{-1} \rho, \rho\right]\right), \delta([\rho, \nu \rho])\right)^{j} \rtimes T \\
& \cong\left(\nu^{-1} \rho\right)^{k} \times \mathcal{L}\left(\delta\left(\left[\nu^{-1} \rho, \rho\right]\right), \delta([\rho, \nu \rho])\right)^{j} \times \delta\left(\left[\nu^{-1} \rho, \rho\right]\right) \rtimes T \\
& \cong\left(\nu^{-1} \rho\right)^{k} \times \mathcal{L}\left(\delta\left(\left[\nu^{-1} \rho, \rho\right]\right), \delta([\rho, \nu \rho])\right)^{j} \times \delta([\rho, \nu \rho]) \rtimes T \\
& \cong\left(\nu^{-1} \rho\right)^{k} \times \delta([\rho, \nu \rho]) \times \mathcal{L}\left(\delta\left(\left[\nu^{-1} \rho, \rho\right]\right), \delta([\rho, \nu \rho])\right)^{j} \rtimes T .
\end{aligned}
$$

By Lemma 2.2, we have either

$$
\begin{gathered}
\pi \hookrightarrow\left(\nu^{-1} \rho\right)^{k-1} \times \mathcal{L}\left(\nu^{-1} \rho, \delta([\rho, \nu \rho])\right) \times \mathcal{L}\left(\delta\left(\left[\nu^{-1} \rho, \rho\right]\right), \delta([\rho, \nu \rho])\right)^{j} \rtimes T \\
\pi \hookrightarrow\left(\nu^{-1} \rho\right)^{k-1} \times \delta\left(\left[\nu^{-1} \rho, \nu \rho\right]\right) \times \mathcal{L}\left(\delta\left(\left[\nu^{-1} \rho, \rho\right]\right), \delta([\rho, \nu \rho])\right)^{j} \rtimes T .
\end{gathered}
$$

The latter would imply $f_{\pi}\left(\nu^{-1} \rho\right)=k-1$, so the former must hold. Noting the irreducibility of Lemma 3.6 of [J8] and Lemma 8.1] we then have

$$
\begin{aligned}
& \pi \hookrightarrow\left(\nu^{-1} \rho\right)^{k-1} \times \mathcal{L}\left(\nu^{-1} \rho, \delta([\rho, \nu \rho])\right) \times \mathcal{L}\left(\delta\left(\left[\nu^{-1} \rho, \rho\right]\right), \delta([\rho, \nu \rho])\right)^{j} \rtimes T \\
& \cong \mathcal{L}\left(\nu^{-1} \rho, \delta([\rho, \nu \rho])\right) \times \mathcal{L}\left(\delta\left(\left[\nu^{-1} \rho, \rho\right]\right), \delta([\rho, \nu \rho])\right)^{j} \times\left(\nu^{-1} \rho\right)^{k-1} \rtimes T \\
& \Downarrow \text { (Lemma 2.2) } \\
& \pi \hookrightarrow \mathcal{L}\left(\nu^{-1} \rho, \delta([\rho, \nu \rho])\right) \times \mathcal{L}\left(\delta\left(\left[\nu^{-1} \rho, \rho\right]\right), \delta([\rho, \nu \rho])\right)^{j} \rtimes \lambda
\end{aligned}
$$

for some irreducible $\lambda \leq\left(\nu^{-1} \rho\right)^{k-1} \rtimes T$. It follows from this embedding that $f_{\pi}\left(\nu^{-1} \rho\right) \leq 1+f_{\lambda}\left(\nu^{-1} \rho\right) \Rightarrow f_{\lambda}\left(\nu^{-1} \rho\right) \geq k-1$. By (1.2), this implies $\lambda=$ $L\left(\left(\nu^{-1} \rho\right)^{k-1} ; T\right)$, matching the $\lambda$ in the statement of the proposition. Then,

$$
\begin{aligned}
\pi & \hookrightarrow \mathcal{L}\left(\nu^{-1} \rho, \delta([\rho, \nu \rho])\right) \times \mathcal{L}\left(\delta\left(\left[\nu^{-1} \rho, \rho\right]\right), \delta([\rho, \nu \rho])\right)^{j} \rtimes L\left(\left(\nu^{-1} \rho\right)^{k-1} ; T\right) \\
& \hookrightarrow \mathcal{L}\left(\nu^{-1} \rho, \delta([\rho, \nu \rho])\right) \times \mathcal{L}\left(\delta\left(\left[\nu^{-1} \rho, \rho\right]\right), \delta([\rho, \nu \rho])\right)^{j} \times(\nu \rho)^{f_{\lambda}} \rtimes L\left(\left(\nu^{-1} \rho\right)^{k^{*}} ; T^{*}\right) \\
& \cong(\nu \rho)^{f_{\lambda}} \times \mathcal{L}\left(\nu^{-1} \rho, \delta([\rho, \nu \rho])\right) \times \mathcal{L}\left(\delta\left(\left[\nu^{-1} \rho, \rho\right]\right), \delta([\rho, \nu \rho])\right)^{j} \rtimes L\left(\left(\nu^{-1} \rho\right)^{k^{*}} ; T^{*}\right)
\end{aligned}
$$

by the irreducibility in Lemma 8.1 and Lemma 1.3.3 of [J5]. As $\mathcal{L}\left(\nu^{-1} \rho, \delta([\rho, \nu \rho])\right)$ $\hookrightarrow \nu \rho \times \zeta\left(\left[\nu^{-1} \rho, \rho\right]\right)$, we have

$$
\pi \hookrightarrow(\nu \rho)^{f_{\lambda}+1} \times \zeta\left(\left[\nu^{-1} \rho, \rho\right]\right) \times \mathcal{L}\left(\delta\left(\left[\nu^{-1} \rho, \rho\right]\right), \delta([\rho, \nu \rho])\right)^{j} \rtimes L\left(\left(\nu^{-1} \rho\right)^{k^{*}} ; T^{*}\right) .
$$

Note that it follows immediately from this embedding that $f_{\pi}(\nu \rho)=f_{\lambda}+1$.

To finish, note that by Lemma 8.1, we have

$$
\pi \hookrightarrow(\nu \rho)^{f_{\lambda}+1} \times\left(\nu^{-1} \rho\right)^{k^{*}} \times \zeta\left(\left[\nu^{-1} \rho, \rho\right]\right) \times \mathcal{L}\left(\delta\left(\left[\nu^{-1} \rho, \rho\right]\right), \delta([\rho, \nu \rho])\right)^{j} \rtimes T^{*} .
$$

Reversing the argument used above, we get

$$
\begin{gathered}
\mathcal{L}\left(\delta\left(\left[\nu^{-1} \rho, \rho\right]\right), \delta([\rho, \nu \rho])\right)^{j} \rtimes T^{*} \hookrightarrow \delta\left(\left[\nu^{-1} \rho, \rho\right]\right)^{2 j} \rtimes T^{*} \\
\Downarrow \\
\pi \hookrightarrow(\nu \rho)^{f_{\lambda}+1} \times\left(\nu^{-1} \rho\right)^{k^{*}} \times \zeta\left(\left[\nu^{-1} \rho, \rho\right]\right) \times \delta\left(\left[\nu^{-1} \rho, \rho\right]\right)^{2 j} \rtimes T^{*} .
\end{gathered}
$$

Finally, as $\zeta\left(\left[\nu^{-1} \rho, \rho\right]\right) \hookrightarrow \nu^{-1} \rho \times \rho$, we have

$$
\begin{aligned}
\pi & \hookrightarrow(\nu \rho)^{f_{\lambda}+1} \times\left(\nu^{-1} \rho\right)^{k^{*}} \times \nu^{-1} \rho \times \rho \times \delta\left(\left[\nu^{-1} \rho, \rho\right]\right)^{2 j} \rtimes T^{*} \\
& \cong(\nu \rho)^{f_{\lambda}+1} \times\left(\nu^{-1} \rho\right)^{k^{*}+1} \times \delta\left(\left[\nu^{-1} \rho, \rho\right]\right)^{2 j} \rtimes\left(\rho \rtimes T^{*}\right) .
\end{aligned}
$$

The result now follows from Lemma 2.4.

The case where $\ell$ is even is similar. 
Proposition 8.6. Let $\pi=L\left(\left(\nu^{\frac{-a+1}{2}}\right)^{k}, \delta\left(\left[\nu^{\frac{-a+1}{2}} \rho, \nu^{\frac{a-3}{2}} \rho\right]\right)^{\ell} ; T\right)$ with $a>3$, a odd (and $\left.\operatorname{red}(\rho ; \sigma) \equiv \frac{1}{2} \bmod 1\right)$. Suppose $f_{\pi}\left(\nu^{\frac{a-3}{2}} \rho\right)=0$. Then we must have $\ell=0$ and $(\rho, a-2) \notin \operatorname{Jord}(T)$. If $\mu_{\left\{\nu \frac{a-1}{2}\right.}^{*}(T)=\left(\nu^{\frac{a-1}{2}} \rho\right)^{f_{T}} \otimes T^{\prime}$ (Theorem 3.1), then

$$
\mu_{\left\{\nu \frac{a-1}{2} \rho\right\}}^{*}(\pi)=\left(\nu^{\frac{a-1}{2}} \rho\right)^{k+f_{T}} \otimes T^{\prime} .
$$

Proof. First, the same argument as in the proof of Proposition 3.4 tells us that to have $f_{\pi}\left(\nu^{\frac{a-3}{2}} \rho\right)=0$, we must have $\ell=0$ and $f_{T}\left(\nu^{\frac{a-3}{2}} \rho\right)=0$. However, in this case, $f_{T}\left(\nu^{\frac{a-3}{2}} \rho\right)=0$ requires $(\rho, a-2) \notin \operatorname{Jord}(T)$ (Theorem 3.1). By Note 6.2. $\nu^{\frac{-a+1}{2}} \rho \rtimes T$ is irreducible. Therefore, noting that $\nu^{\frac{-a+1}{2}} \rho \times \nu^{\frac{a-1}{2}} \rho$ is irreducible (as $a>3)$,

as claimed.

$$
\begin{aligned}
& \pi \hookrightarrow\left(\nu^{\frac{-a+1}{2}} \rho\right)^{k} \rtimes T \\
& \cong\left(\nu^{\frac{-a+1}{2}} \rho\right)^{k-1} \times \nu^{\frac{a-1}{2}} \rho \rtimes T \\
& \cong\left(\nu^{\frac{a-1}{2}} \rho\right) \times\left(\nu^{\frac{-a+1}{2}} \rho\right)^{k-1} \rtimes T \\
& \vdots \\
& \cong\left(\nu^{\frac{a-1}{2}} \rho\right)^{k} \rtimes T \\
& \cong\left(\nu^{\frac{a-1}{2}} \rho\right)^{k+f_{T} \rtimes T^{\prime}} \\
& \Downarrow \\
& \mu_{\left\{\nu^{\frac{a-1}{2}} \rho\right\}}^{*}(\pi)=\left(\nu^{\frac{-a+1}{2}} \rho\right)^{k+f_{T}} \otimes T^{\prime},
\end{aligned}
$$

\section{ACKNOWLEDGMENTS}

We take this opportunity to thank both the referee and Ivan Matić for their help in improving the paper.

\section{REFERENCES}

[Alv] Dean Alvis, The duality operation in the character ring of a finite Chevalley group, Bull. Amer. Math. Soc. (N.S.) 1 (1979), no. 6, 907-911, DOI 10.1090/S0273-0979-1979-14690-1. MR.546315

[Art] James Arthur, The endoscopic classification of representations, American Mathematical Society Colloquium Publications, vol. 61, American Mathematical Society, Providence, RI, 2013. Orthogonal and symplectic groups. MR 3135650

[Au] Anne-Marie Aubert, Dualité dans le groupe de Grothendieck de la catégorie des représentations lisses de longueur finie d'un groupe réductif p-adique (French, with English summary), Trans. Amer. Math. Soc. 347 (1995), no. 6, 2179-2189, DOI 10.2307/2154931. MR 1285969

[Ba] Dubravka Ban, Parabolic induction and Jacquet modules of representations of $\mathrm{O}(2 n, F)$, Glas. Mat. Ser. III 34(54) (1999), no. 2, 147-185. MR1739616

[B-J1] Dubravka Ban and Chris Jantzen, Degenerate principal series for even-orthogonal groups, Represent. Theory 7 (2003), 440-480, DOI 10.1090/S1088-4165-03-00166-3. MR2017065

[B-J2] Dubravka Ban and Chris Jantzen, Duality and the normalization of standard intertwining operators, Manuscripta Math. 115 (2004), no. 4, 401-415, DOI 10.1007/s00229-004-05047. MR2103658

[B-J3] Dubravka Ban and Chris Jantzen, Jacquet modules and the Langlands classification, Michigan Math. J. 56 (2008), no. 3, 637-653, DOI 10.1307/mmj/1231770365. MR2490651

[B-M] Dan Barbasch and Allen Moy, A unitarity criterion for p-adic groups, Invent. Math. 98 (1989), no. 1, 19-37, DOI 10.1007/BF01388842. MR.1010153

[B-Z] I. N. Bernstein and A. V. Zelevinsky, Induced representations of reductive $\mathfrak{p}$-adic groups. I, Ann. Sci. École Norm. Sup. (4) 10 (1977), no. 4, 441-472. MR0579172 
[B-W] Armand Borel and Nolan R. Wallach, Continuous cohomology, discrete subgroups, and representations of reductive groups, Annals of Mathematics Studies, vol. 94, Princeton University Press, Princeton, N.J.; University of Tokyo Press, Tokyo, 1980. MR.554917

[Ca] W. Casselman, Introduction to the theory of admissible representations of p-adic reductive groups, preprint (available online at www.math.ubc.ca/people/faculty/cass/research.html as "The $p$-adic notes").

[Cur] Charles W. Curtis, Truncation and duality in the character ring of a finite group of Lie type, J. Algebra 62 (1980), no. 2, 320-332, DOI 10.1016/0021-8693(80)90185-4. MR563231

[D-L1] Pierre Deligne and George Lusztig, Duality for representations of a reductive group over a finite field, J. Algebra 74 (1982), no. 1, 284-291, DOI 10.1016/0021-8693(82)90023-0. MR644236

[D-L2] Pierre Deligne and George Lusztig, Duality for representations of a reductive group over a finite field. II, J. Algebra 81 (1983), no. 2, 540-545, DOI 10.1016/0021-8693(83)90202-8. MR700298

[G-L] W.-T. Gan and L. Lomelí, Globalization of supercuspidal representations over function fields and applications, to appear, J. Eur. Math. Soc.

[I-M] N. Iwahori and H. Matsumoto, On some Bruhat decomposition and the structure of the Hecke rings of $\mathfrak{p}$-adic Chevalley groups, Inst. Hautes Études Sci. Publ. Math. 25 (1965), 5-48. MR0185016

[J1] Chris Jantzen, Reducibility of certain representations for symplectic and odd-orthogonal groups, Compositio Math. 104 (1996), no. 1, 55-63. MR 1420710

[J2] Chris Jantzen, On supports of induced representations for symplectic and odd-orthogonal groups, Amer. J. Math. 119 (1997), no. 6, 1213-1262. MR1481814

[J3] Chris Jantzen, Some remarks on degenerate principal series, Pacific J. Math. 186 (1998), no. 1, 67-87, DOI 10.2140/pjm.1998.186.67. MR1665057

[J4] Chris Jantzen, Duality and supports of induced representations for orthogonal groups, Canad. J. Math. 57 (2005), no. 1, 159-179, DOI 10.4153/CJM-2005-007-x. MR2113853

[J5] Chris Jantzen, Jacquet modules of p-adic general linear groups, Represent. Theory 11 (2007), 45-83, DOI 10.1090/S1088-4165-07-00316-0. MR2306606

[J6] Chris Jantzen, Discrete series for $p$-adic $\mathrm{SO}(2 n)$ and restrictions of representations of $\mathrm{O}(2 n)$, Canad. J. Math. 63 (2011), no. 2, 327-380, DOI 10.4153/CJM-2011-003-2. MR2809059

[J7] Chris Jantzen, Tempered representations for classical p-adic groups, Manuscripta Math. 145 (2014), no. 3-4, 319-387, DOI 10.1007/s00229-014-0679-5. MR3268853

[J8] Chris Jantzen, Jacquet modules and irrreducibility of induced representations for classical p-adic groups, Manuscripta Math. 156 (2018), no. 1-2, 23-55, DOI 10.1007/s00229-0170955-2. MR.3783564

[Kat] Shin-ichi Kato, Duality for representations of a Hecke algebra, Proc. Amer. Math. Soc. 119 (1993), no. 3, 941-946, DOI 10.2307/2160536. MR 1215028

[Kaw] N. Kawanaka, Fourier transforms of nilpotently supported invariant functions on a simple Lie algebra over a finite field, Invent. Math. 69 (1982), no. 3, 411-435, DOI 10.1007/BF01389363. MR679766

[K-Z] Harold Knight and Andrei Zelevinsky, Representations of quivers of type $A$ and the multisegment duality, Adv. Math. 117 (1996), no. 2, 273-293, DOI 10.1006/aima.1996.0013. MR 1371654

[Kon] Takuya Konno, A note on the Langlands classification and irreducibility of induced representations of p-adic groups, Kyushu J. Math. 57 (2003), no. 2, 383-409, DOI 10.2206/kyushujm.57.383. MR.2050093

[Mœ1] C. Mœglin, Normalisation des opérateurs d'entrelacement et réductibilité des induites des cuspidales; le cas des groupes classiques p-adiques, Ann. of Math., 151(2000), 817-847.

[Mœ2] C. Mœglin, Sur la classification des séries discrètes des groupes classiques p-adiques: paramètres de Langlands et exhaustivité (French, with English summary), J. Eur. Math. Soc. (JEMS) 4 (2002), no. 2, 143-200, DOI 10.1007/s100970100033. MR1913095

[Mœ3] C. Mœglin, Sur certains paquets d'Arthur et involution d'Aubert-Schneider-Stuhler généralisée (French, with English summary), Represent. Theory 10 (2006), 86-129, DOI 10.1090/S1088-4165-06-00270-6. MR2209850 
[Mœ4] Colette Mœglin, Paquets stables des séries discrètes accessibles par endoscopie tordue; leur paramètre de Langlands (French, with English summary), Automorphic forms and related geometry: assessing the legacy of I. I. Piatetski-Shapiro, Contemp. Math., vol. 614, Amer. Math. Soc., Providence, RI, 2014, pp. 295-336, DOI 10.1090/conm/614/12254. MR3220932

[M-T] Colette Mœglin and Marko Tadić, Construction of discrete series for classical p-adic groups, J. Amer. Math. Soc. 15 (2002), no. 3, 715-786, DOI 10.1090/S0894-0347-0200389-2. MR 1896238

[M-W] C. Mœglin and J.-L. Waldspurger, Sur l'involution de Zelevinski (French), J. Reine Angew. Math. 372 (1986), 136-177, DOI 10.1515/crll.1986.372.136. MR863522

[Mu1] Goran Muić, The unitary dual of p-adic $G_{2}$, Duke Math. J. 90 (1997), no. 3, 465-493, DOI 10.1215/S0012-7094-97-09012-8. MR.1480543

[Mu2] Goran Muić, Composition series of generalized principal series; the case of strongly positive discrete series, Israel J. Math. 140 (2004), 157-202, DOI 10.1007/BF02786631. $\operatorname{MR} 2054843$

[Mu3] Goran Muić, Reducibility of generalized principal series, Canad. J. Math. 57 (2005), no. 3, 616-647, DOI 10.4153/CJM-2005-025-4. MR2134404

$[\mathrm{Mu}-\mathrm{T}]$ Goran Muić and Marko Tadić, Unramified unitary duals for split classical p-adic groups; the topology and isolated representations, On certain $L$-functions, Clay Math. Proc., vol. 13, Amer. Math. Soc., Providence, RI, 2011, pp. 375-438. MR2767523

[S-S] Peter Schneider and Ulrich Stuhler, Representation theory and sheaves on the Bruhat-Tits building, Inst. Hautes Études Sci. Publ. Math. 85 (1997), 97-191. MR1471867

[Sh1] Freydoon Shahidi, A proof of Langlands' conjecture on Plancherel measures; complementary series for p-adic groups, Ann. of Math. (2) 132 (1990), no. 2, 273-330, DOI 10.2307/1971524. MR 1070599

[Sh2] Freydoon Shahidi, Twisted endoscopy and reducibility of induced representations for $p$ adic groups, Duke Math. J. 66 (1992), no. 1, 1-41, DOI 10.1215/S0012-7094-92-06601-4. MR 1159430

[Si1] Allan J. Silberger, The Langlands quotient theorem for p-adic groups, Math. Ann. 236 (1978), no. 2, 95-104, DOI 10.1007/BF01351383. MR0507262

[Si2] Allan J. Silberger, Special representations of reductive p-adic groups are not integrable, Ann. of Math. (2) 111 (1980), no. 3, 571-587, DOI 10.2307/1971110. MR577138

[T1] Marko Tadić, Structure arising from induction and Jacquet modules of representations of classical p-adic groups, J. Algebra 177 (1995), no. 1, 1-33, DOI 10.1006/jabr.1995.1284. MR.1356358

[T2] Marko Tadić, On reducibility of parabolic induction, Israel J. Math. 107 (1998), 29-91, DOI 10.1007/BF02764004. MR1658535

[T3] Marko Tadić, On classification of some classes of irreducible representations of classical groups, Representations of real and p-adic groups, Lect. Notes Ser. Inst. Math. Sci. Natl. Univ. Singap., vol. 2, Singapore Univ. Press, Singapore, 2004, pp. 95-162, DOI 10.1142/9789812562500_0004. MR2090870

[T4] Marko Tadić, On invariants of discrete series representations of classical p-adic groups, Manuscripta Math. 135 (2011), no. 3-4, 417-435, DOI 10.1007/s00229-010-0423-8. $\operatorname{MR} 2813443$

[T5] Marko Tadić, On tempered and square integrable representations of classical p-adic groups, Sci. China Math. 56 (2013), no. 11, 2273-2313, DOI 10.1007/s11425-013-46670. MR3123571

[Wa] J.-L. Waldspurger, La formule de Plancherel pour les groupes p-adiques (d'après HarishChandra) (French, with French summary), J. Inst. Math. Jussieu 2 (2003), no. 2, 235-333, DOI 10.1017/S1474748003000082. MR.1989693

[Ze] A. V. Zelevinsky, Induced representations of reductive $\mathfrak{p}$-adic groups. II. On irreducible representations of GL( $n)$, Ann. Sci. École Norm. Sup. (4) 13 (1980), no. 2, 165-210. MR584084

[Zh] Yuanli Zhang, L-packets and reducibilities, J. Reine Angew. Math. 510 (1999), 83-102, DOI 10.1515/crll.1999.050. MR.1696092 
Department of Mathematics, East Caronlina University, Greenville, North CarOLINA 27858

Email address: jantzenc@ecu.edu 